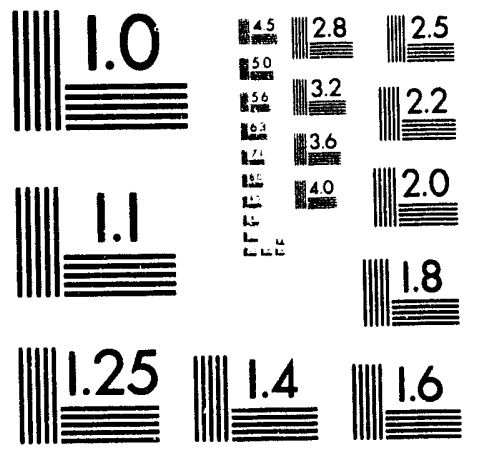



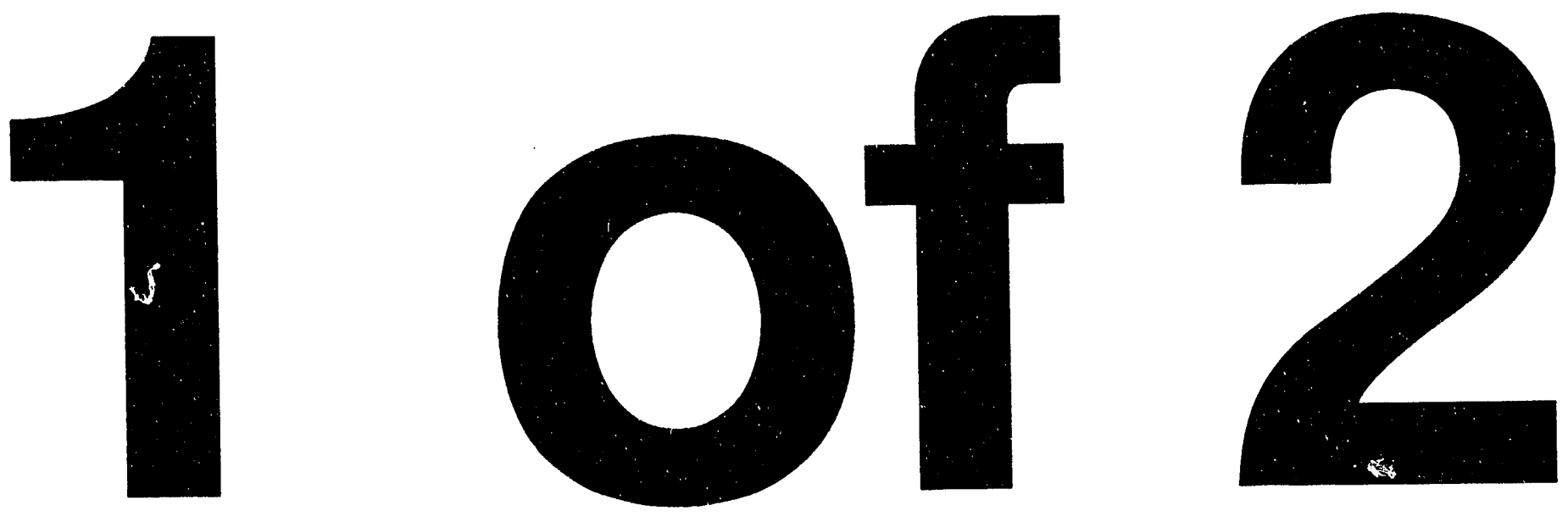
SAND93-0256

Unlimited Release

Printed March 1993
Distribution

Category UC-403

\title{
EVENT TRIGGERED DATA ACQUISITION IN THE ROCK MECHANICS LABORATORY
}

\author{
Robert D. Hardy \\ Geomechanics Department \\ Sandia National Laboratories \\ Albuquerque, New Mexico 87185
}




\section{Acknowledgements}

DATAVG is based on a program written by D. J. Holcomb in 1983.

I wish to thank S. R. Brown and D. J. Holcomb for their suggestions while writing the code. I also wish to thank S. R. Brown and W. R. Wawersik for their review of the manuscript and their helpful suggestions. 


\section{Contents}

1 BACKGROUND 9

2 OVERVIEW and DEFINITIONS $\quad \theta$

3 OPERATION 10

3.1 Set Up Channels . . . . . . . . . . . . . . . . . . 10

3.1 .1 Test Identification $\ldots \ldots \ldots \ldots$

3.1.2 Channel Selection ................. 11

3.1.3 Channel Name . . . . . . . . . . . . . . . 11

3.1.4 Channel Scale Factor . . . . . . . . . . . . . 11

$3.1 .5 \quad$ Trigger Value . . . . . . . . . . . . . . . 11

3.1.6 Data Offset Removal . . . . . . . . . . . . . . 11

3.1.7 Interface Hardware for this Channel . . . . . . . . . . 11

3.1.8 Completion of Channel Set Up . . . . . . . . . . . 12

$3.2 \quad$ Set U Screen . . . . . . . . . . . . . . . . . 12

$3.2 .1 \quad$ Number of Windows . . . . . . . . . . . 12

3.2 .2 Which Window . . . . . . . . . . . . . 12

3.3 Print Channel Data .................... 13

3.4 Check Channel Set Up . . . . . . . . . . . . . . 13

$3.5 \quad$ Save Setup Data . . . . . . . . . . . . . . . 13

$3.6 \quad$ Name Data File . . . . . . . . . . . . . . . . . . . 14

3.7 Gather Data . . . . . . . . . . . . . . . . . . 14

3.7.1 Force Recording of a Sample (F1) . . . . . . . . . 15

$3.7 .2 \quad$ Reset a Window $(\mathrm{F} 2) \ldots \ldots \ldots \ldots$

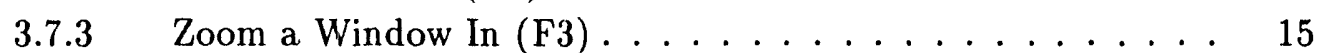

3.7.4 Zoom a Window Ou.t (F4) . . . . . . . . . . . . 15

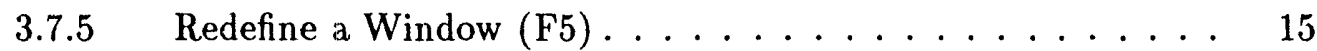

3.7.6 Clear a Window $(\mathrm{F} 6) \ldots \ldots \ldots \ldots$

3.7 .7 Clear all Windows $(\mathrm{F} 7) \ldots \ldots \ldots \ldots$

3.7 .8 Start a new Data File (F8) . . . . . . . . . . . 16

3.7 .9 End the Test (F9) $\ldots \ldots \ldots \ldots \ldots \ldots$

$3.8 \quad$ Recall Setup Data . . . . . . . . . . . . . . 16

3.9 Exit to DOS ..................... 16

4 TEMPERATURE CHANNELS

5 DISK FILES

$5.1 \quad$ Setup File . . . . . . . . . . . . . . . 17

$5.2 \quad$ Header File . . . . . . . . . . . . . . . . . 18

$5.3 \quad$ Data File . . . . . . . . . . . . . . . . . . . . . . . . . . . . 19

5.4 Thermocouple Data Tables . . . . . . . . . . . . . 19 
6 INSTALLATION NOTES 19

6.1 Keithley Box Configuration File . . . . . . . . . . . . . . 20

6.2 Temperature Data Files . . . . . . . . . . . . 20

7 LIMITATIONS AND REQUIREMENTS 21

7.1 File Management . . . . . . . . . . . . . . . . . . . . 21

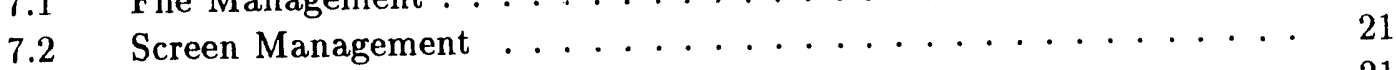

7.3 Time Interval Triggering Limits . . . . . . . . . . . . . . 21

8 COMPUTED CHANNEL DESIGN 21

8.1 COMPUTED CHANNEL INTERFACE . . . . . . . . . . 21

8.1.1 Computed Channel Driver Function Prototype . . . . . . 22

8.1 .2 Initialize Computed Channel . . . . . . . . . . . 22

8.1.3 Save Computed Channel Set Up Data . . . . . . . . . . . 23

8.1.4 Restore Computed Channel Configuration . . . . . . . . . 23

8.1.5 Normal Computed Channel Operation . . . . . . . . . 23

8.1 .6 Support Functions . . . . . . . . . . . . . . 23

8.1.6.1 Get an Integer with prompt and default. . . . . . . . 23

8.1.6.2 Get a Float with prompt and default. . . . . . . . . 23

8.1.6.3 Get a Board and Channel. . . . . . . . . . . . . . . . 24

8.1.6.4 Validate a File Name. . . . . . . . . . . . . . . 24

9 PROGRAM STRUCTURE

10 VARIABLES 25

10.1 Global General Data . . . . . . . . . . . . . . . . . . 25

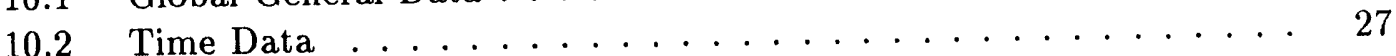

10.3 Plot Data . . . . . . . . . . . . . . . . . . . . 27

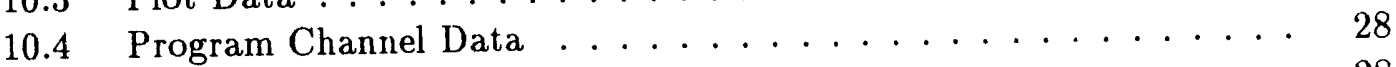

10.5 Hardware Information . . . . . . . . . . . . . . . . . . . 28

10.6 Other Variables . . . . . . . . . . . . . . . . 29

A DATAVG.H

B DATAVG.C

B .1 TEMP.C - Temperature Channel Driver . . . . . . . . . . . 73

C 500LIB.H

D 500LIB.C

$\begin{array}{ll}\text { E SRBGRAPH.H } & 01\end{array}$

F SRBGRAPH Composite Source 93

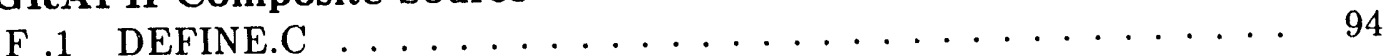

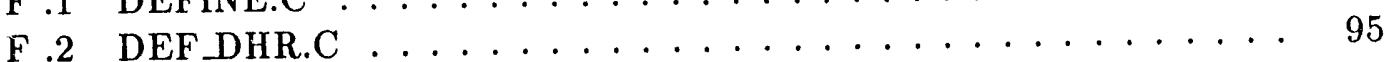

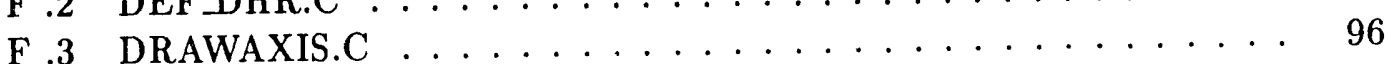

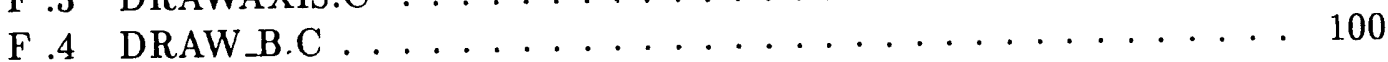




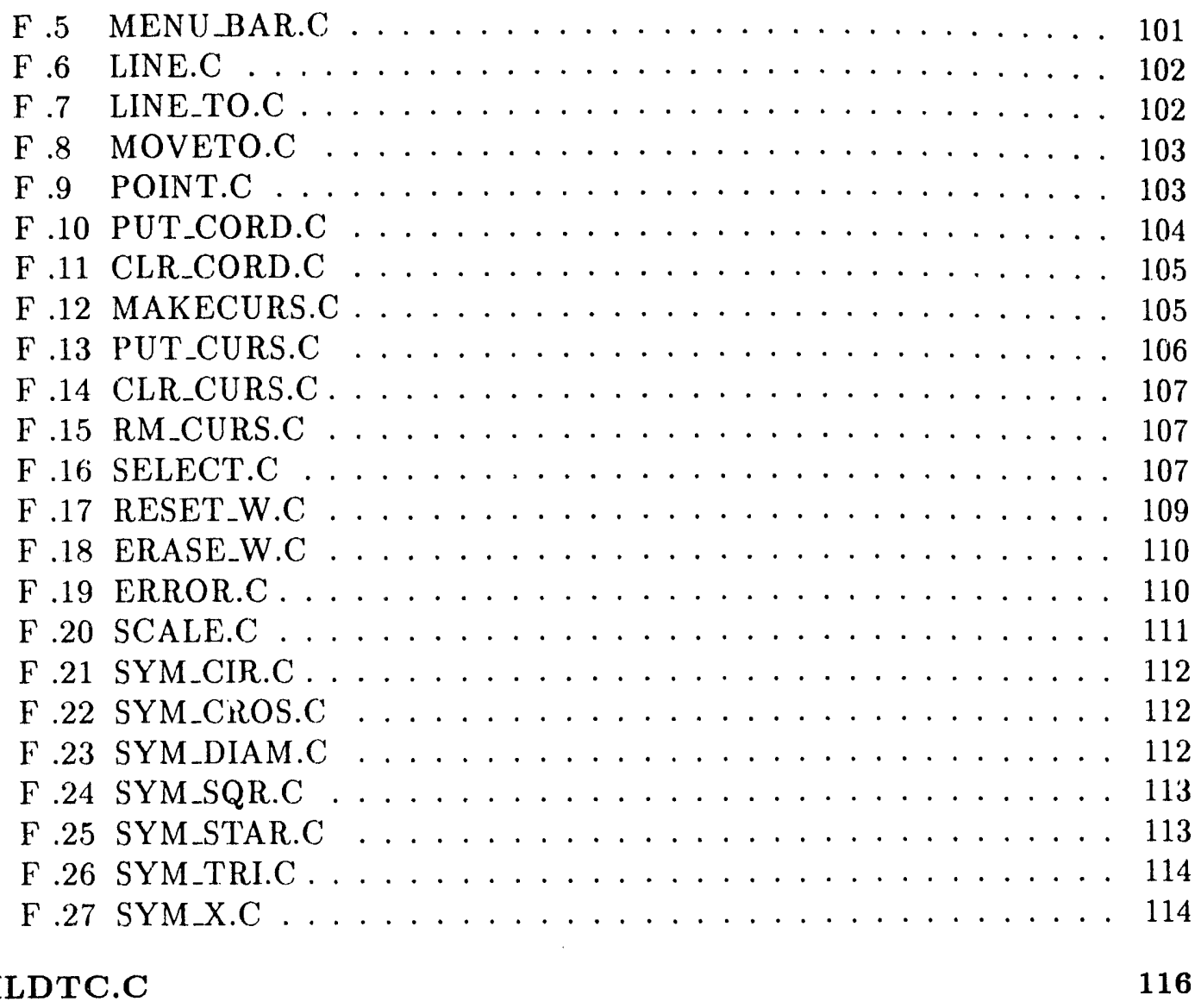

$\begin{array}{ll}\text { G BUILDTC.C } & 116\end{array}$

\section{List of Figures}

Setup File . . . . . . . . . . . . . . . . . 17

Header File . . . . . . . . . . . . . . . . . . . 18

Data File . . . . . . . . . . . . . . . . . . . . 19

Keithley Configuration File, K500.CFG . . . . . . . . . . . 20 


\section{BACKGROUND}

Increasing complexity of experiments coupled with limitations of the previously used computers required improvements in both hardware and software in the Rock Mechanics Laboratories. Increasing numbers of input channels and the need for better graphics could no longer be supplied by DATAVG, an existing software package for data acquisition and display written by D. J. Holcomb in 1983. After researching the market and trying several alternatives, no commercial program was found which met our needs. The previous version of DATAVG had the basic features needed but was tied to obsolete hardware. Memory limitations on the previously used PDP-11 made it impractical to upgrade the software further. With the advances in IBM compatible computers it is now desirable to use them as data recording platforms.

With this information in mind, it was decided to write a new version of DATAVG which would take advantage of newer hardware. The new version had to support multiple graphic display windows and increased channel counts. It also had to be easier to use.

\section{OVERVIEW and DEFINITIONS}

This document describes a new and greatly expanded version of DATAVG as used to record data in the Rock Mechanics Laboratory at Sandia National Laboratories/New Mexico. DATAVG is currently at Version 1.10.

DATAVG is an event triggered data logging prograrn. An event is defined as a change in the quantity measured at any input channel which exceeds a user defined threshold. The triggering threshold, DELTA, is the absolute value of the data change since the last recorded reading. Input channels are continuously scanned. When a specified change in value has been met at any channel, the data from all channels is recorded in a file and plotted on the video screen.

Allowing any input to trigger recording facilitates tests in which there are several phases. During each phase of the test a different set of input parameters may be of interest. With DATAVG, data recording may proceed without user intervention to change sampling rates, etc.

Event triggering also minimizes the amount of data recorded. With many commercial programs sampling is at uniform time intervals. If a test is to run for a long time but some part of the test requires fast sampling, as near failure of a specimen, a lange amount of nearly useless data is recorded. DATAVG avoids this excess data by triggeri: $;$ at long time intervals but short intervals in some other variable.

For instance, the load on a sample may be varied in a series of steps. At each step some process is to be performed. With DATAVG the changes in load will trigger recording during the load change phase. During the other process the load will be constant but some other variable will trigger recording. If nothing else is changing, the time channel will cause some minimal recording rate. See section 7.3 for further information on triggering at time intervals.

DATAVG supports one to four windows for data plotting and emulates an X-Y plotter. Strip chart style plots are not supported. Each window displays data from two channels. The same channel may be displayed in more than one window if needed. 


\section{OPERATION}

DATAVG is menu-driven with the menus arranged in approximately the order required to operate the program as shown below.

1) SET UP CHANNELS

2) SET UP SCREEN

3) PRINT CHANNEL DATA

4) CHECK CHANNEL SETUP

5) SAVE SETUP DATA

6) NAME DATA FILE

7) GATHER DATA

8) RECALL SETUP DATA

9) EXIT TO DOS

Item three, Print Channel Data, is for user convenience and compatibility with the old version. It is convenient for test documentation. Item six, Name Data File, was carried over from the previous version. The Gather Data operation, item seven, will request the file name if it was not defined by item six.

If the setup has been saved in a disk file it may be recalled by item eight. This will eliminate any need for additional setup if a test is to be repeated. The channel and screen data are saved in a form which allows operation immediately after recall. By use of this feature a repeat test may be run by selecting menu item eight followed by menu item seven.

Saved setup files may be recalled then edited by using menu items one or two. It is possible to edit the screen display during a test then save the changes by selecting menu item five before leaving DATAVG. If saving a setup after the data recording runs, first check the First Point Zero flag by using the Channel Setup menu operation. If the first reading happened to be zero, this flag would be altered.

Menu items six and seven ask for a file name to be used for data storage. Two file names are created by appending extensions to the name supplied by the user. The first is "filename.HDR" which stores the program version, a copy of the setup information, a time stamp, column headings and the offsets which are subtracted from subsequent readings. The second file is "filename.DAT" which contains all data for the test. The ".DAT" file is closed and reopened at selected intervals for data security reasons.

Menu operations will now be discussed in order.

\subsection{Set Up Channels}

In the channel set up routine, most requested values show a default in parentheses immediately before the prompt "?". If this value is satisfactory it may be selected by a carriage return alone.

\subsubsection{Test Identification}

The first question asked is the test identification. The current test identification is shown and may be changed by entering a new identification. If the current identification is correct, as when editing a set up, just press ENTER. 


\subsubsection{Channel Selection}

Channel set up requests information on the data to be recorded in logical channel order starting with the channel next higher than the highest one previously selected. Channels may be defined in any order by entering the number of the desired channel.

Channel zero $(0)$ is always TIME. TIME is always recorded in seconds starting at the beginning of the test. A prompt will ask for the maximum interval between recordings. This interval is used to trigger recording if other channels are changing slowly.

\subsubsection{Channel Name}

The user is asked for the name to be used with this channel. The currently assigned name is the default which may be retained by pressing ENTER. The channel name is used for the column heading in the data file and axis labels on plots. Measurement units may be included in the channel name, for example:

LOAD (Kn) or STROKE(in.).

\subsubsection{Channel Scale Factor}

The next prompt is for units per Volt. This is the real quantity corresponding to +1 volt input. For example, a load cell conditioner provides $5 \mathrm{~V}$ at $25000 \mathrm{lbs}$. tensile load. When DATAVG asks for the units per Volt value respond with $5000 \mathrm{lbs}$. (or 5 KIPS where $1 \mathrm{KIP}$ $=1000 \mathrm{lbs}$ ). If compression is to be positive load, enter $-5000 \mathrm{lbs}$. or $-5 \mathrm{KIPS}$. The units are not entered but are shown here for clarity. With this information DATAVG can scale readings into proper engineering units.

The calibration plotting software used in the Rock Mechanics Lab provides units per Volt in the graph header.

\subsubsection{Trigger Value}

The third prompt asks for the input change, in real units, required before recording data is initiated. While recording data, DATAVG continuously scans all input channels. The current readings are compared to the last readings in the data file. When the change equals or exceeds the value entered here the current readings will be recorded in the file and all plots are updated.

\subsubsection{Data Offset Removal}

The fourth prompt asks if the first data point is to be used as the zero offset. Frequently transducer outputs are not exactly zero at the start of a test. If you are only interested in the change during a test answer this question by pressing ENTER. If true readings are required, answer by pressing " $\mathrm{N}$ ". Data offsetting is done by subtracting the first readings of a test from all subsequent readings. The offset values are recorded in the data header file so true readings may be restored if this is later required.

\subsubsection{Interface Hardware for this Channel}

Next the hardware to be used is specified. The user is asked for the interface board in the Keithley box and the channel to be used. The prompt includes a menu of available 
boards. Select the board by number from the menu. The menu item numbers correspond to the slot numbers in the Keithley 500 box. Item numbers higher than ten are computed channel pseudo boards. Entries with no corresponding board name will be rejected. After selecting a board, the user is asked for the channel on that board to be used. This prompt includes the valid range of channel numbers and only valid channels will be accepted. The default is the channel following the last one selected on this board.

There is a possibility for confusion in selecting hardware channel numbers. The interface boards use a zero based numbering system. Each board starts numbering at zero. DATAVG uses logical channels starting at zero also, but they do not start over on each board. The user must plan hardware usage and be careful to enter proper data.

If the "TEMP" board is selected, a prompt will ask for the thermocouple type. This allows an appropriate conversion to be applied to get degrees Celsius from each reading taken. If there is no AIM7 board or if TIIERMO.CFG is missing, temperature readings may not be made.

\subsubsection{Completion of Channel Set Up}

After answering all questions about a channel, DATAVG returns to step 3.1.2. After all channels have been defined, enter "DONE" for the channel number.

Channel setup information will be displayed on the screen in tabular form. Channel zero, if defined, will show a units/Volt value of 1 . This is normal and will in no way limit the recording time for a test. It only reflects a scale factor of one which is ajplied to time readings.

Press enter to return to the main menu.

\subsection{Set Up Screen}

\subsubsection{Number of Windows}

DATAVG will display the number of windows currently defined then ask for the number of windows needed. The options are one through four. Invalid responses will be rejected.

During initial setup there are no windows defined an.t most defaults will be zero. The default $X$ axis channel will be -1 indicating an urused window.

\subsubsection{Which Window}

After selecting the number of windows, a prompt will ask which window to set up. The default window will start at one and step to the next higher numbered window each time through. Any valid window may be selected instead of the default by entering it's number. Information about the data to be plotted in that window will be requested. This operation repeats until all windows have been set up. If a window is selected which is already configured properly, just take the default values at each question.

First, the user is asked for the number of the channel to be plotted on the $\mathrm{X}$ axis then the number of the channel to be plotted on the $Y$ axis. The defaults are the currently defined channels. The first time a window is set up the $X$ axis default will be -1 and the $\mathrm{Y}$ axis channel will be zero. 
If -1 is entered for the $X$ axis channel, this window will be blank when data recording starts. Blank windows may be activated during a test by redefining them with a real channel on the $\mathrm{X}$ axis.

Next, the user will be asked in turn for the minimum and maximum data values to plot on each axis. Default values will be displayed and they may be accepted by pressing ENTER. During initiai setup the defaults will be zero. The minimum value plotted must be smaller than the maximum. If the maximum value entered is smaller than the minimum value, DATAVG will exchange them so the plot routine will be happy.

After configuring the last window, a prompt will ask if all windows are properly configured. Press $Y$ to end, or any other key to go back and redo one or more windows. This function may be terninated at any time by entering -1 for the window number.

\subsection{Print Channel Data}

Selecting this option prints a table of information about this setup. Data is shown in channel number order with only active channels displayed. The table shows the number and name of each channel. This is followed by the full scale value and trigger threshold (DELTA). Last is a zero offset column. If the zero offset value is non-zero, the first reading will be subtracted from all recorded data. Usually the zero offset is one or zero, but it may have any value.

Channel zero, if defined, will show a full scale value of 10 . This is normal and will in no way limit the recording time for a test. It only reflects a scale factor of one which is applied to time readings.

Output will be sent to PRN: which may be redirected as needed. After printing the chart, the main menu will be displayed.

\subsection{Check Channel Set Up}

This operation steps through the channels starting with channel one and displays readings in volts. No scaling is performed. This allows confirmation of readings with a voltmeter. After the reading is shown, the user is asked to change something affecting the channel then press return. A new value will be displayed for confirmation of the change. Pressing return will step to the next channel.

If the letter " $A$ " is entered before the return, the same channel will be displayed again. A channel may be tested as often as needed before going to the next channel.

After all channels have been tested, DATAVG will return to the main menu.

\subsection{Save Setup Data}

This operation saves all setup information in a file. The configuration of all channels and windows is saved in an ASCII file. A name must be provided for the setup file. An existing file of the same name will be replaced. There is no check for or warning before replacing an old file.

After creating the setup file the main menu will be displayed. 


\subsection{Name Data File}

As stated above, this operation is not required. It was retained from the previous version of DATAVG because some users like to see a specific way to name their data file. If this operation is not done explicitly before beginning a test, it will be done automatically.

This operation asks for a file name to be used for data storage. Blank lines are ignored until a name is entered. After a name has been entered it is scanned for characters DOS does not like. These are removed so the file name will be valid. If a complete path is specified, the directory must exist. DATAVG will not create directories. Since DATAVG supplies the file type fields, only characters preceding the dot are kept.

After entering a file name, a prompt asks for the number of data points to be stored before closing and reopening the file. A positive number must be entered. Entering one will force closing of the data file after every point is recorded.

If the test will run unattended for long periods, a small number might be used since data probably will not be recorded very fast. Larger numbers of points per file will increase speed if data will be recorded quickly.

After answering these questions, DATAVG will return to the main menu.

\subsection{Gather Data}

This operation starts data recording. If a data file name has not been specified, this information will be requested as described in Section 3.6.

The first operation is to open the data header file using the name requested above. The header file name is made by appending ".HDR" to the base file name. This header file is described in Section 5.2. The file is opened in append mode to eliminate loss of any previous file of the same name.

Next, all channels will be read and the zero offsets stored in the header file. These data are, also, subtracted from readings taken during the test to remove initial offsets.

The header file is then closed and a new file is opened with the same base name but the type field will be ".DA' $\Gamma$ ". The data file is described in Section 5.3. This file is also opened in append mode and will start with the first data set.

Finally the screen plots are set up. All plots are initialized to display the data ranges specified in Section 3.2. If a window includes a data value of $(0,0)$ in its active area there will be a small cross drawn to mark that location. The cross may be partial if one axis is near zero.

There is a menu across the bottom of the screen. This menu describes the function key actions. If the screen is EGA or better, the menu is two lines with the function key name above it's action. On CGA screens only the function key actions are displayed.

At this time, the program enters a loop. Data is read and compared to the threshold values. If any channel value has crossed the threshold, data from all channels will be recorded and plotted. This loop is repeated until an active function key, as described below, is pressed.

During data recording, there are nine function keys active. Six of them affect windows, and the rest perform various other tasks. Five of the function keys which affect windows perform their task immediately if only one window is active. When multiple windows are active, the user is prompted for the window number. 


\subsubsection{Force Recording of a Sample (F1)}

This function forces recording of a data point. The current reading is stored regardless of threshold crossing.

NOTE:

The following operations are useful but they do cause data logging to stop until the operation is complete. It is recommended that the user be careful in selecting appropriate times to perform these operations to minimize the loss of data.

\subsubsection{Reset a Window (F2)}

This function resets a window to show the maximum data range on both axes. This is useful if the active plot region cannot be seen. If only one window is defined this function is immediate.

\subsubsection{Zoom a Window In (F3)}

This function scales the selected window to show a smaller area and centers the plot on the last data point. Both axes are scaled to $75 \%$ of their previous length. If only one window is defined this function is immediate.

\subsubsection{Zoom a Window Out (F4)}

This function scales the selected window to show a larger area and centers the plot on the last data point. Both axes are scaled to $133 \%$ of their previous length. If only one window is defined this function is immediate.

\subsubsection{Redefine a Window (F5)}

This function allows redefinition of a window. The user will be asked for the window number to be redefined. Channels to be plotted and scaling on either axis may be changed. A window may be turned off by assigning channel -1 to the $X$-Axis. The information required is as described in Section 3.2.2 which describes the screen set up process. After entering the required data, the screen will be restored with the last one thousand data points replotted. If only one window is defined this function is immediate.

This function cannot change the number of windows allocated screen space. For example, if DATAVG was configured to use four windows, screen space is allocated in quarters. If only three windows are in use, this function can turn on the fourth window and it can turn off other windows but it cannot change the size of these windows. If DATAVG is configured to use three windows, the screen is divided into quarters as above. However, this operation cannot turn on the fourth window.

\subsubsection{Clear a Window (F6)}

This function clears all data from a specified window. A prompt will ask which window to clear. The valid range is displayed and inactive windows are rejected. This option is useful when data is of a cyclic nature and you need to see better detail of a given cycle. A 
window is redrawn but no data is plotted. If only one window is defined this function is imı nediate.

\subsubsection{Clear all Windows (F7)}

This function clears daia from all windows. All windows are redrawn but contain no data traces.

\subsubsection{Start a new Data File (F8)}

This option closes the current data file and starts a new ore with a new name. A prompt will ask for a file name and numiber of points as described above. No ".HDR" file is created. This is an extension of the test so the previous ".HDR" file is valid.

\subsubsection{End the Test (Fy)}

This function ends the test after confirmation from the user. The user must enter the leiter "Y" to end the test. All other responses will cause the test to resume. Data recording is stopped while waiting for the user confirmation.

No shifted or otherwise modified function keys are defined and any unused function keys are ignored.

\subsection{Recall Setup Data}

This operation asks for the name of a file containing setup data. A valid file name is required. If the name cannot be remembered, press the ESC key and DATAVG will return to the main menu. Exit from DATAVG and find the required name. After opening the file, setup information is read into internal data structures. DATAVG will tolerate setup files which are either too long or too short. A long file will be read until the maximum number of channels have been processed. A short file will leave channels undefined but available for use. The main menu is displayed on completion of this operation.

\subsection{Exit to DOS}

This operation returns to DOS after files are closed and the screen is cleared.

\section{TEMPERATURE CHANNELS}

Temperature channels are implemented as computed channels on a pseudo board named TEMP. This board requires the presence of an AIM7 interface board. Types $\mathrm{J}$ and $\mathrm{K}$ thermocouples are supported over a temperature range of -100 to +500 degrees Celsius using the tables supplied. The tables may be extended as needed by using the procedure in Section 5.4. The conversion from voltage to temperature is done by a cubic spline interpolation. Interpolation tables are loaded from a disk file (THERMO.CFG) when the first channel of this board is configured. If this file is not present in the current directory DATAVG will issue a message and temperature readings will not be allowed. 


\section{DISK FILES}

\subsection{Setup File}

Setup files contain all data needed to configure DATAVG fn: a particular job. Each field in the file will be described in this section. See Figure $i$ for an example setup file.

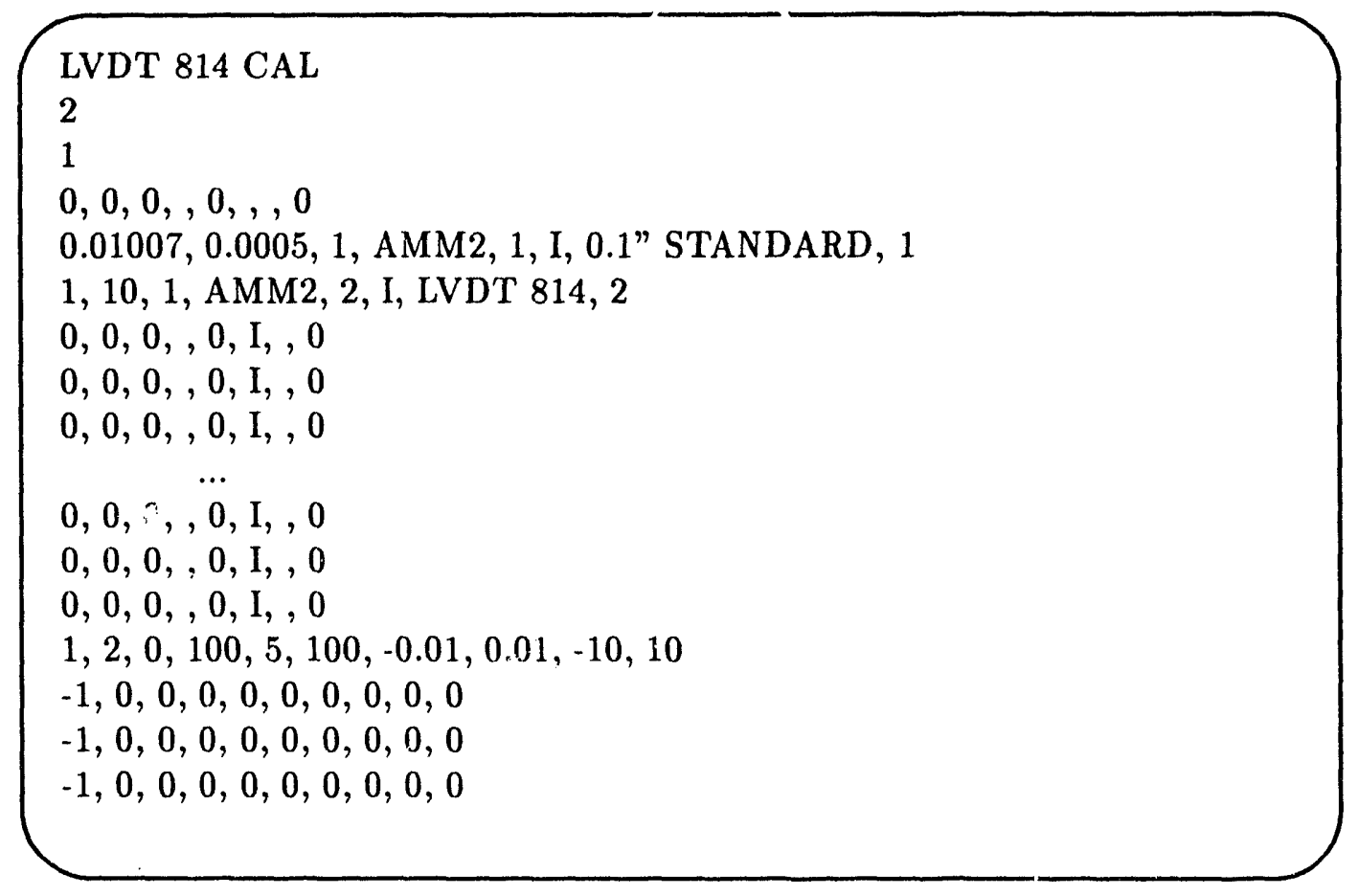

Figure 1: Setup File

The first line lists the test name. The second line contains the highest numbered active channel plus one and the third line contains the number of active windows.

Subsequent lines contain data for each channel. All avajlable channels are represented whether they are used or not.

Data are as follows with the fields separated by commas and spaces.

Column one, Scale factor in units per volt. Column two, Trigger threshold in scaled units. Column three, Flag indicating whether the first reading is to be the zero offset. Non zero values indicate a zero offset will be applied. Column four, The interface board name. Column five, The hardware channel number on this board. Coinmn six, A channel type character (I (in), O (out) or $\mathrm{C}$ (computed)). Column seven, The channel name. Column eight, The column number these data will occupy in the output file ( 0 if not in the file).

The last four lines contain data about the windows. This data is the screen positions and user unit ranges for both axes. The data is as follows with the fields separated by commas and spaces.

Column one, $\mathrm{X}$ axis channel. Column two, $\mathrm{Y}$ axis channel. Column three, Screen $\mathrm{X}$ axis minimum. Column four, Screen $\mathrm{X}$ axis maximum. Column five, Screen $\mathrm{Y}$ axis minimum. Column six, Screen $\mathrm{Y}$ axis maximum. Column seven, User unit $\mathrm{X}$ axis minimum. Column 
eight, User unit $\mathrm{X}$ axis maximum. Column nine, User unit $\mathrm{Y}$ axis minimum. Column ten, User unit $\mathrm{Y}$ axis maximum.

\subsection{Header File}

The header file (filename.HDR, Figure 2) starts with the version of DATAVG being used. The version includes the compilation date. The version information occupies the first two lines. Next comes a copy of the setup data as described in Section 5.1. There is a time stamp (including the date and time) followed by the column headings. Column headings are the names of each active channel. The offset data are stored in the last line of the header file. This offset information may be used to reconstruct actual readings if needed.

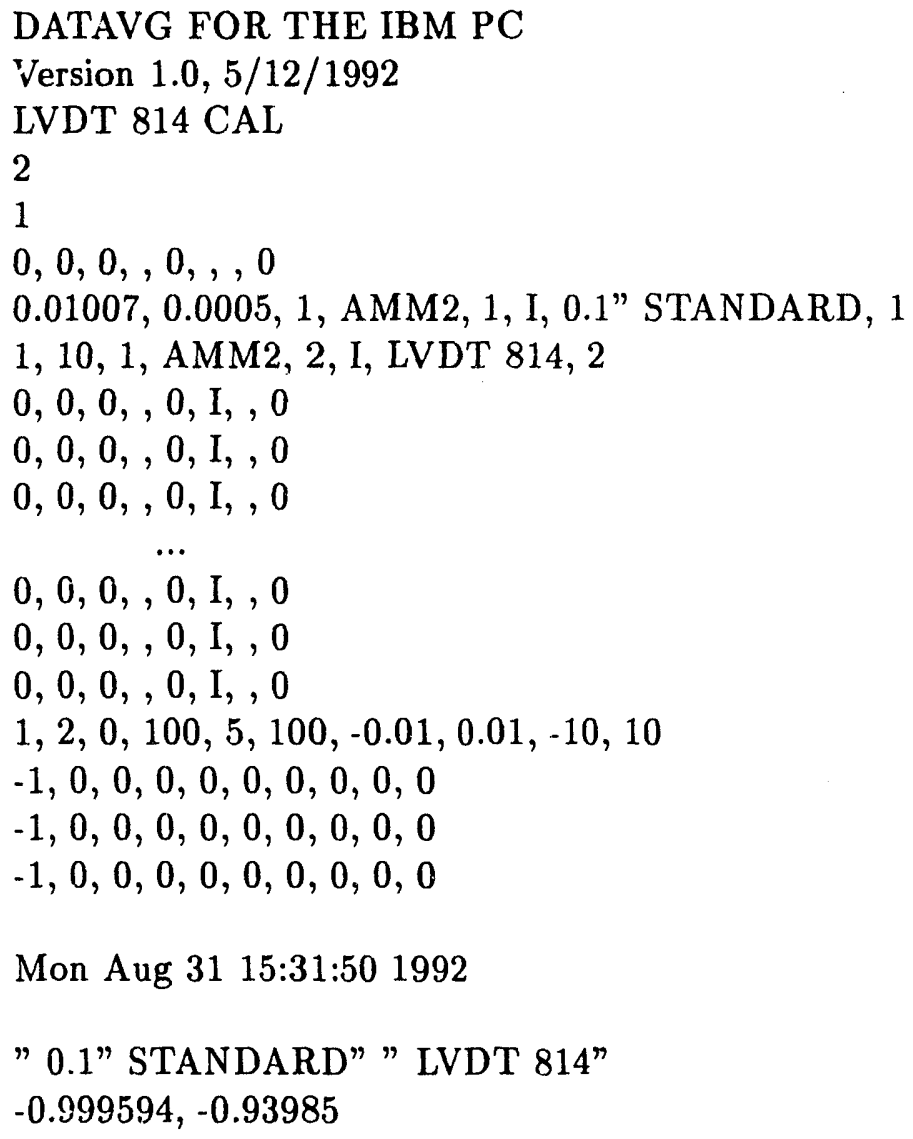

Figure 2: Header File

If a filename is re-used, the new header information will be appended to the existing file. It is easy to find the new header information by searching for the DATAVG program name in the file. 


\subsection{Data File}

Data storage starts in the second file (filename.DAT, Figure 3). Data are stored in ASCII real numbers with commas and spaces between columns. The data format may be floating point or exponential, whichever is shorter. Time data is expressed in elapsed seconds, since the start of test, in floating point format. All data from an event are stored in a single line. Lines are terminated by CR LF pairs as is customary in DOS systems.

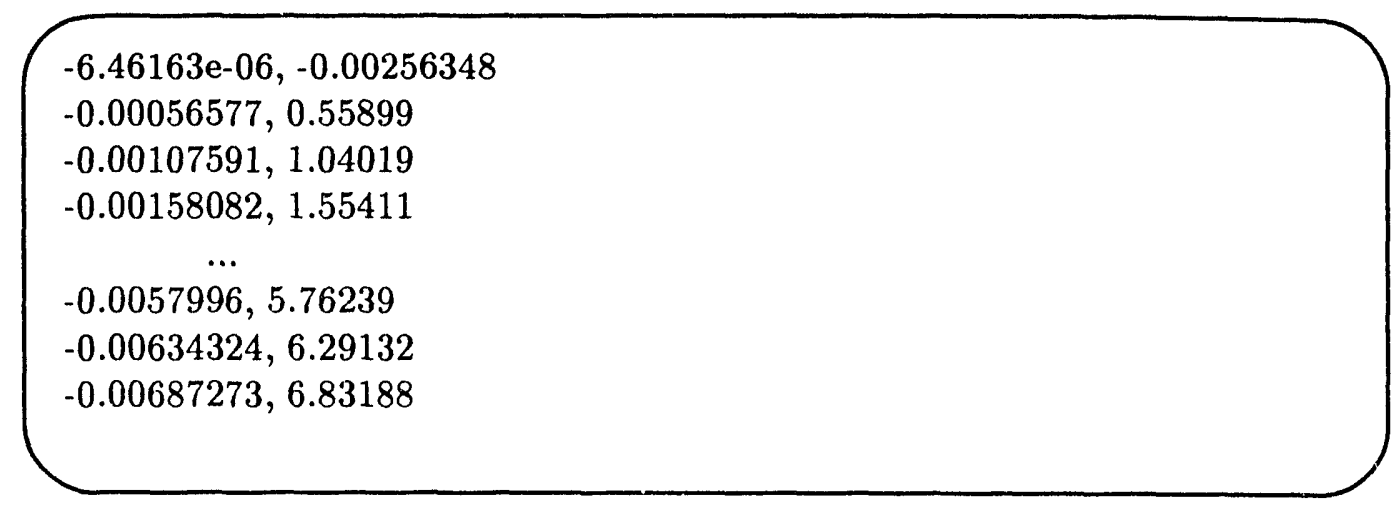

Figure 3: Data File

Data files (filename.DAT) contain data only. If a filename is re-used, data will be appended to the existing file. There is no blank line or other marker in the file to indicate where re-use started. It is possible to find the point where data was appended by looking for a jump, usually negative, in the time channel.

\subsection{Thermocouple Data Tables}

The tables in THERMO.CFG are created by a supporting program (BUILDTC, see Appendix G for source code), and are based on tables in the OMEGA temperature measurement data book Vol. 28. Tables may be created or extended by creating a file named TEMP.D containing temperature, millivolt pairs in ascending order of temperature.

In THERMO.CFG, the first line of a table must contain the thermocouple type letter, a comma and the number of temperature points in the list. Subsequent lines contain the AIM7 board output in volts referenced to zero degrees $\mathrm{C}$, a comma then the temperature in degrees Celsius, a comma and the cubic spline coefficient data. One data point is allowed on a line. Multiple thermocouple types may be contained in a file by concatenating them. While BUILDTC does not care how many types are contained in a file, only the first ten will be read by DATAVG. To conserve memory, the number of types should be kept to a minimum and only the types in use should be in the configuration file THERMO.CFG.

\section{INSTALLATION NOTES}

When installing DATAVG, a file named K500.CFG, Figure 4, must be created. It must contain the address of the interface board and the names, etc., of the boards in the Keithley box. This file is described in detail in Section 6.1. 
The first line contains the segment address of the 500-IBIN-A interface board. This board is memory mapped and must not conflict with other boards or DOS. If DOS 5.X or Windows is in use, it is possible to load system programs in high memory areas which may conflict with the factory default address of CFF8 HEX. Run the DOS command

MEM /D > PRN:

to print the memory map. If any programs start at addresses near CFF80, check for conflicts. Any ronflicts will require changing the board address, as described in the Keithley manual, to remove the conflict. In a GATEWAY $2000486 \mathrm{DX} 2 / 50$, setting the board to DFF8 works. DATAVG has not been tested with EMM386.

\subsection{Keithley Box Configuration I'ile}

The file K500.CFG, Figure 4, must be in the \DATAVG directory when DATAVG is started. This file contains the configuration of the Keithley 500 mainframe. K500.CFG must be maintained current for the specific computer. This is the only part of DATAVG which is specific to a particular computer.

The first line contains the segment address of the interface board expressed in HEX notation. When DATAVG starts, it reads K500.CFG and uses this address to initialize the interface board. Subsequent lines contain a list of the boards present.

Board list lines contain four fields separated by commas. The first field is the board name. Following the name is the slot number, channel count and board type. The type is Input or Output.

While newer Keithley 500 mainframes can identify the boards present, DATAVG does not currently make use of this feature. Instead, DATAVG uses the board list in K500.CFG.

\subsection{Temperature Data Files}

The temperature measurement system in DATAVG uses pre-computed data tables which are read from a file named THERMO.CFG. 'THERMO.CFG must be in the \DATAVG directory when temperature channels are configured. The file structure is discussed in Section 5.4.

D0F8

AMM2,1,16,I

AIM3,3,32,I

AOM $1,5,2,0$

DIO1,8,16,I

DIM1,9,16,I

PIM2,10,4,I

Figure 4: Keithley Configuration File, K500.CFG 


\section{LIMITATIONS AND REQUIREMENTS}

DATAVG uses 500LIB to interface with its hardware. The library is written with the assumption that a Keithley 500-IBIN-A interface board is present. This board contains the counters and clock used to time events. The older 500-IBIN board will not work since it does not include a crystal oscillator. The analog input routines assume a sixteen bit $A / D$ converter on a Keithley 500-AMM2 board. If different hardware is needed, 500LIB must be modified and recompiled.

\subsection{File Manageinent}

Data are recorded whenever the defined threshold is crossed on any channel. Data are writtun to disk when they are plotted on screen. A power failure during a test could cause the loss of any data written to the file. Closing the file assures that the disk directory will be updated in a timely manner which reduces chances of data loss. Therefore, for data security, the number of data points recorded in a file before closure is limited. When this limit is reached the file is closed and reopened. Subsequent data are appended to the file, and this process is repeated until the end of test. There is a limit of 65536 data points before closing the data file.

\subsection{Screen Management}

All plots are updated to reflect the latest data written to disk. This update does not wait for the file closure discussed in Section 7.1.

\subsection{Time Interval Triggering Limits}

The time data used for triggering is stored in a float. This supplies sufficient precision to allow triggering at one-second intervals for about ninety days. If a test runs longer than this, trigger at longer intervals. Actually, one-second intervals would be rather short for a test of this length so the loss of precision should not be a problem.

\section{COMPUTED CHANNEL DESIGN}

The remainder of this document is primarily of interest to programmers. The user probably will not need to read further. This section is included to document the program structure.

\subsection{COMPUTED CHANNEL INTERFACE}

Computed channels are implemented using object-oriented programming techniques. They are treated by DATAVG as if they resided on boards. Pseudo boards may be defined which have needed properties. These boards may support multiple channels like a real board. Each channel of a pseudo board must return a computed value based on real readings and/or other computed channels.

The user may write computed channel drivers and link them into the program by making proper references in the MAKEFILE. This requires adherence to the function prototype to 
avoid compiler complaints. User-written drivers must take care of any required initialization. User-written drivers must \#include "DATAVG.H". If using Borland MAKE, create a file named DATAVG.LNK containing the linker instructions.

Computed channel drivers must keep static variables or arrays containing information used for their calculations. They must, also, handle four functions signified by the card.slot variable. Card_siot values of $-1,-2$ and -3 specify special functions as described below.

Another rer uirement is to place the function prototype near the beginning of DATAVG.H so the compiler can reference it. Include the board name, slot number, channel count, type and driver pointer in the box[] array found in DATAVG.H. When installing the pseudo board, choose a slot number greater than ten and within the limits of the box[] array, (currently 15 as defined by MAX_SLOT). Board names should be seven or less characters.

\subsubsection{Computed Channel Driver Function Prototype}

The function prototype for computed channel drivers is: float name(int channel, int card_slot, int num_to_ave).

\subsubsection{Initialize Computed Channel}

A card_slot value of -1 signifies a need to initialize this channel. This function may request any information required to perform the required operation such as scale factors and input sources.

In the setup_channels function (Section 3.1), the user is asked for data about each channel. Part of this data is the board name and board channel to be used for this program channel. If the board is a pseudo board, the board driver will be called to do any required initialization. The initialization request will be signified by a card_slot number of -1 . When making the initialization call, the driver will be given the pseudo board channel to be configured. The num_to_average variable will contain the program channel number being processed. The program channel being processed is useful in detecting references to other channels which will contain obsolete data at the time this channel is evaluated. Only channel numbers less than this one can provide currently valid data from the $\mathrm{D}[\mathrm{l}$ array.

Computed channel drivers may use previous channel data, or they may make independent readings from the interfaces. During set up, it is possible to test references to other channels to see if they have been defined. This may be done by testing their scale variable (channels[n].scale). Channels which are in use have non-zero scale factors. References to undefined channels should be treated as warnings, since they may be defined later. At run time, references to undefined channels should be flagged as errors and the run stopped.

During configuration of a computed channel, a physical channel may be selected by a call to get_valid_board(). This function requires a pointer to the prompt text. It returns an index into the box[] array where information about the board is located. get_valid_board() also returns the selected channel on the board. The driver element of the box[] array entry may be called to make a real reading. Several real readings may be taken as needed by calling the appropriate drivers. This data may be combined with data from previously read channels as needed. Previously read data is in the D[] array and may be indexed by the channel number. Obtaining data from the $D[]$ array is much quicker than making new reauings. It is possible to write drivers which use new readings only when needed. 


\subsubsection{Save Computed Channel Set Up Data}

The savessetup() function (Section 3.5) in DATAVG calls computed channel drivers during its operation. save setup() calls the driver with the pseudo board channel number and a slot number of -2 . The driver must write any required data to the setup file. This operation is needed because DATA.VG has no way to know what this driver does or how it is done. Drivers normally maintain private data which needs to be restored before operation can resume. The file pointer to be used is data_file and the information should be saved in ASCII with a newline at the end.

\subsubsection{Restore Computed Channel Configuration}

The restoresetup() function in DATAVG (Section 3.8) calls computed channel drivers during its operation. restore_setup() calls the driver with the pseudo board channel number and a slot number of -3 . The driver must read saved set up data from the setup file, using the file pointer data_file, and rebuild its internal tables. User prompts may be made for other data as required.

\subsubsection{Normal Computed Channel Operation}

During data collection, the drivel for a computed channel will receive a card slot number as shown in channels.card_slot, the channel number for this board and the number of readings to average. The number to average is a compile-time constant and is probably meaningless in a computed channel context but is included for a consistent interface.

The driver must perform its function and return a float result. If data from lower numbered channels is desired, it may be obtained from the $D[]$ array. The channel number is the index into this array.

\subsubsection{Support Functions}

There are several support functions available to writers of computed channel drivers. These include functions to prompt for and validate numerical data and board identification. Use of these functions can make your code smaller and easier to write.

8.1.6.1 Get an Integer with prompt and default. get_int() returns an integer. It requires a prompt string and default value. Input is validated to insure the correct type. and the default is returned if the user presses ENTER alone. See the prototype in DATAVG.H for details of this function.

8.1.6.2 Get a Float with prompt and default. getfloat() returns a float. It requires a prompt string and default value. Input is validated to insure the correct type, and the default is returned if the user presses ENTER alone. See the prototype in DATAVG.H for details of this function. 
8.1.6.3 Get a Board and Channel. get_valid_board() requires a pointer to a prompt string. It asks for and obtains a board and channel identification. This function returns an index into the box[] array. By using this index, a description of the selected board may be located. This function also returns a channel number on the board. See the prototype in DATAVG. $H$ for details of this function.

8.1.6.4 Validate a File Name. validate_file_name() requires a pointer to a string containing the file name to be validated. All characters DOS does not like are removed and the remainder are concatenated in the original string. The name and type fields are truncated so they do not exceed their maximum lengths. This function removes control and illegal characters but does not case shift the string.

\section{PROGRAM STRUCTURE}

DATAVG, written in Turbo $C$ for the IBM PC family, is derived from a program of the same name which ran on a Digital PDP-11. The original, written in MTS BASIC, was severely limited by the hardware environment. Using the PC allows greater flexibility in interfacing and display hardware and larger memory.

DATAVG currently operates with a Keithley 500 data acquisition system. A Keithley 500-IBIN-A interface board is REQUIRED since it contains the clock circuits used for timing events. A Keithley 500-AMM2 board is assumed, but a 500-AMM1 could be used with minor program changes, as could the Keithley 570 or other hardware.

DATAVG supports computed channels which are implemented using object-oriented programing techniques. Computed channels may perform nearly any function required including experiment control. In this implementation of DATAVG, there is a temperature board which is implemented using computed channels. See Section 8 for instructions on writing your own drivers.

Display hardware which may be used includes IBM CGA, EGA, VGA and IBM8514 color plus Hercules monochrome. EGA, VGA and 8514 are definitely preferred. When using a color interface board, a color monitor MUST be used. There is no support for gray scale on a monochrome VGA system.

DATAVG source code is contained in the file DATAVG.C (Appendix A for DATAVG.H, and Appendix B for DATAVG.C). This module requests inclusion of DATAVG.H which includes any required standard header files and a few locally-created header files. Locallycreated headers are 500LIB.H and SRBGRAPH.H. DATAVG.H contains definitions and data declarations for the program. 500LIB.H and SRBGRAPH.H provide function prototypes for their respective modules.

DATAVG uses 500LIB to interface with the hardware. This is a driver package written in Turbo $\mathrm{C}$ which makes some assumptions about the available equipment. This library was not intended to be as comprehensive as the Keithley-supplied driver package. It is assumed that there will be a 500-IBIN-A interface board or equivalent which provides the timing equipment. Further, it is assumed there will be a 500-AMM2 in slot one of a Keithley 500 mainframe. By changing some definitions and recompiling the library, it is possible to support a 500-AMM1. Source code for 500LIB.H is in Appendix C and 500LIB.C is in Appendix D . 
In writing DATAVG, efforts were made to keep the code modular and to eliminate special cases. Channel zero (TIME) requires some special treatment since no scaling is done. Also, computed channels are "Black Boxes" which may have private data. This requires that computed channel drivers be able to do their own setup. They must, also, be able to save and restore their own configuration.

Functions were written so they could be used in several places throughout the code. Some functions are used to set up the parameters for a call to another, reusable, function. In a few cases, the use of flag variables allowed changing the behavior of functions for initialization vs. normal use.

In user-interface functions where speed is not needed, functions were used to make the code more readable. This was done where the same type of information was requested in several places and verification was needed.

In the user-interactive routines, extensive range checking has been implemented. When input is requested, the data is checked to prevent use of invalid information. For instance, if a number is needed the program will not allow alphabetic entries. Most prompts include acceptable ranges, and these are tested before DATAVG will continue.

Routines which read files created by this program do not contain extensive range testing. It was assumed that data in files created by DATAVG, including setup files, had already been range checked and was correct. While it is possible to modify or create setup files with a text editor, there is virtually no error checking while reading them. Be careful when editing setup files.

DATAVG uses SRBGRAPH to do plotting. This is a graph package written by S. R. Brown and modified by R. D. Hardy (both Sandia National Laboratories). SRBGRAPH is a very modular library of object modules which may be linked into any program which requires X-Y graphing capabilities. See Appendix E for the header SRBGRAPH.H and Appendix $F$ for composite source code.

\section{VARIABLES}

This section discusses the global variables used by DATAVG.

\subsection{Global General Data}

char BUFFER [64];

This is a character array used for data entry from the keyboard. In the functions which interact with the user, most data are read in string form and later parsed into the required form.

char Test_ID [64];

This string holds the test identification which is placed in the data file header.

char Data_file_name[64];

This string holds the complete data file path. Space is allowed for the longest path DOS can handle. 
int num_chan;

This is the highest program channel number in use. It is not the number of channels. This variable appears in line two of the setup file.

int Points_this_file;

The number of data points recorded since opening the data file. This variable is used to decide when we need to close and reopen the data file.

int Poin_us_per_file;

The number of points to store before closing and reopening the data file.

long Total_points;

Total number of data readings recorded in this test.

int plot_data_count;

This variable is not used as its name implies. It is an index into the channel data storage buffer. This variable is used by the plot function and to compare current data against previously stored data.

int plot_windows; file.

The highest numbered window in use. This variable appears in line three of the setup unsigned int SEQUENCE;

A flag used to show whether we are storing data or the data header.

\section{float D [MAXCHAN+1];}

This global array contains the current data readings. During data scanning, this array contains data for all channels which have been read. Higher numbered channel data is not valid. Computed channels may use data from this array as needed.

FILE *data_file, *printer;

File pointers. 


\subsection{Time Data}

struct ktime Ktime;

A structure defined in 500LIB.H which is used to return time codes from the Keithley interface board.

double base_time;

This variable holds the time when data recording started. This value is subtracted from all times to get relative time from the start of recording.

double rel_time;

This variable holds the time from the Keithley board relative to program start. The Keithley board timer is started during program initialization and can contain a significant value before recording starts.

struct timeb start_time;

A structure defined in /TC/INCLUDE/SYS/TIMEB.H. This structure is used to return DOS system time which is used to time stamp the data file header.

\subsection{Plot Data}

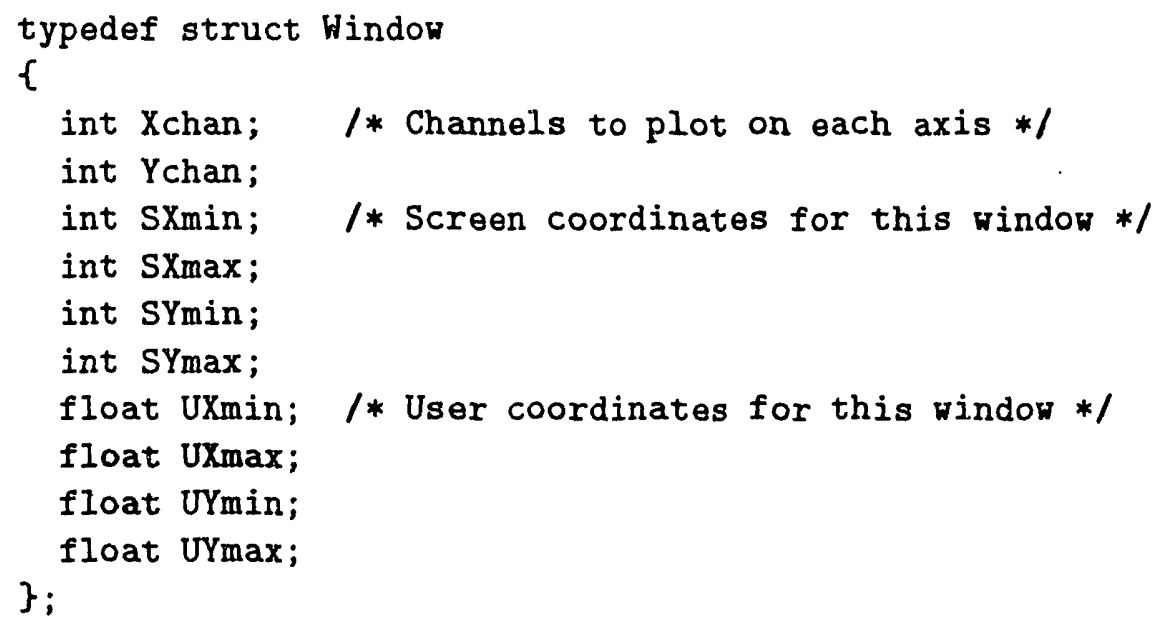

This structure defined in DATAVG.H contains all required data about a given window.

struct Window win_data[MAXWINDOWS];

An array of window data structures with one entry for each window. A maximum of four windows is allowed in the current version, but this could be changed if required. Four windows are a good trade between visibility and the amount of data we could display. 


\subsection{Program Channel Data}

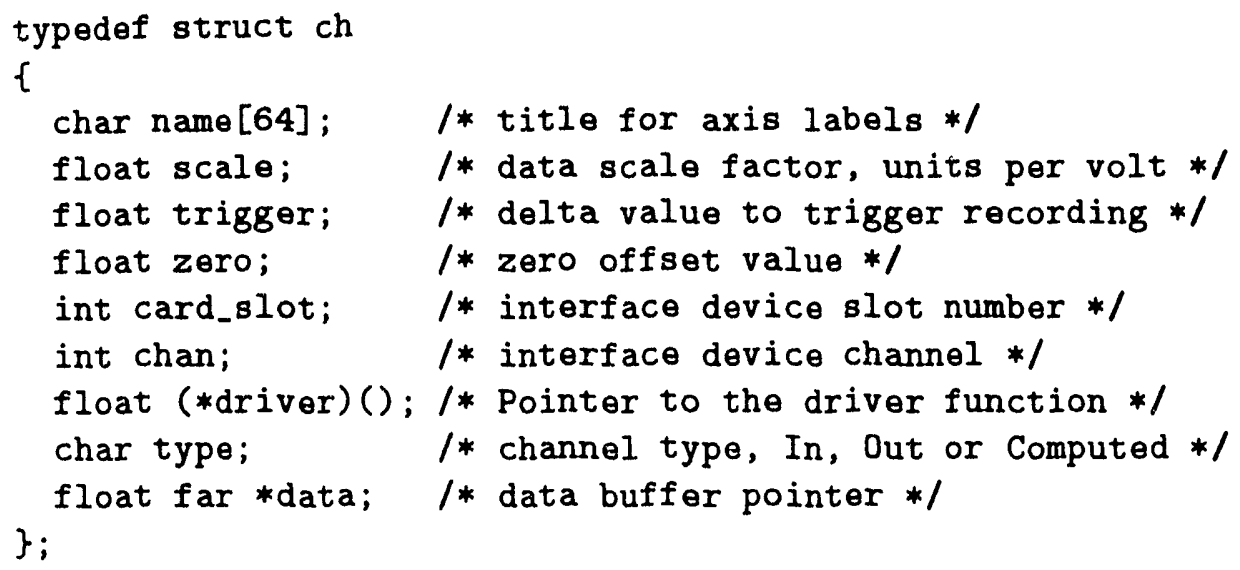

This structure defined in DATAVG.H contains the data required for each program channel. It can be used for either real or computed data channels.

struct ch channels [MAXCHAN+1];

An array of channel data structures. There is one entry for each prugram channel allowed. Currently there are thirty channels but this could be increased if needed.

\subsection{Hardware Information}

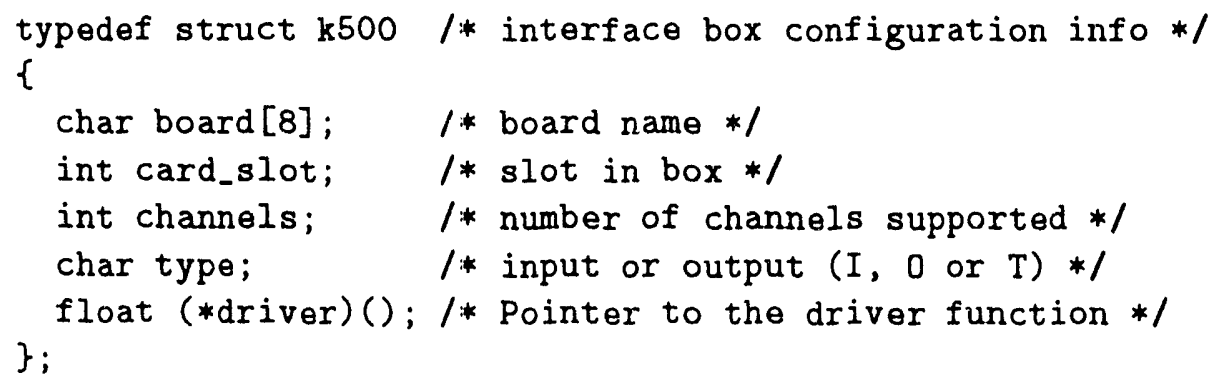

The box[] array of structures defined in DATAVG.H contains data for each Keithley 500 board present. This structure is also used to hold information about pseudo boards supporting computed channels starting at card slot 11. There is an entry for each real and pseudo board. Currently there are fifteen entries, but this could be changed.

struct $\mathrm{k} 500$ box [MAX_SLOT + 1];

The boards data structure defined in DATAVG.H contains a master list of all Keithley boards and their characteristics. It is used during auto-identify and to hold driver pointers. Limited to 35 boards by hardware limitations. This array of structures is initialized at compile time. 


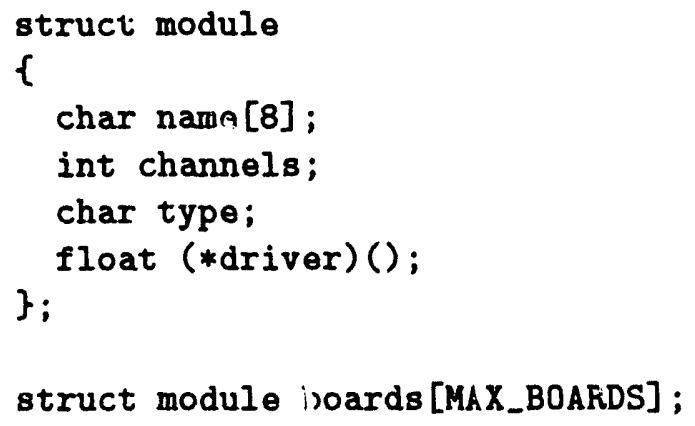

\subsection{Other Variables}

There are local variables used in various functions. The variables $i, j, k$ and $n$ are frequently used for loop counters and indexes. Other variables are named and commented so their function is obvious.

\section{END OF VARIABLES LIST}




\section{APPENDICES}

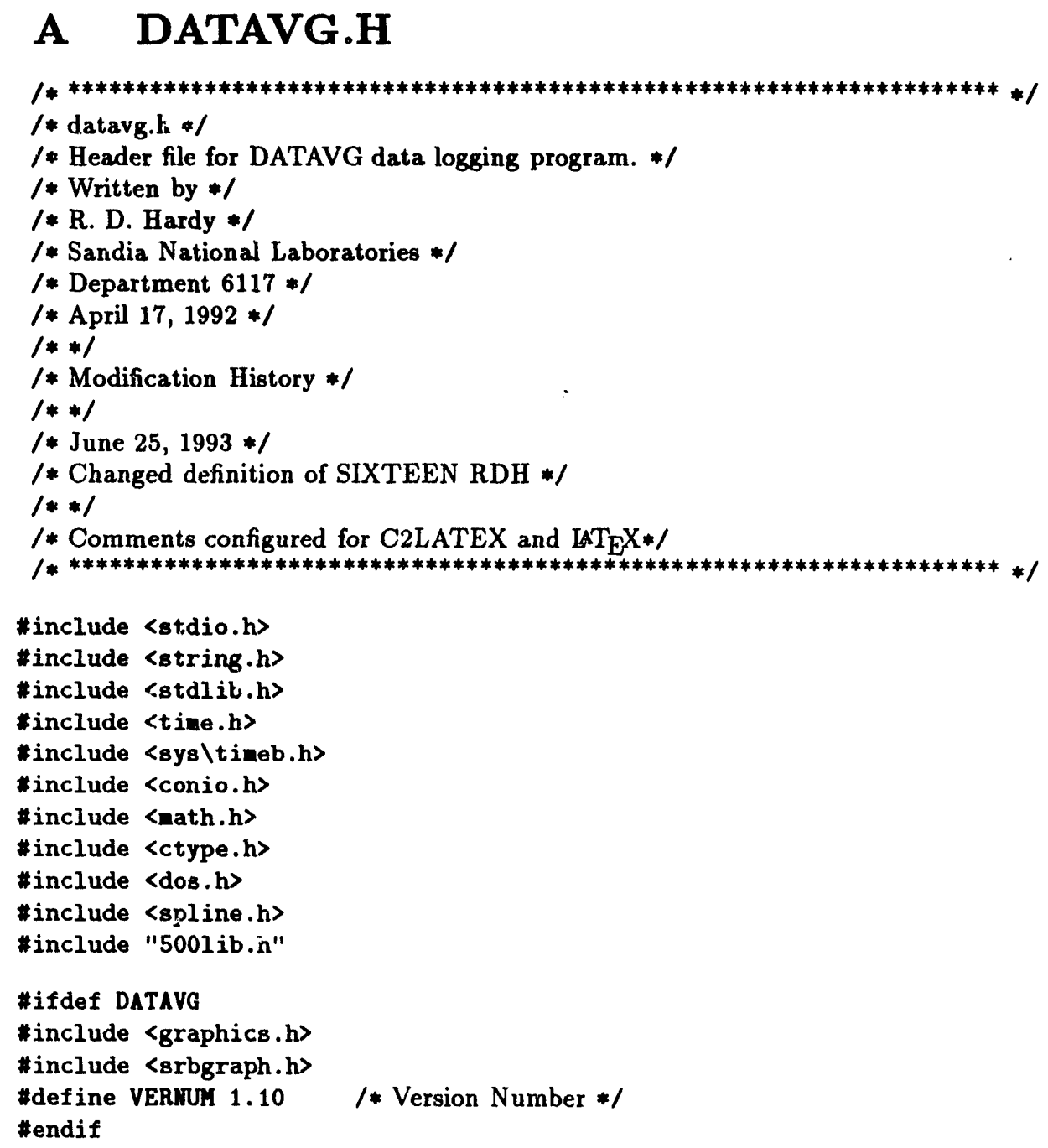

/* MAX_SLOT is the number of boards allowed including psudo-boards */ \#define MAX_SLOT 15

/*Other constants */

*define MaX_MODULES 35

*define MS_COUNT ((unsigned int) 1000)

*define SEC_COUNT ((unsigned int)1000)

/* number of entries in boards array */

*define Zoom_Scale 0.75

* counter 1 value, one second $* /$

/* counter 0 value, seconds counter */

/* Window zoom scale factor */

/* global function prototypes */

void validate_file_name(char *name);

void get_valid_board(char *text, int *box_index, int *chan);

float get_float (char *prompt,float def);

int get_int (char *prompt,int def);

float (*find_driver_pointer (char *name)) ();

char *get_board_nane (float (*driver) ()$)$; 
/* computed channel prototypes */

float axial_stress (int chan, int card_slot, int $n$ );

float thermocouple(int chan, int card_slot, int $n$ );

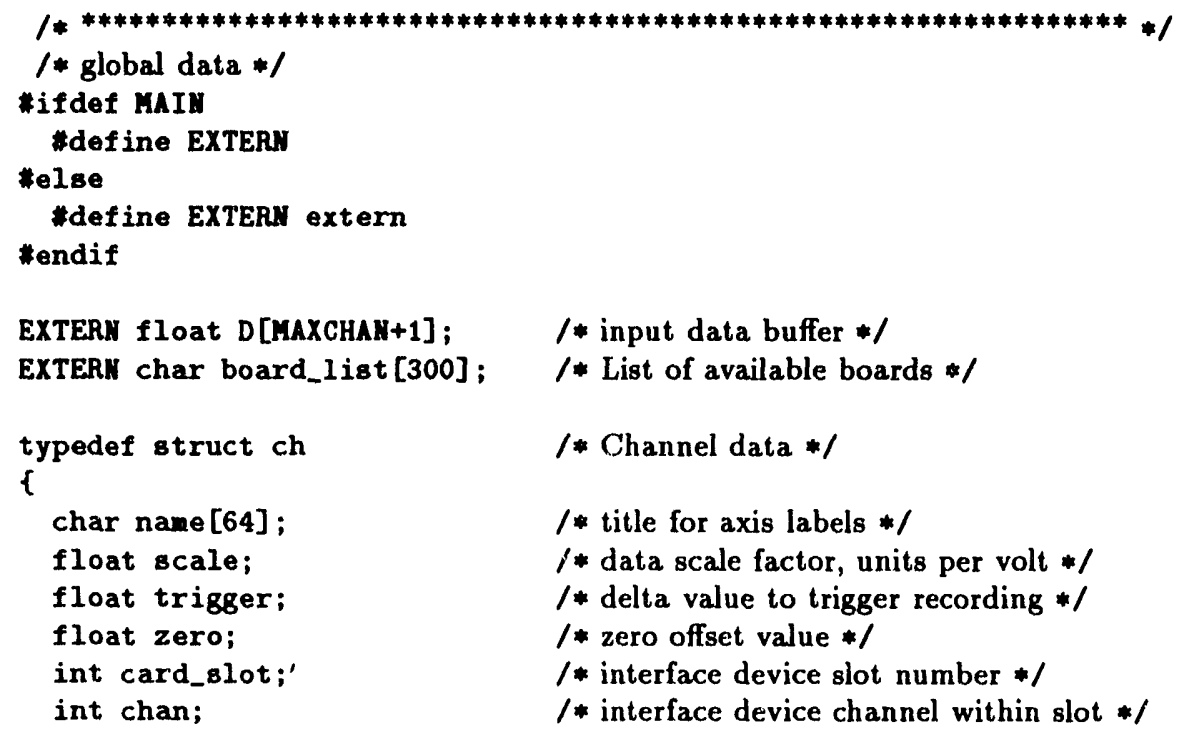

\section{*ifdef MaIy}

struct k500 box[MAX_SLOT + 1] =

\{

1* Card slot zero is not used, it is a dummy only */

* this space will be filled in during program startup */
$\{"$ "', $0,0,-1, '$, wULL $\}$,
$\{$ '"', $1,0,-1, '$ ', NULL $\}$,
$\{1+1,2,0,-1, '$, ,WULL $\}$,
$\{" \prime \prime, 3,0,-1, '$, , MULL $\}$,
$\{" \prime, 4,0,-1, '$, , NULL $\}$,
('i', $5,0,-1$, , ', NULL $\}$,
$\{" \prime \prime, 6,0,-1, '$, , NULL $\}$,
\{"'", $7,0,-1, '$, NULL $\}$,
$\{" 1 ", 8,0,-1$, , ', NULL $\}$,
$\{" \prime \prime, 8,0,-1, '$, ,NULL $\}$,
\{"', $10,0,-1$,' ', NULL $\}$,

/* dummies for real card slots */ 


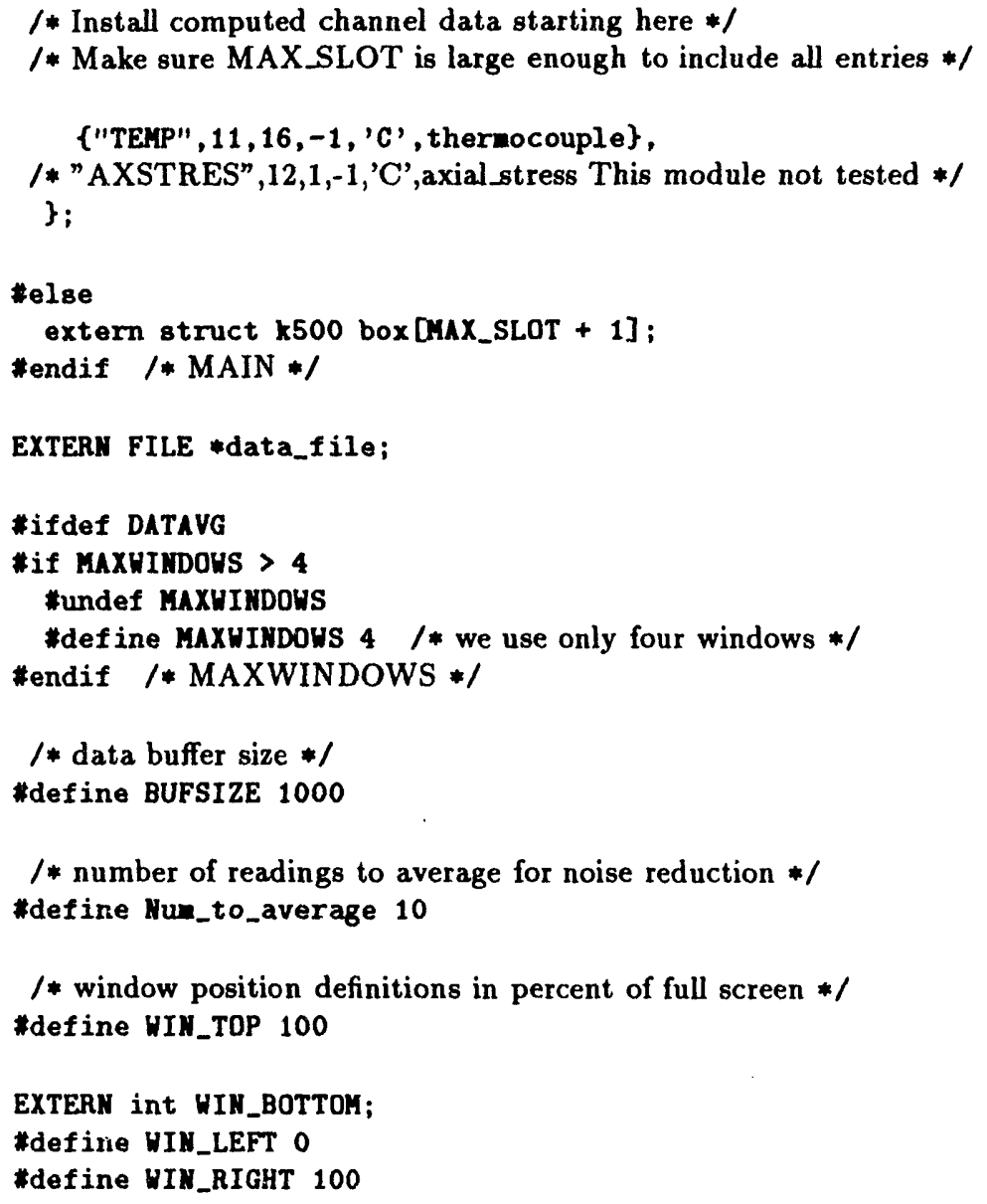




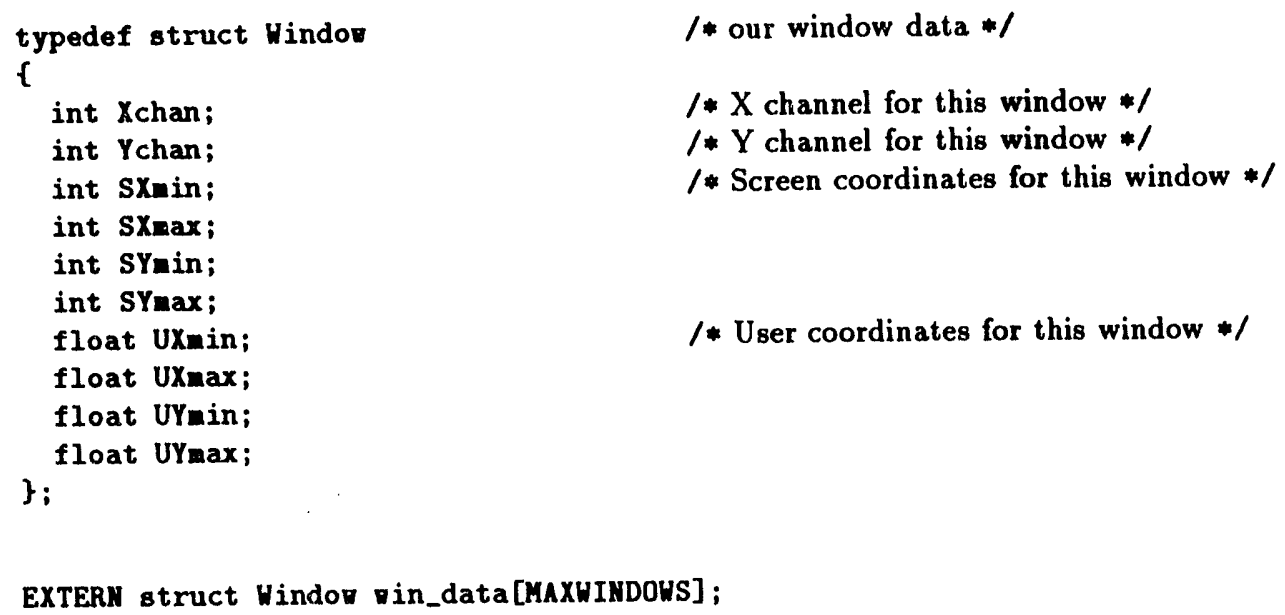

EXTERM struct Windor vin_data[MAXWINDOWS];

/* master data structure used during auto-identify and to hold */

/* driver pointers */

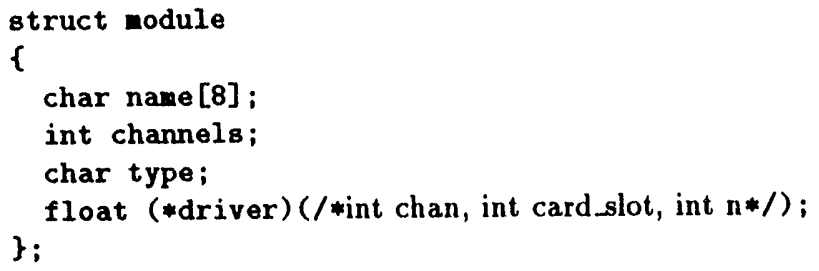

\section{*ifdef MAIr}

struct module boards[MAX_MODULES] =

\{

\{"ARAA1A", 1 , ' I', MULL \},

\{"AMI1", 1, 'I', MULL\},

\{"AMM2", 16, 'I', read_AMM2\},

\{"AIM2", 2, 'I', NULL\},

\{"AIM3", 32, 'I', read_AIM3\},

\{"AIM4", 1 , 'I', NULL\},

\{"AIM5", 1, 'I', NULL\},

\{"AIM6", 1, 'I', NULL\},

\{"AIM7", 16, 'T', read_AIM7\},

\{"AIM8", 1, 'I', NULL\},

\{"AIM9", 1, 'I', NULL\},

\{"PIM1", 2 , 'I', NULL\},

\{"PIM2", 4, 'I', read_PIM2\},

\{"STEP1", 1, 'O', NULL\},

\{"STEP2", 1, ' 0 ', MULL \},

\{"AOM1/2", 2, 'O', write_AOM1\},

\{"AOM1/5",5,'O', घrite_AOM1\},

\{"AIM2/1", 1, 'I', MULL\},

\{"AOM2/2", 2, 'O', NULL\},

\{"AOM3", 2, 'O', NULL\},

\{"AOM4", 2, 'O', NULL\},

\{"DIM1",16,'I', read_DIM1\},

\{"DOM1", 16, 'O', MULL\},

\{"DIO1",16, 'O', write_DIO1\}, 


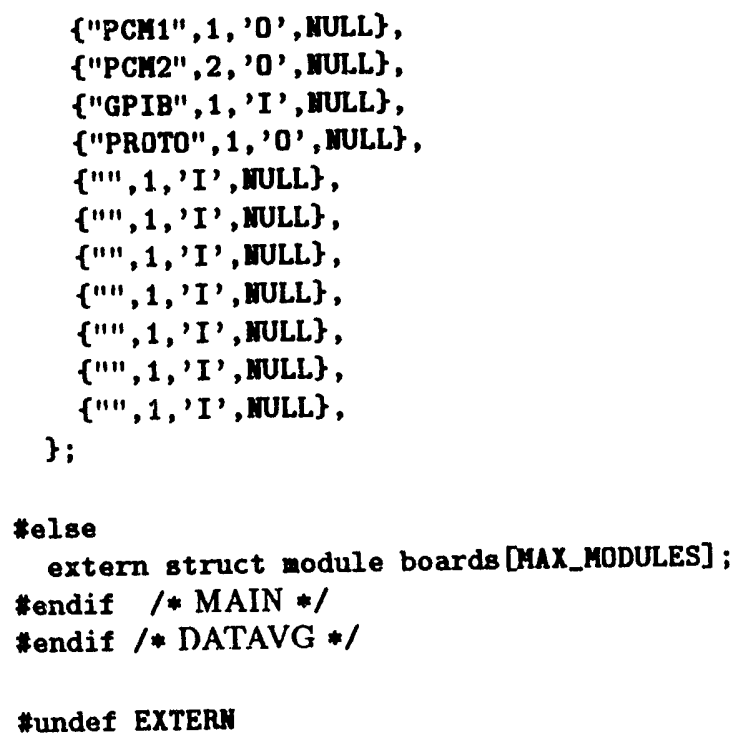




\section{B DATAVG.C}



\#define MaIN

\#define DATAVG

\#include "datavg.h"
/* MAIN includes the global data in this module */ /* Includes our definitions, other programs don't get them. */ /* define global data, DO NOT MOVE THIS LINE */

/* static function prototypes */

static int init_channels(void);

static void shor_setup(void);

static void gen_shor_setup(FILE *dev);

static void get_file_name(void);

static void check_channels (void);

static void get_data(void);

static int proc_function_key (int key); 
static int get_vindor_number(void);

static int save_setup(void);

static void orite_setup(void);

static void get_setup(void);

static void datavg_init (void);

static void get_box_configuration(void);

static void de_init (void);

static int test_kb(void);

static void ner_line(void);

static void read_data(int num_chan);

static void vrite_data(int flag);

static void redran_vindovs (int flag);

static void plot_data(int flag);

static void ny_init_graph(void);

static void setup_rindor(int vindor);

static void def_windor(int windor);

static void redo_vindor(int vindow);

static void zoom_in_vindor(int vindor);

static void zoom_out_rindor(int rindor);

static void zoom (int windor, float scale);

static void clr_vindov(int vindor);

static void set_driver_ptr(int slot);

static float timecode(void);

static int open_data_file(void);

static char Version [64];

/* Program version string */

static int MaxY;

/* screen height $* /$

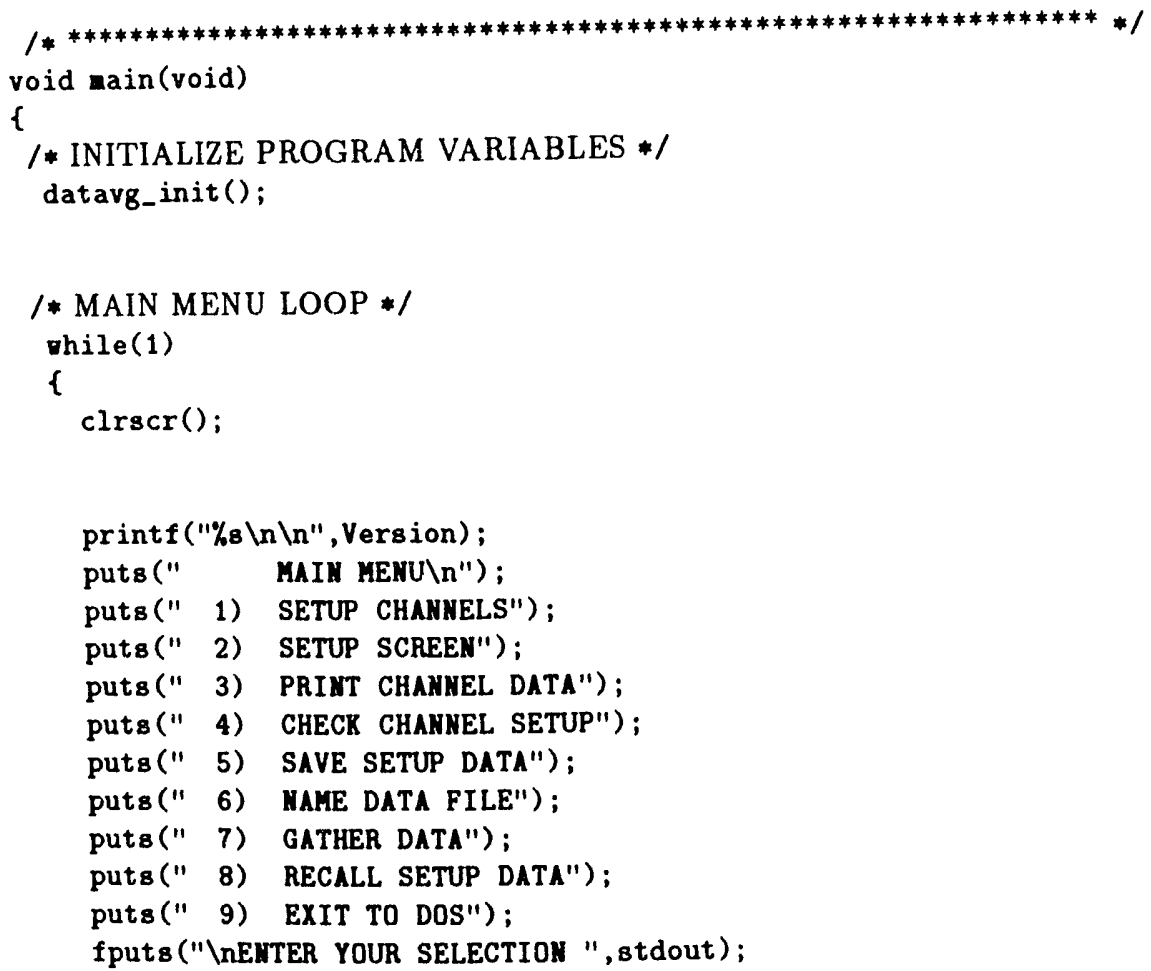




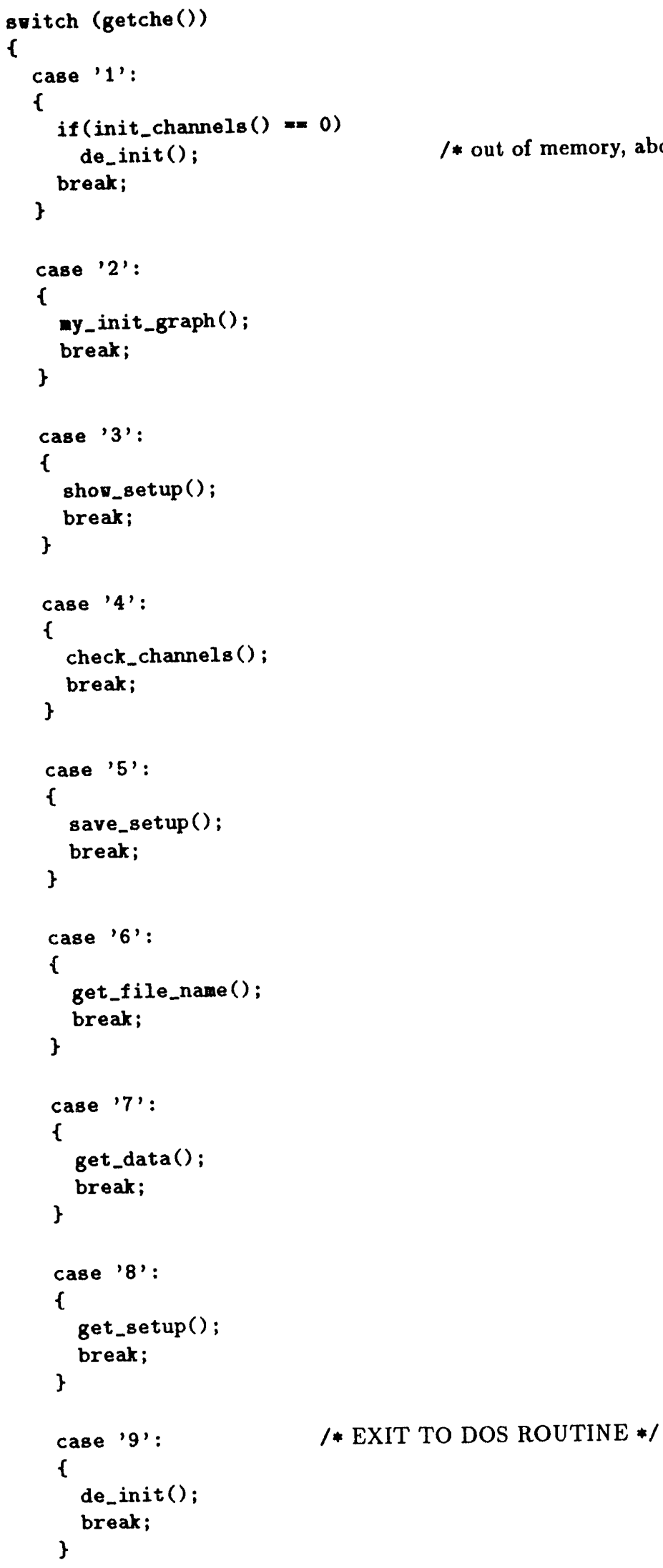




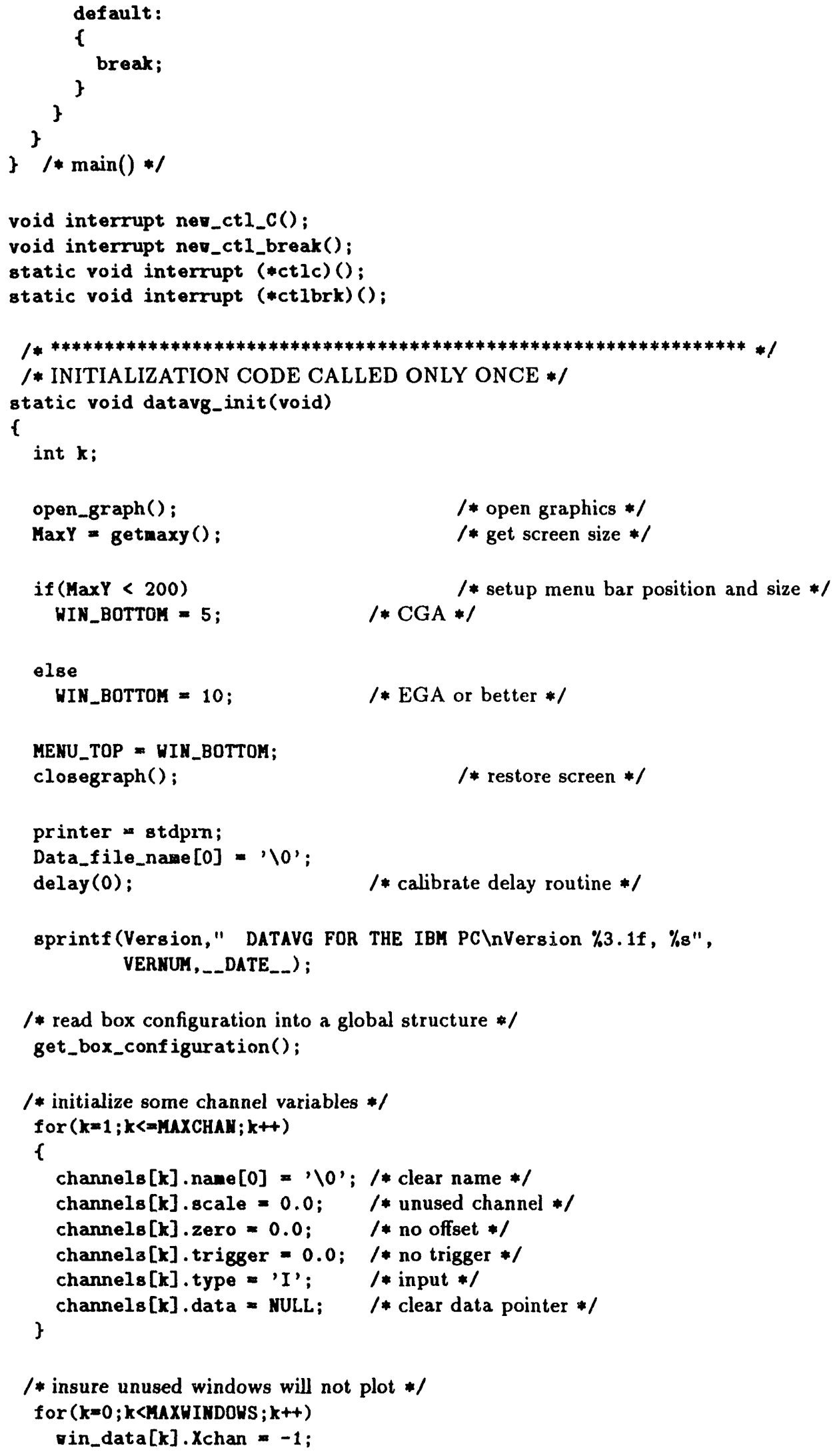




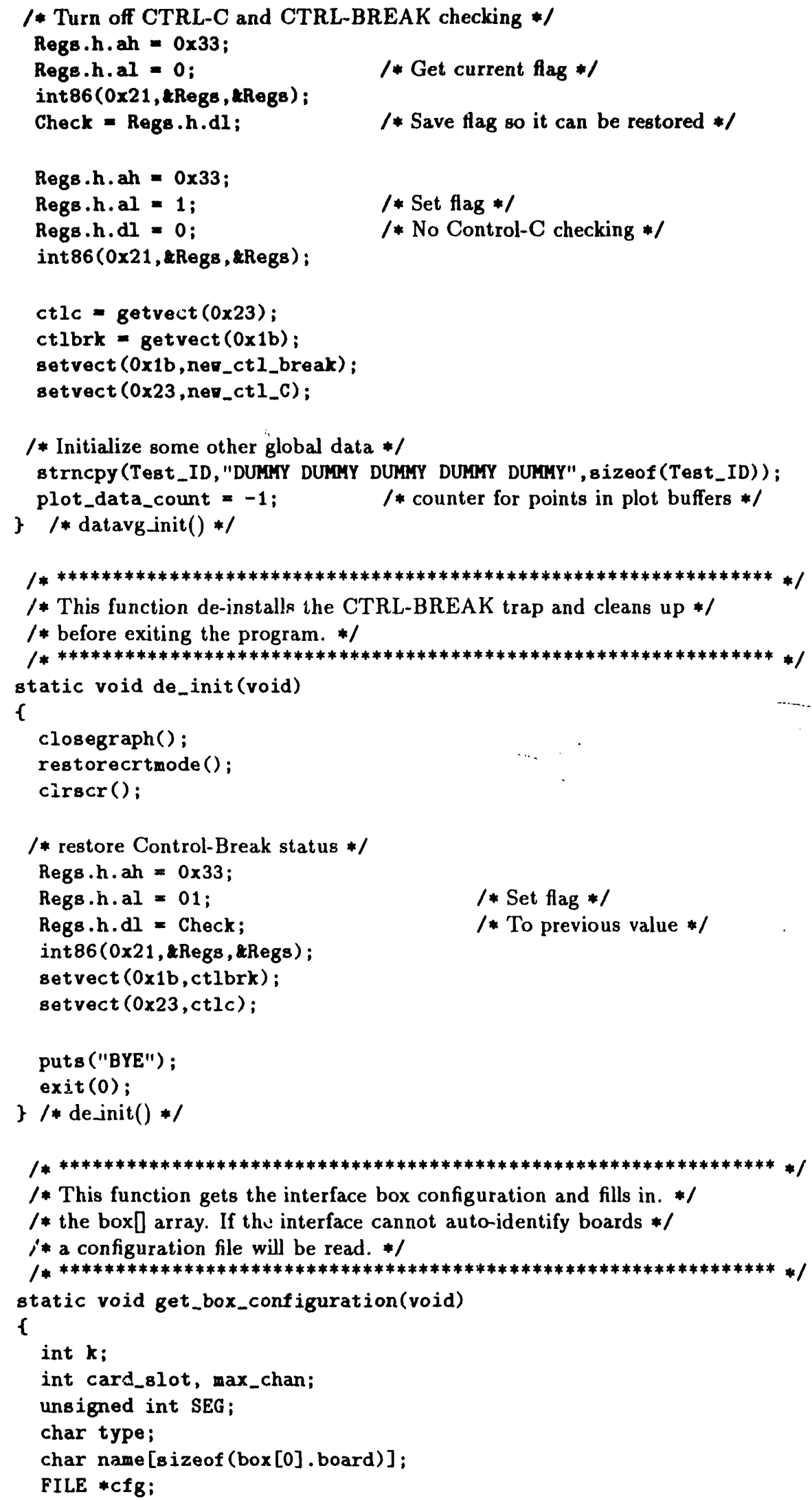




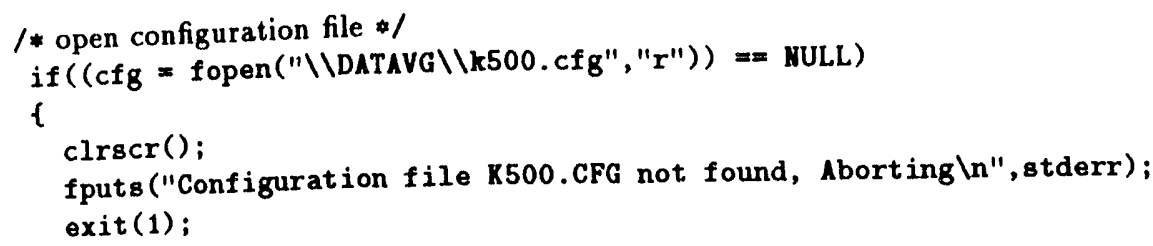

/* read segment address from file and initialize the interface $* /$ fgets (BUFFER, sizeof (BUFFER), cfg); sscanf (BUFFER, "\%x", $\angle S E G$ );

init_500(SEG); /* init hardware */

set_timer(MS_COUNT, SEC_COUNT);

/* set the counters */

/* read configuration from the file $* /$

for $(k=1 ; k<=10 ; k++)$

\{

if ( $f$ gets (BUFFER, sizeof (BUFFER), cfg) $==$ NULL)

break;

strtok (BUFFER,",");

strncpy (name, BUFFER, sizeof (box [0] . board));

sscanf (strtok (NULL, "\n"), "\%d, \%d,\%c", \&card_slot, kmax_chan, \&type);

strcpy (box [card_slot] . board, name);

box [card_slot], card_slot $=$ card_slot;

box[card_slot]. channels = max_chan;

box[card_slot].type = type;

box $[k]$. last_chan $=-1$;

set_driver_ptr(card_slot); /* put driver pointer in box $] * /$

fclose $(\mathrm{cfg})$;

\} /* get_box_configuration ()$* /$

$/ * * * * * * * * * * * * * * * * * * * * * * * * * * * * * * * * * * * * * * * * * * * * * * * * * * * * * * * * * * * * * * * * * * /$

/* load driver pointers into the box structure. */

static void set_driver_ptr(int slot)

f

int $i$;

/* look up board name in master list */

for $(i=0 ; i<$ MAX_HODULES; $i++)$

if $(\mathrm{strcmp}($ box $[\mathrm{slot}]$.board, boards $[i] \cdot \operatorname{name})=0$ ) break;

if ( $i=$ MAX_MODULES) $_{-}$

return; $\quad / *$ name not found */

/* get data about this board and place in box structure */

box[slot].driver = boards [i].driver;

\} / $*$ set_driver_ptr()*1 


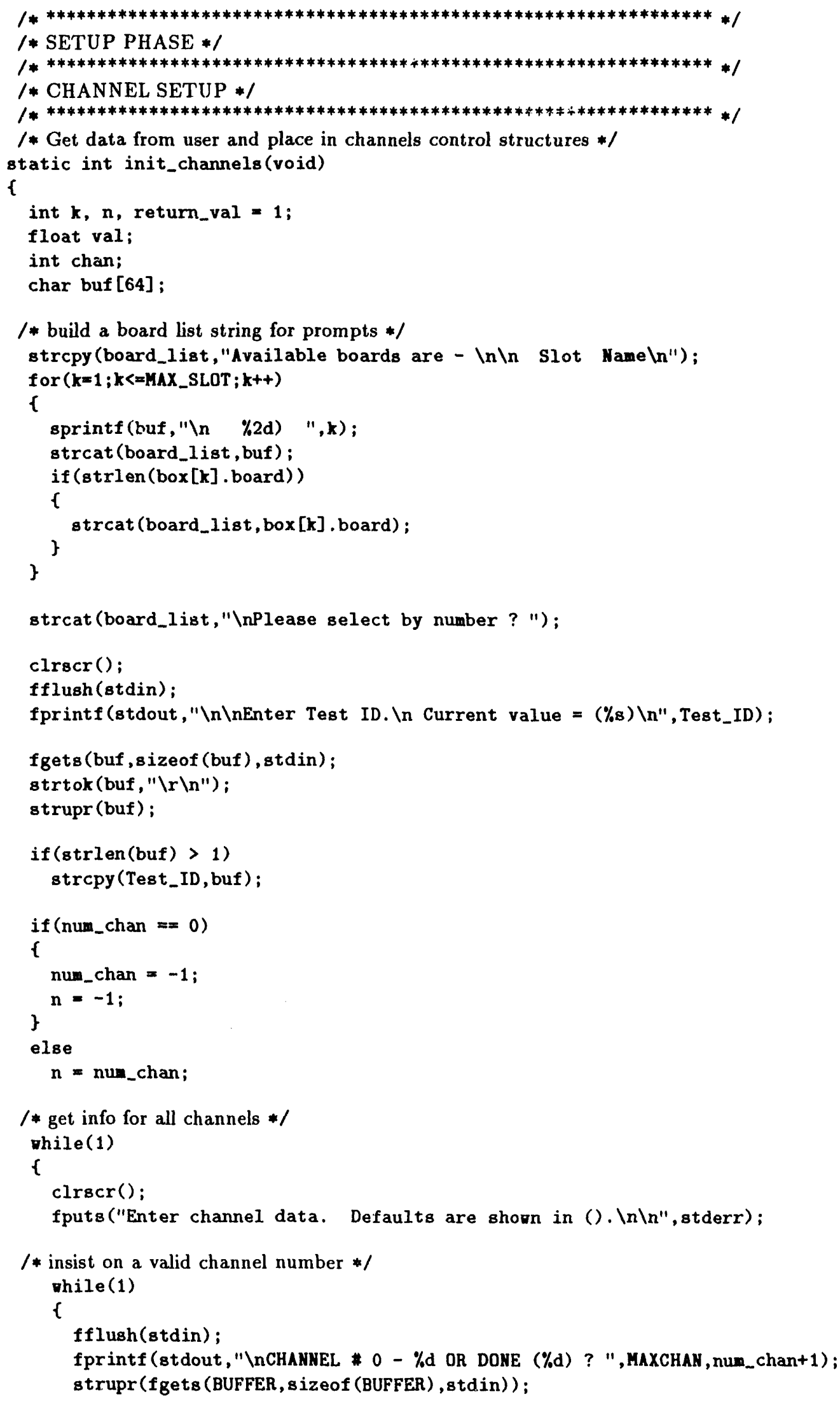




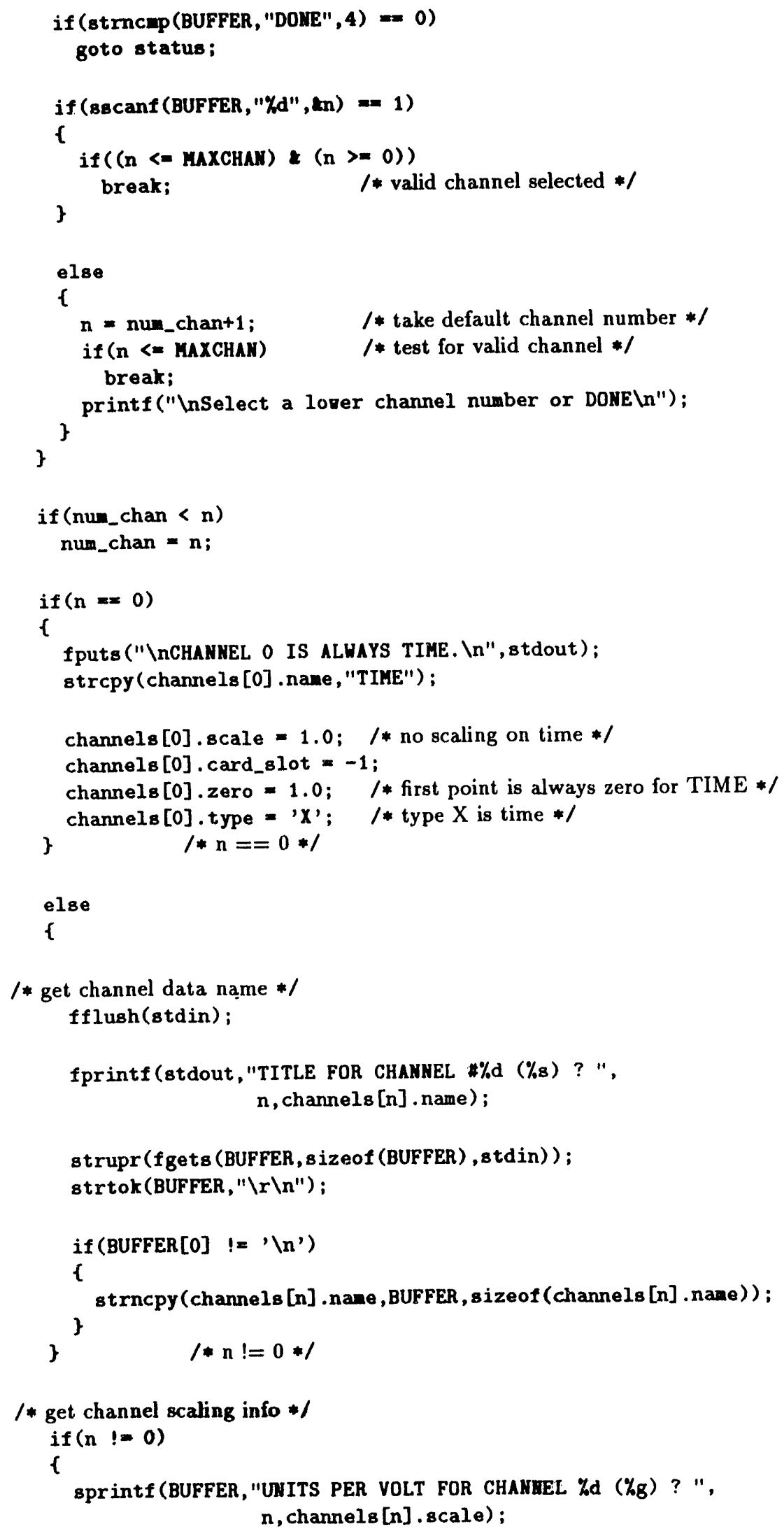




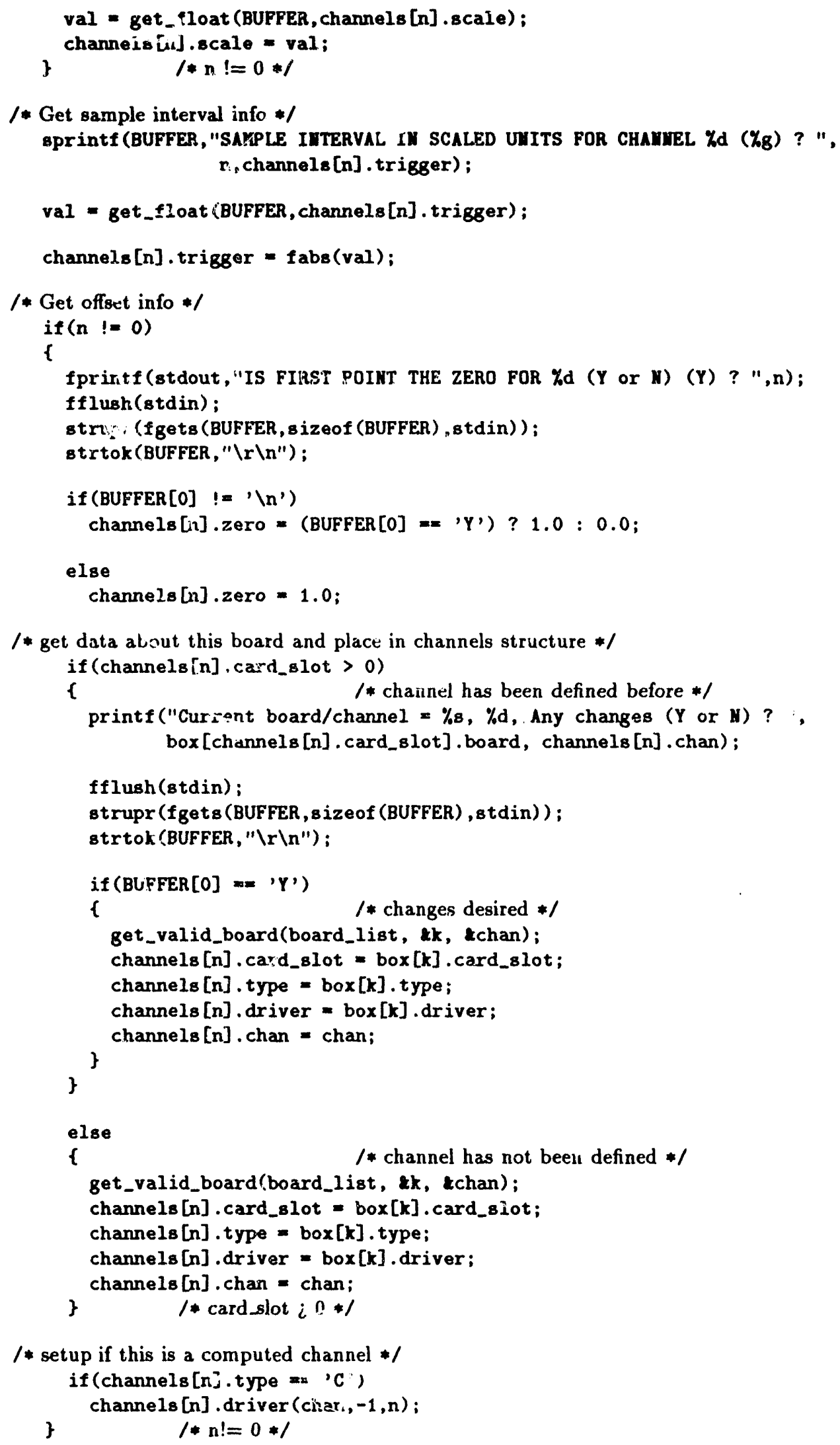




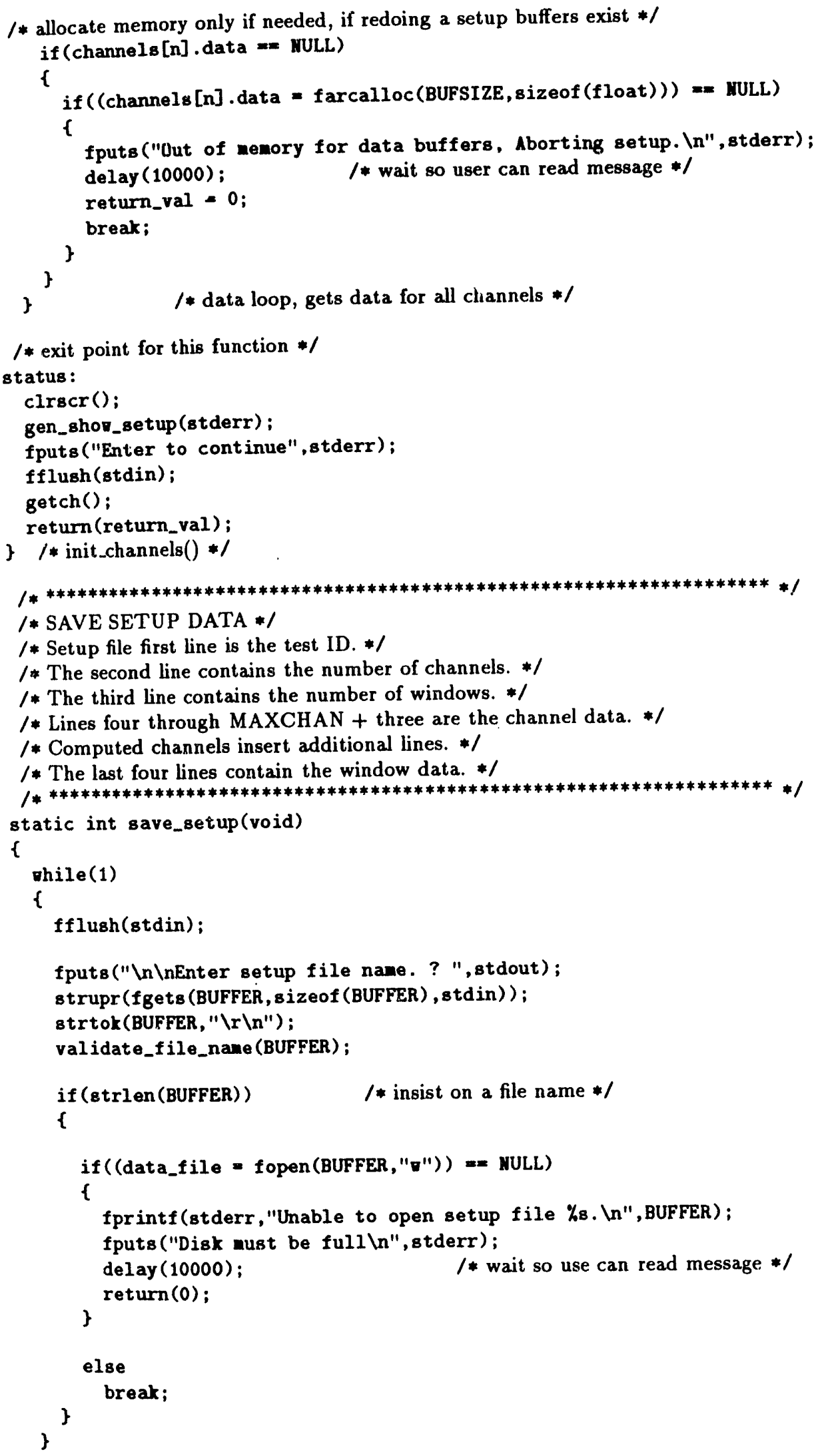






/* determine length of setup file so we do not read too much or little. */ $\mathbf{k}=0$; 


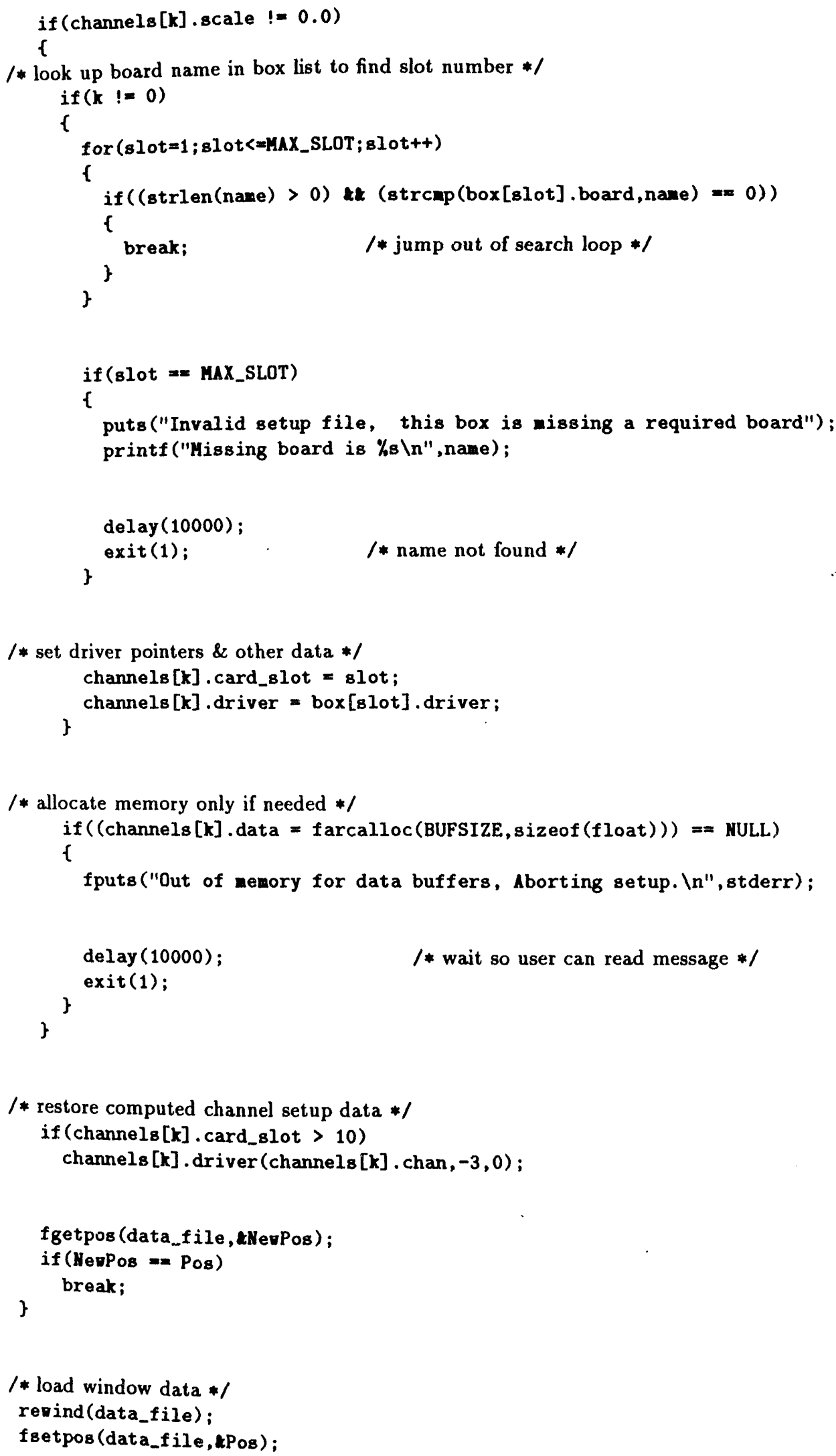




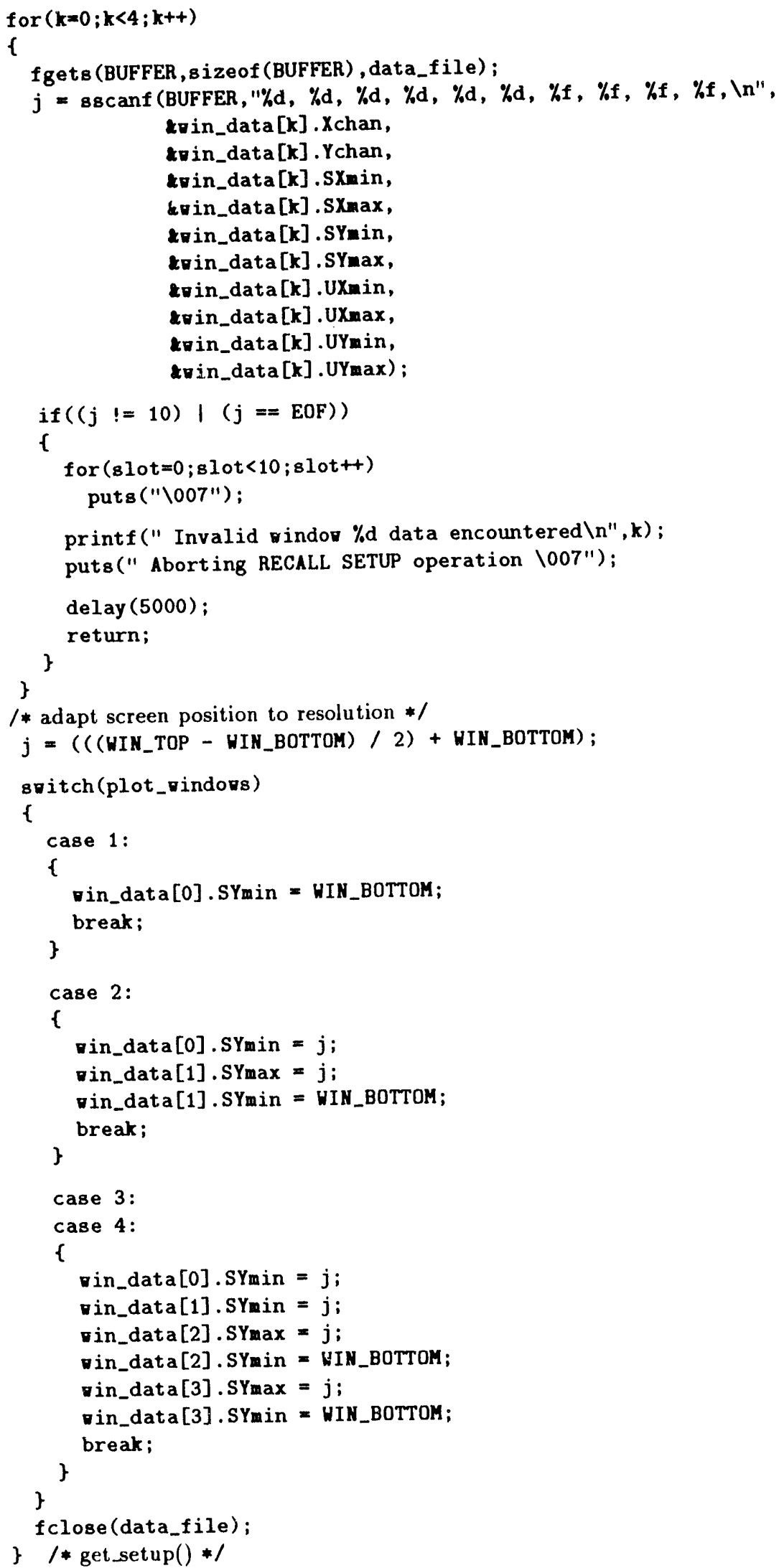









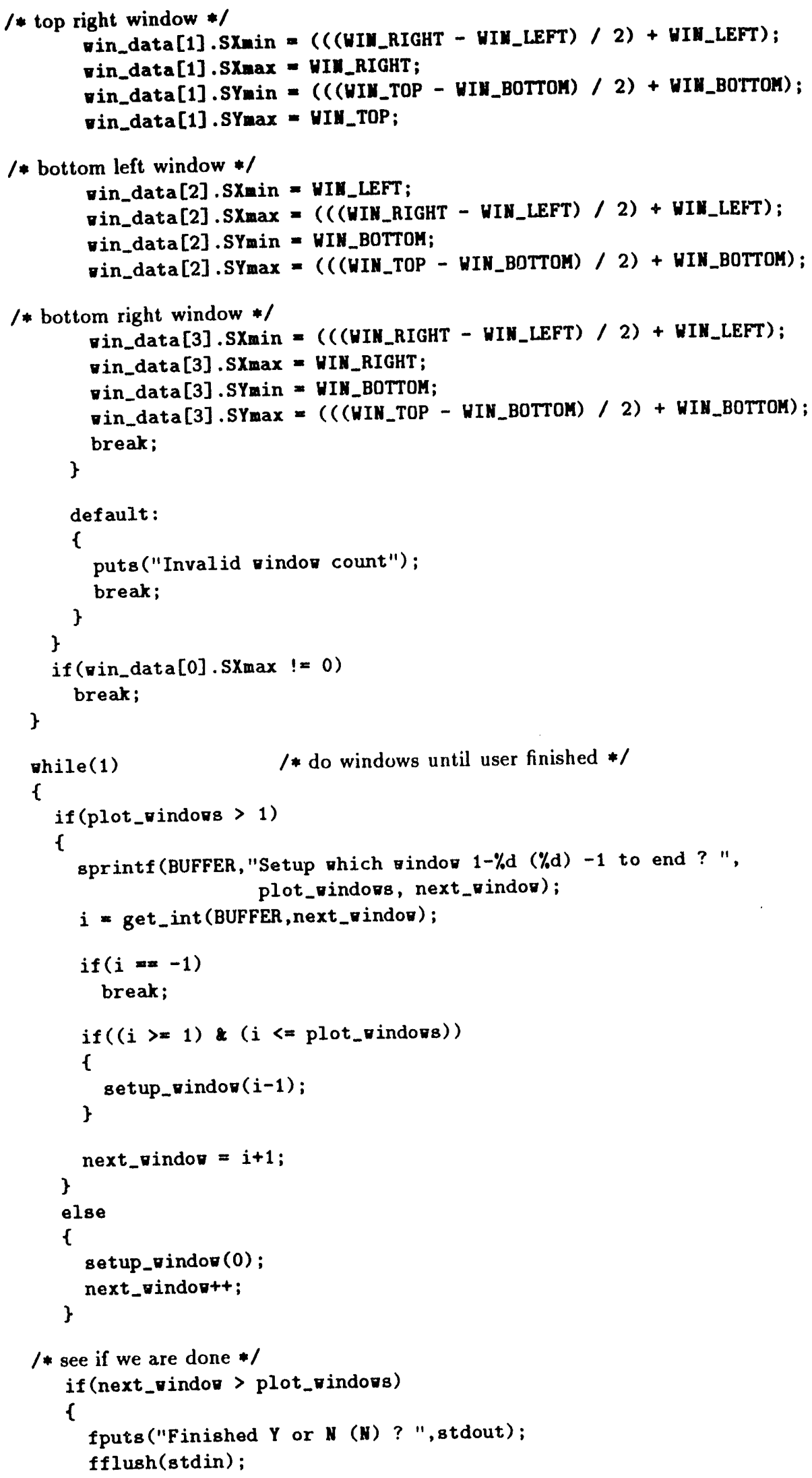




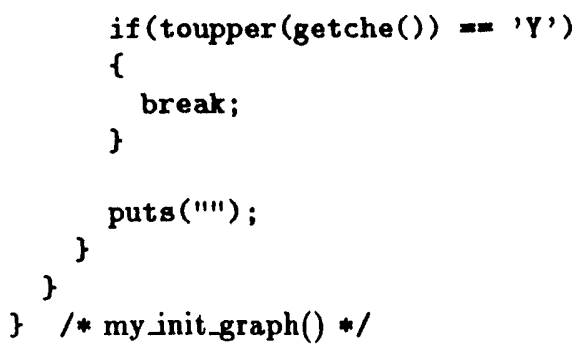

/* Get X-Axis channel for this window */ sprintf (BUFFER, "Enter channel to plot on vindor \%d X-Axis (\%d) or $(-1=$ none) ?", vindo +1 , $\nabla$ in_data[vindo $\nabla] . X(h a n)$;

chan = get_int (BUFFER, $\nabla$ in_data[ $[$ indo $\left.] . x_{\text {chan }}\right)$;

vin_data[vindov].Xchan $=$ chan;

if $($ chan $==-1)$

return;

/* Get Y-Axis channel for this window */ sprintf (BUFFER, "Enter channel to plot on vindop \%d Y-Axis (\%d) ?", windov+1, vin_data[vindov].Y(chan);

chan $=$ get_int (BUFFER, vin_data[ $[$ indo $] . Y$ chan) $;$ vin_data[rindor].Ychan = chan;

/* Get $X$-Axis minimum value to plot */ sprintf (BUFFER, "Enter X-Axis minimum value (\%g) ?", vin_data["indoø].UXmin);

min = get_float(BUFFER, vin_data[चindo $]$.UXmin);

1* Get $X$-Axis maximum value to plot */ sprintf (BUFFER,"Enter $X$-Axis maximum value $(\% g)$ ?", vin_data ["indow].UXmax);

$\max =$ get_float $(B U F F E R$, in_data[ $[$ indo $]$. UXmax $) ;$

/* store user data in proper order, swap if needed */ if $(\min <=\max )$

\{

vin_data["indo $]$.UXmin = min;

vin_data[vindor].UXmax $=\max$; \}

else

f

vin_data[ $[$ indor].UXmin $=\max$;

vin_data[rindo $].$ UXmax $=\min$; 
/* Get Y-Axis minimum value to plot */

sprintf (BUFFER, "Enter Y-Axis minimum value (\%g) ?", win_data[चindow].UYmin);

min = get_float (BUFFER, vin_data[ [indow].UYmin);

/* Get Y-Axis maximum value to plot *

sprintf (BUFFER, "Enter $Y$ - $A$ xis maximum value $\left(\%_{g}\right)$ ?", vin_data[चindov].UYmax);

$\max =$ get_float (BUFFER, $\nabla$ in_data[qindov].UYnax);

$/ *$ store user data in proper order, swap if needed */

if (min $<=\max$ )

f

vin_data["indoø].UYmin = min;

vin_data[rindor].UYmax $=\max$;

\}

else

\{

vin_data[vindo $]$ ] UYmin $=\max ;$

vin_data[ $[$ indo $]$.UYmax $=\min ;$

\}

\} /* setup_window ()$* 1$

$1 *$

/* PRINT CHAN DATA */

static void shor_setup(void)

\{

gen_shor_setup (printer);

fputc('\f',printer);

\} $/ *$ show_setup ()$* 1$

/******************************************************************/

$/ *$ general case show setup function $* /$

static void gen_shor_setup (FILE *dev)

\{

int $k$;

ftime(rstart_time);

/* get time stamp */

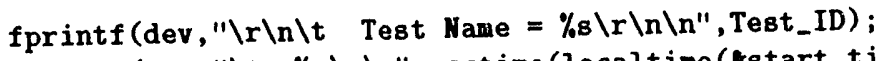

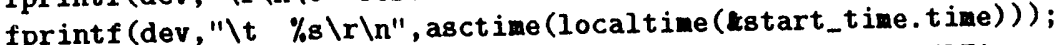

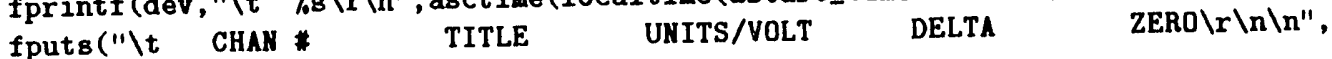
dev);

for $(k=0 ; k<=$ MAXCHAN; $k++)$

\{

if (channels $[\mathrm{k}]$. scale $!=0.0)$

\{

fprintf(dev," $\backslash$ t $\% 4 d \quad \% 17 s \quad \% 10.4 f \quad \% 10.4 f \quad \% d \backslash r \backslash n "$,

$k$, channels[k] name, channels[k].scale,

channels [k] .trigger, (int) channels [k] zero);

\}

\}

\} /* gen_show_setup */ 


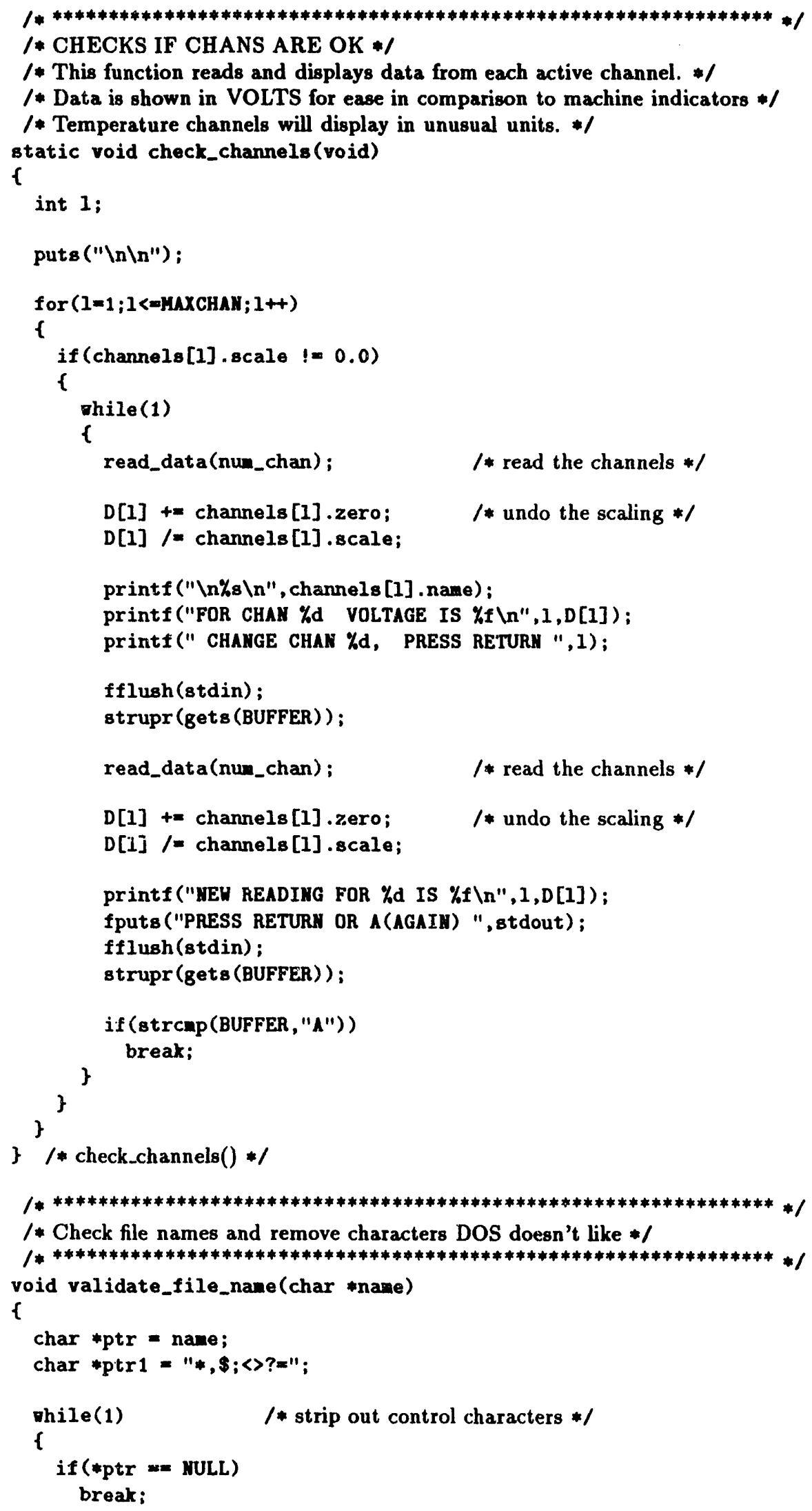




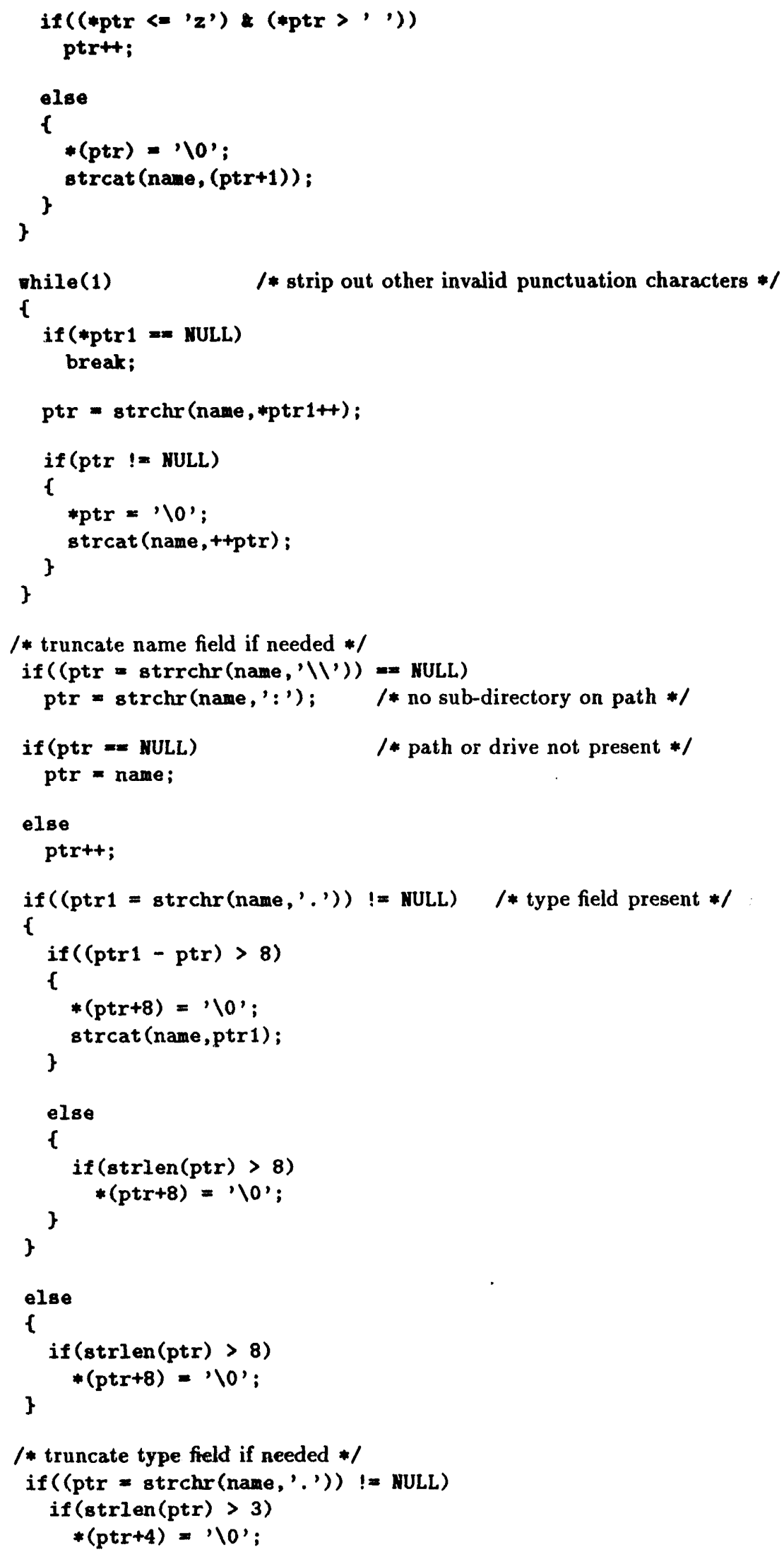


\} / * validate_file_name ()$* /$

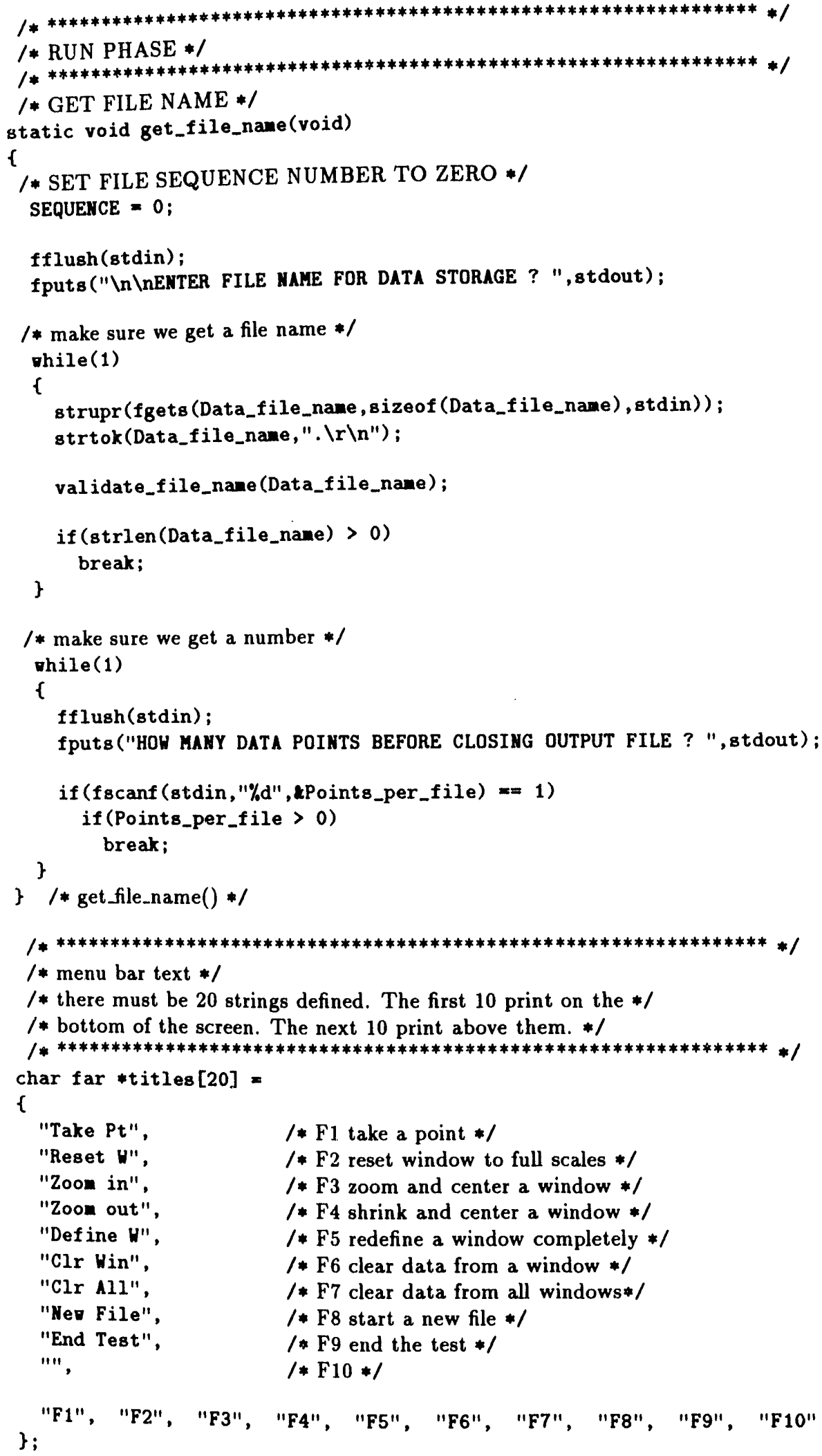




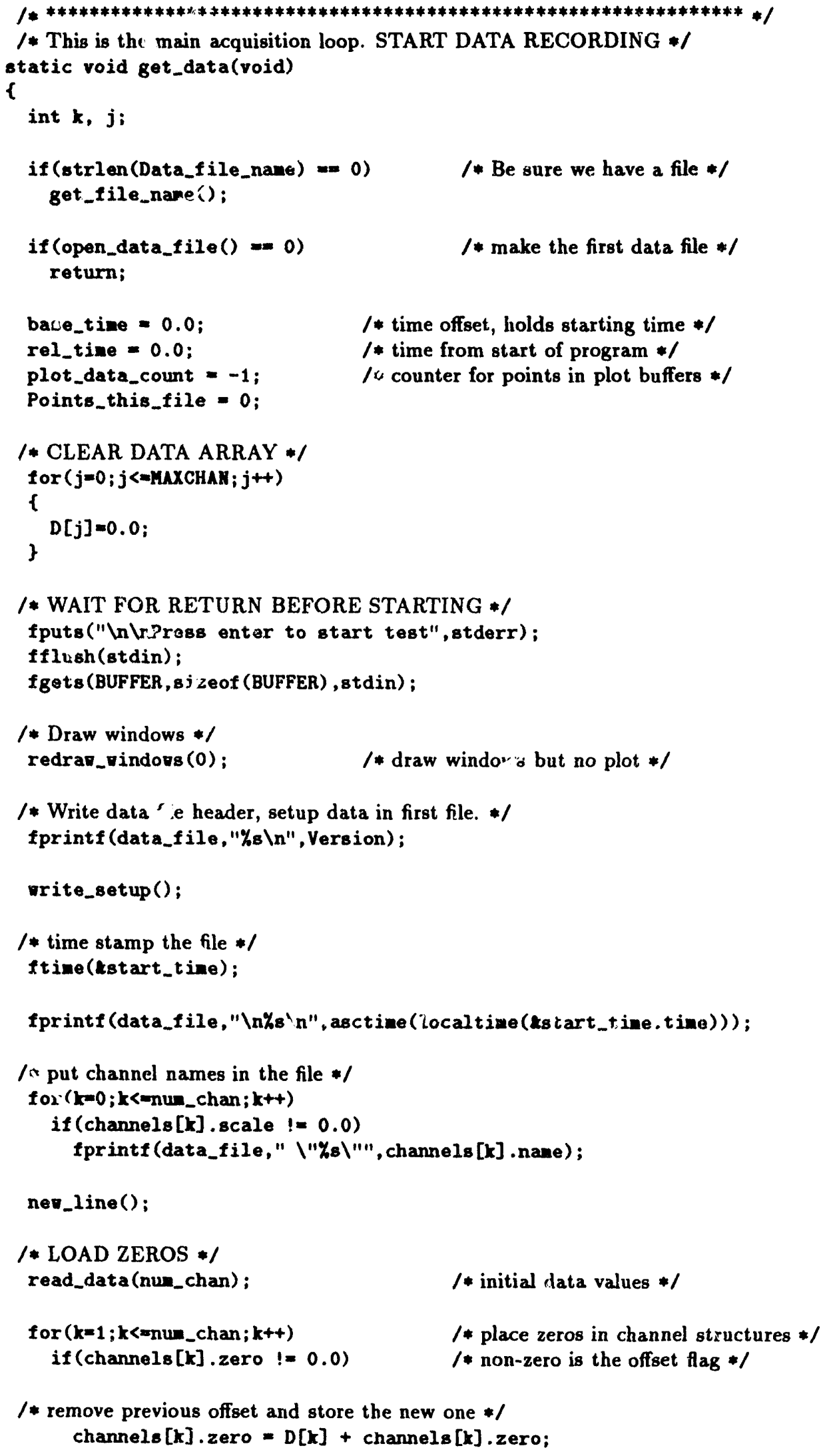




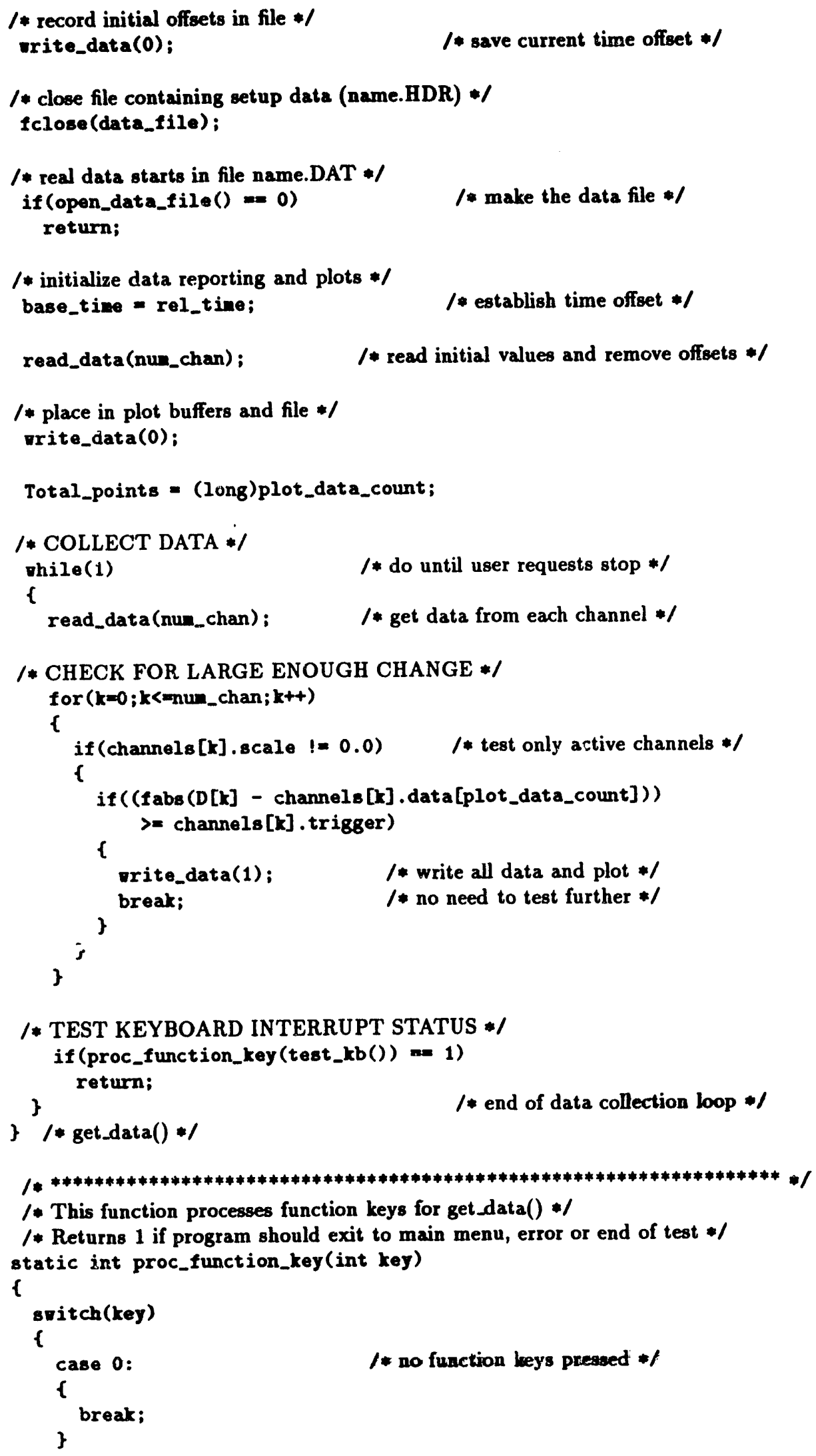




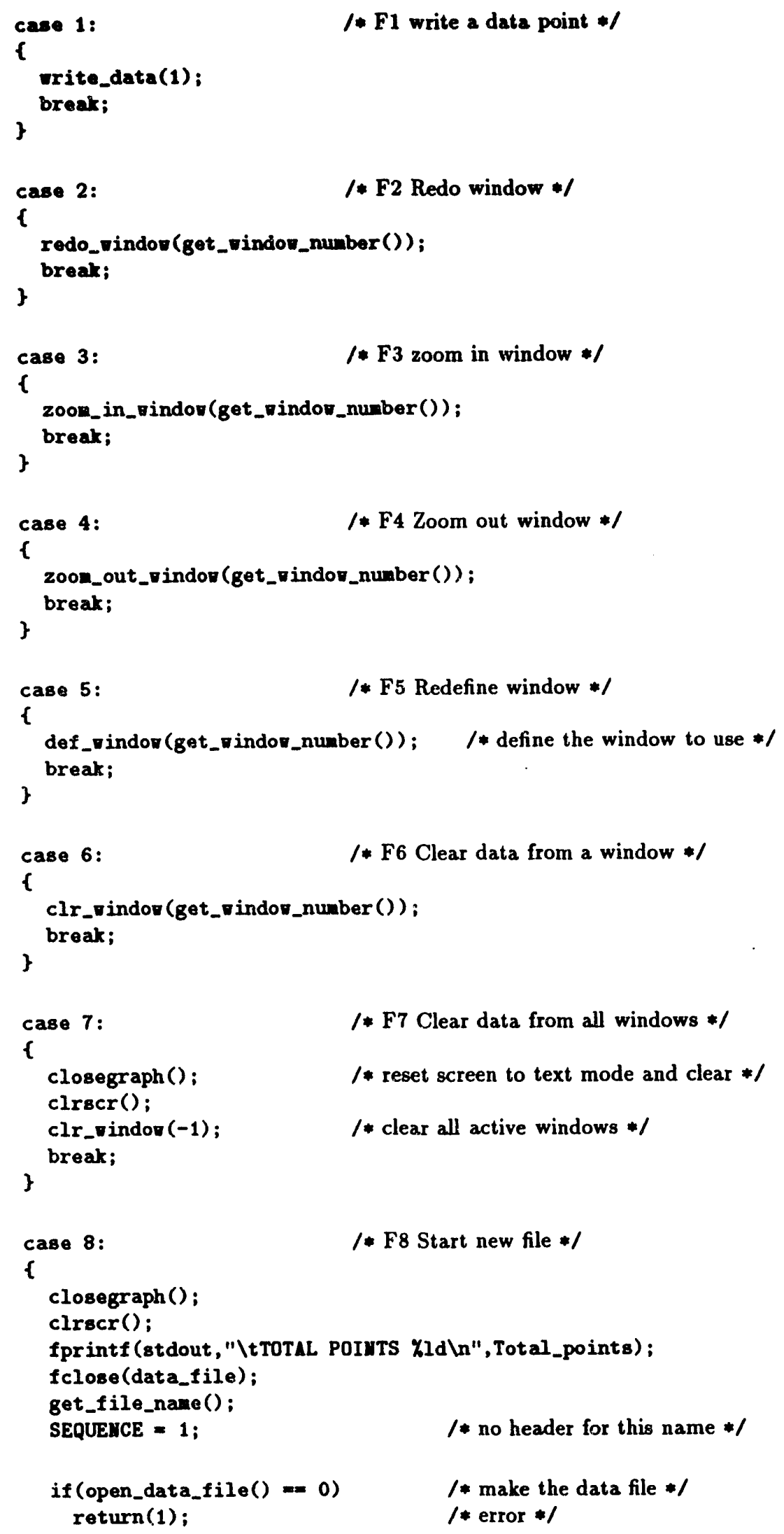




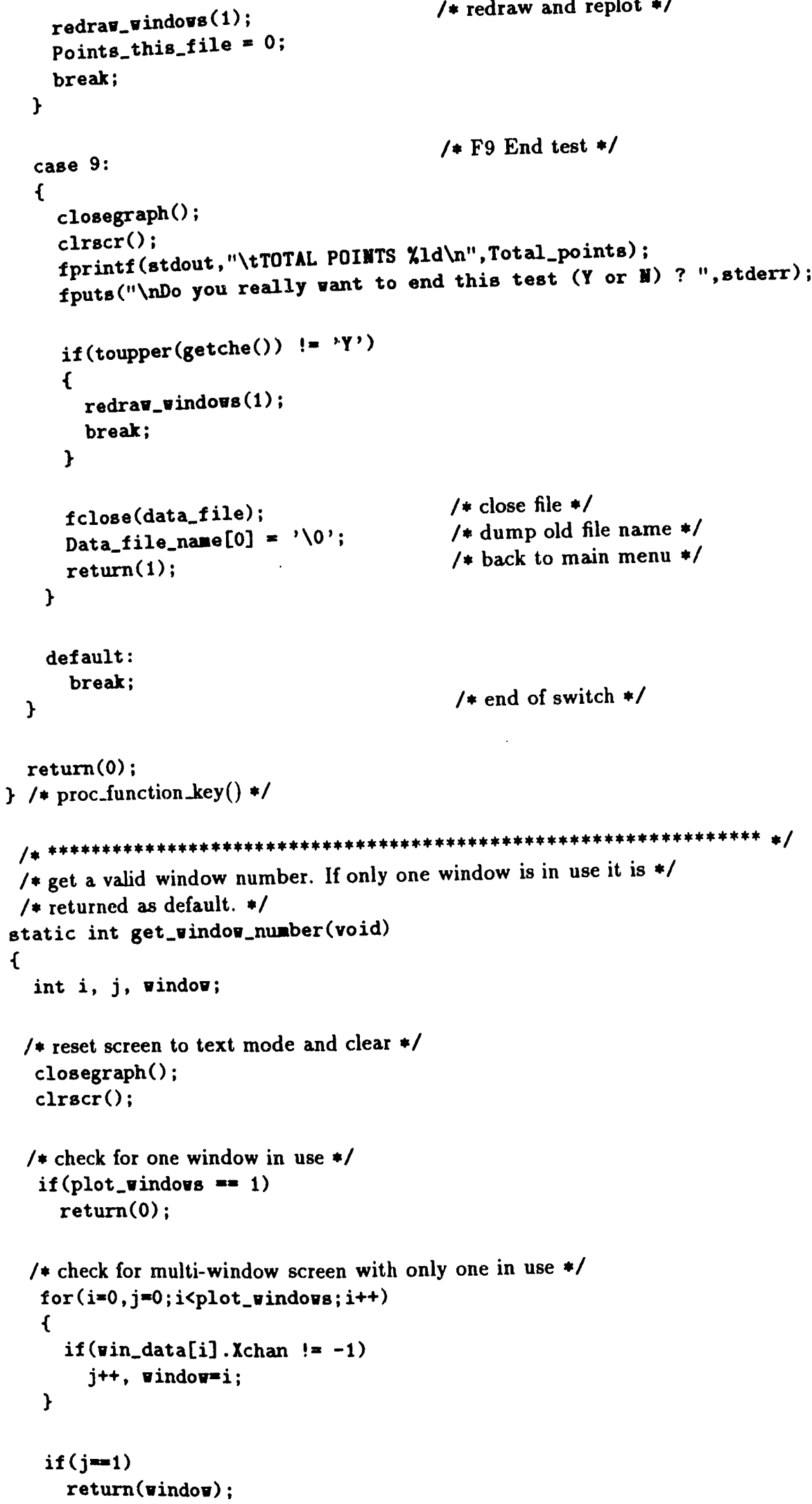


/* Multiple windows in use, get the desired one from user */

vhile(1)

t

fflush(stdin);

fprintf (stderr," Which vindow (1 - \%1d) ? "plot_vindovs);

if (scanf ("\%d", \&vindo $)=1$ )

if $((\text { indov }>=1) *(\text { vindov }<=\text { plot_vindovs }))^{-}$

break;

\}

return(--vindor);

\} $/ *$ get_window_number ()$* 1$

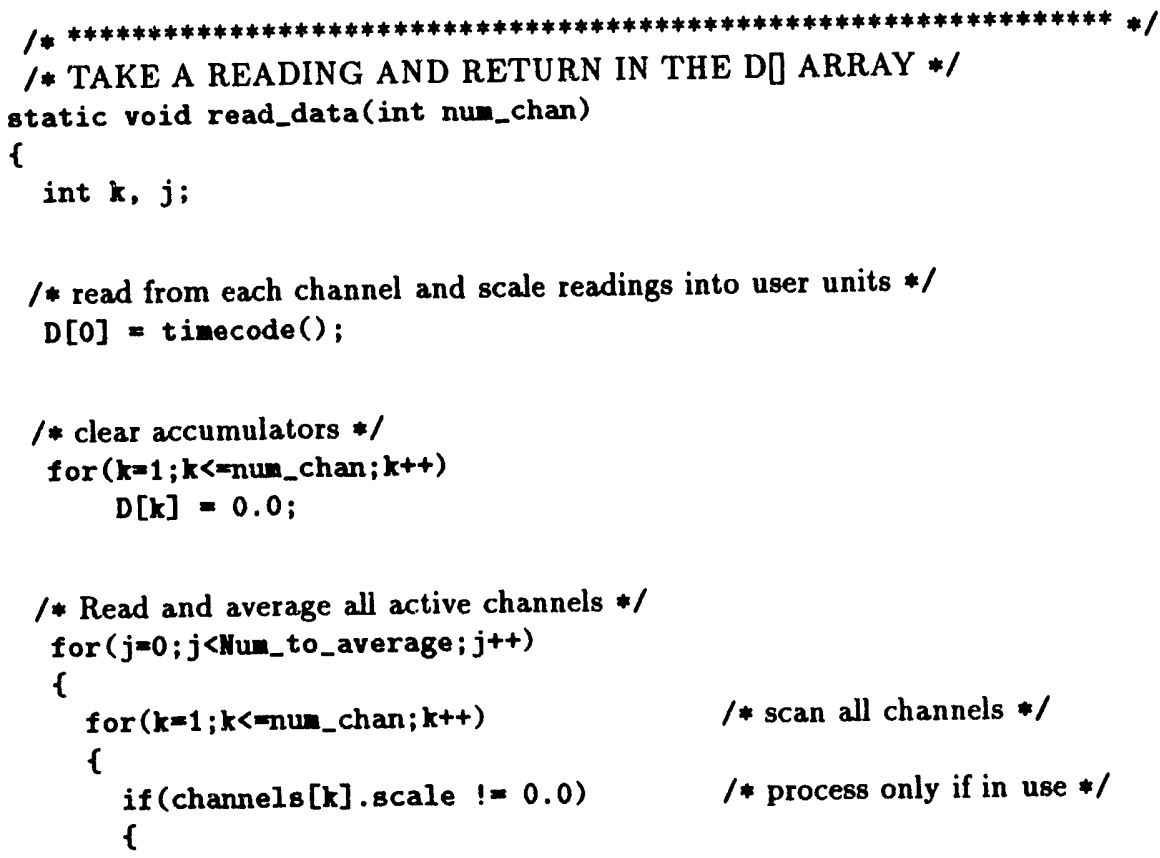

/* get raw reading in volts and divide by Num_to_average then accumulate */ $D[k]+=$ (channels $[k]$.driver (channels [k].chan,

\section{/ (float) Iun_to_average);}

channels [k] . card_slot, 1)

\}

子

\}

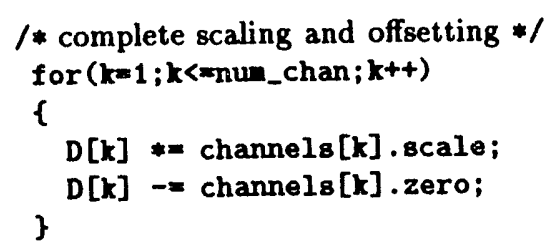

\} $/ *$ read_data ()$* f$ 


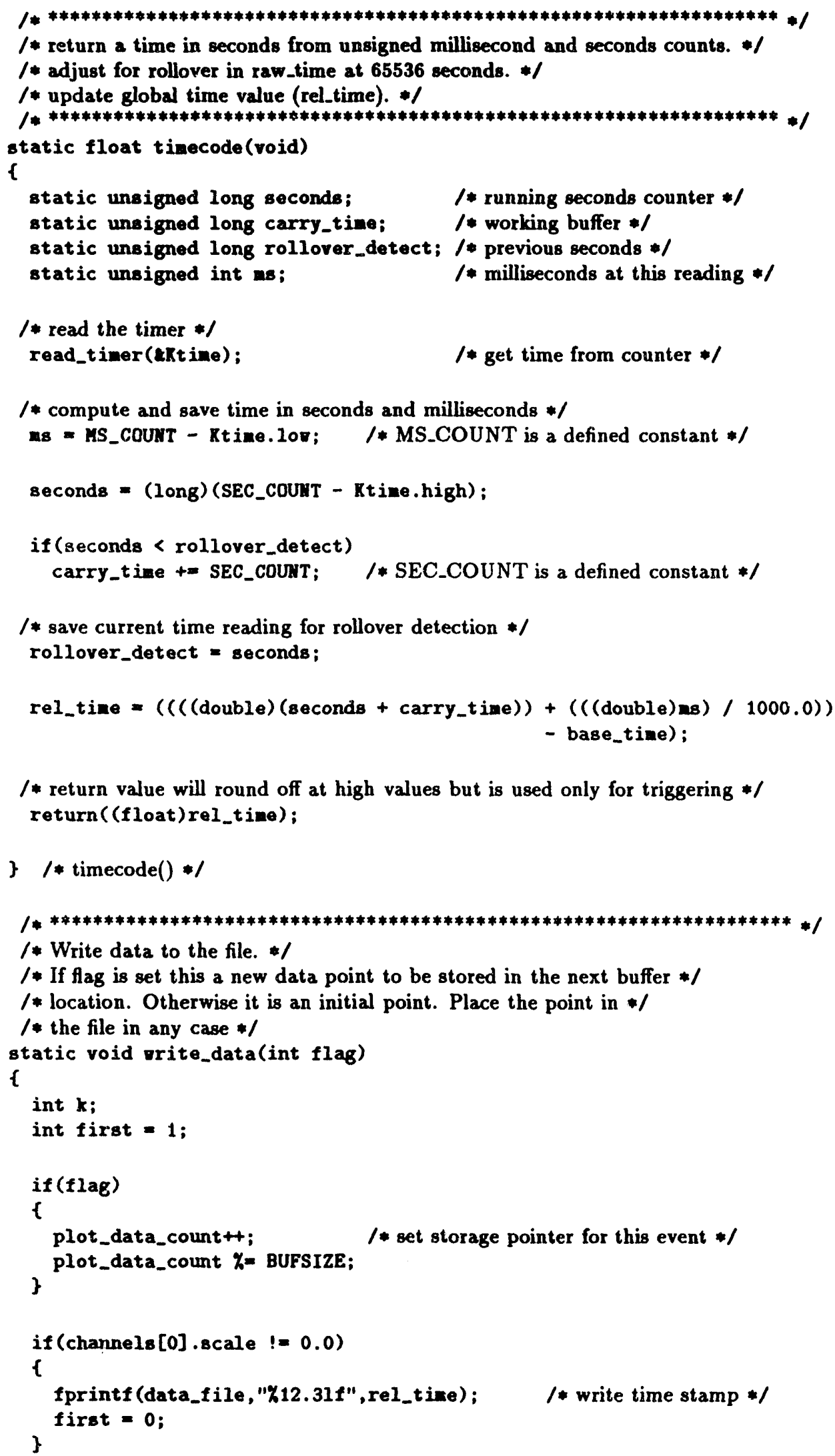




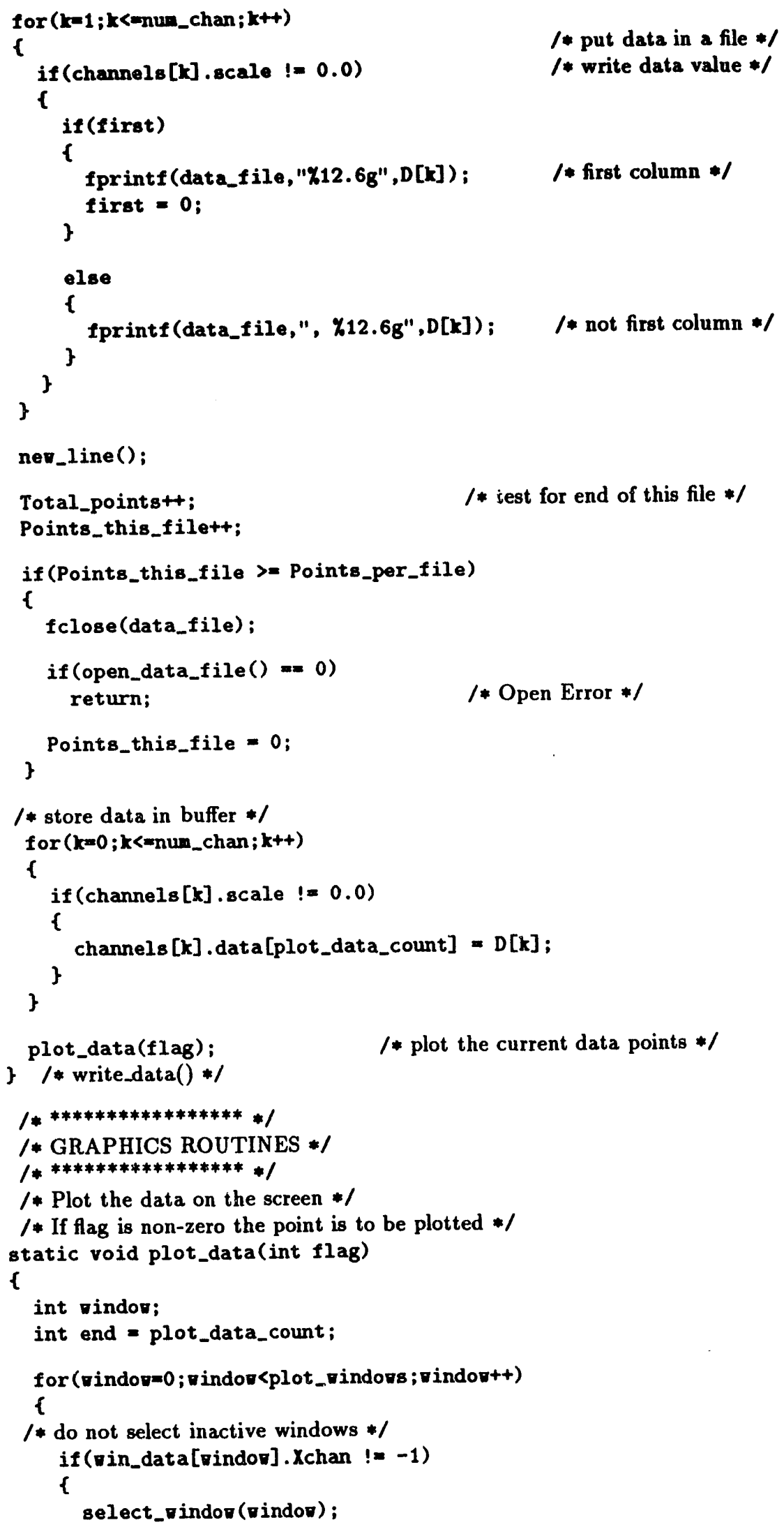

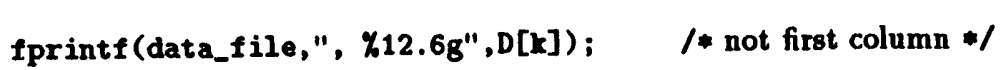

$/ *$ put data in a file */ /* write data value */ 


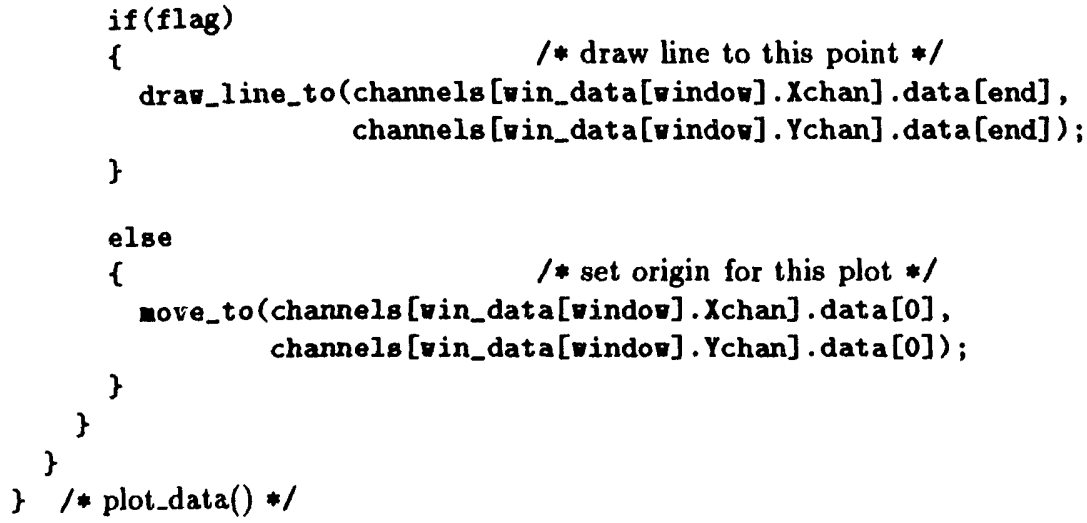

float $X, Y$;

/* absolute value of full scale for this channel */

$/ *$ scale is in units per volt. ${ }^{*} 10.0$ compensates for $10 \mathrm{~V}$ full scale $* /$

$1 *$ input range $* 1$

$X=f a b s$ (channels[vin_data[rindor].Xchan].scale $* 10.0$ )

$Y=f a b s\left(c h a n n e l s\left[\nabla i n \_d a t a[v i n d o r] . Y c h a n\right] . s c a l e * 10.0\right)$;

/* put new plot limits in window control structure */

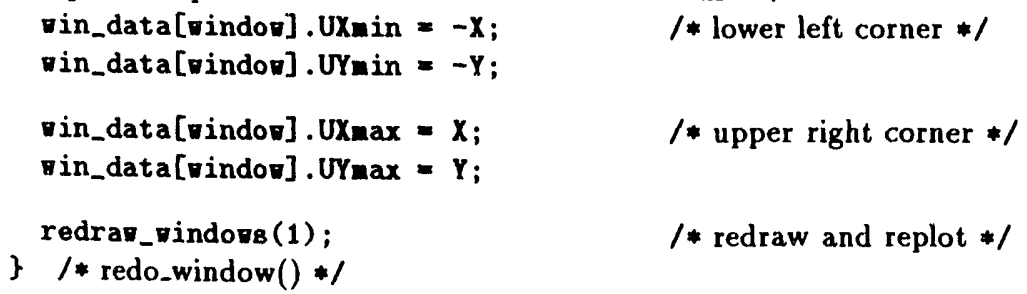


\} $1 *$ zoom_in_window ()$* 1$

$/ * * * * * * * * * * * * * * * * * * * * * * * * * * * * * * * * * * * * * * * * * * * * * * * * * * * * * * * * * * * * * * * * * * * * * 1$

/* Zoom window out. The window is demagnified by Zoom_Scale and the $*$ /

1* last data point is centered. The screen is completely redrawn $* 1$

$1 * * * * * * * * * * * * * * * * * * * * * * * * * * * * * * * * * * * * * * * * * * * * * * * * * * * * * * * * * * * * * * * * * 1$

static void zoom_out_vindov(int windor)

\{

zoom (window, $1.0 /$ Zoon_Scale);

redrav_vindors (1);

/* redraw and replot $* /$

\} /*zoom_out_window ()$* /$

$/ * * * * * * * * * * * * * * * * * * * * * * * * * * * * * * * * * * * * * * * * * * * * * * * * * * * * * * * * * * * * * * * * * * /$

/* This is a generic zoom function used by the above functions $*$ /

$1 * * * * * * * * * * * * * * * * * * * * * * * * * * * * * * * * * * * * * * * * * * * * * * * * * * * * * * * * * * * * *)$

static void zoom (int vindor, float scale)

\{

float $x$, deltax, $y$, deltay;

if (rin_data[ $[$ indo $]$ ].Xchan $!=-1$ ) /* only zoom active windows */

\{

/* Get last data point */

$x=$ channels [win_data[rindow].Xchan].data[plot_data_count];

y z channels[चin_data[चindov].Ychan].data[plot_data_count];

1* compute offsets, (range*scale), then divide by 2 . This provides */

$1 *$ offsets each side of the last point *I

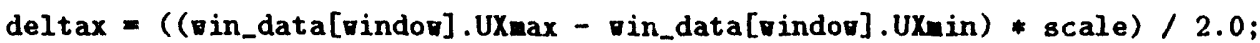

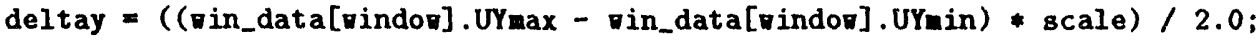

/* put new plot limits in window control structure */

vin_data[rindor].UXnin $=x-$ deltax;

$1 *$ lower left corner */

vin_data[вindo $]$.UYain $=y-$ deltay;

/* upper right corner $* /$

3

vin_data[rindog].UXmax $=x+\operatorname{deltax}$

\} $/ * \operatorname{zoom}() * 1$

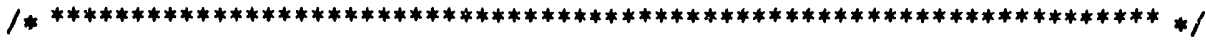

1* Clear data from a window. All data is erased from the specified */

/* window by replacing it with the last data value. The last point */

/* remains to initialize the plot. If the window number is -1 all */

/* windows will be cleared. */

static void clr_pindo (int vindov)

f

int $i, k, x, y$;

float $X, Y$;

if (

f

for $(i=0 ; i<\operatorname{MAXHIKDOWS;i++)}$

\{

if ( in_data $[i]$.Xchan $!=-1) \quad /$ Active windows only */

\{

$x=$ win_data[i].Xchan;

$y=\nabla$ in_data $[i] . Y$ chan; 


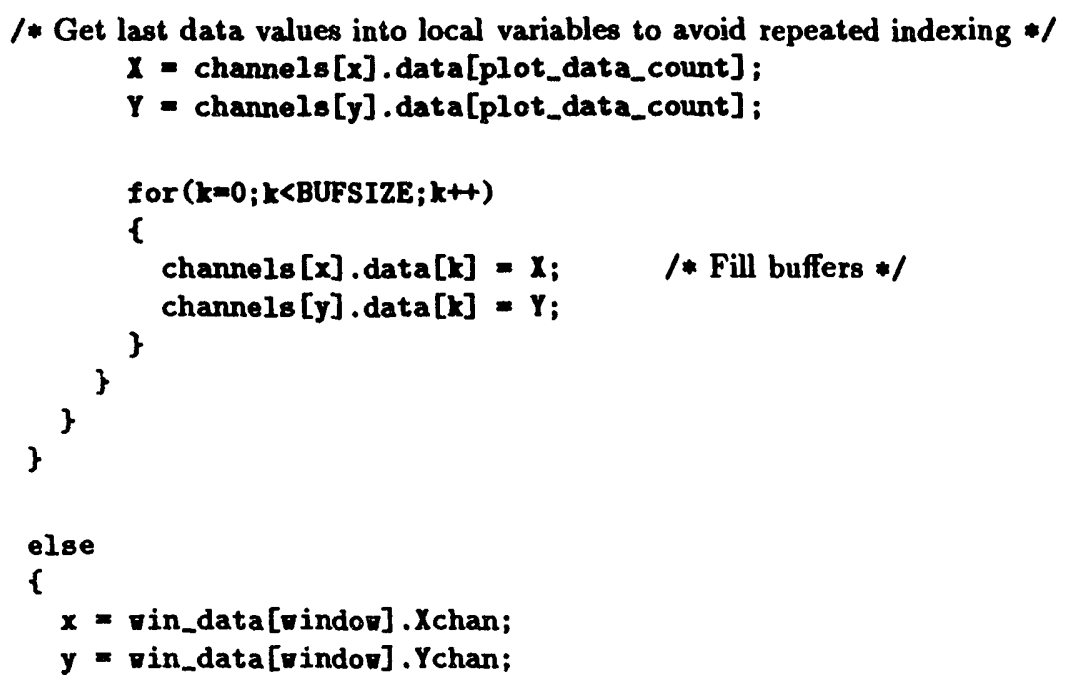

/* Get last data values into local variables to avoid repeated indexing */

$X=$ channels $[x]$.data[plot_data_count];

$Y=$ channels $[y]$.data[plot_data_count $]$;

for $(k=0 ; k<B U F S I Z E ; k++)$

f

channels $[x]$.data $[k]=X ; \quad / *$ Fill buffers $* /$ 


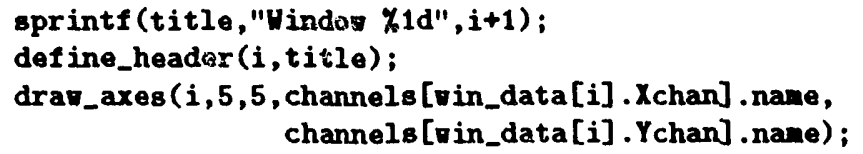

/* do the plot with wrap around until the latest point is encountered */ $\mathrm{j}=1$; /* default for one data point */

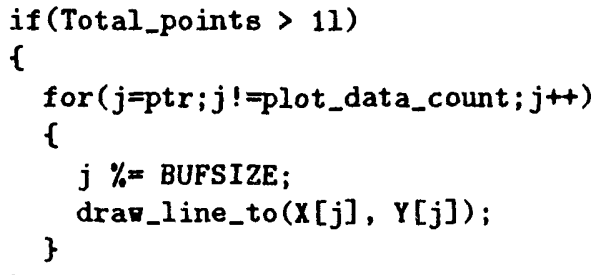




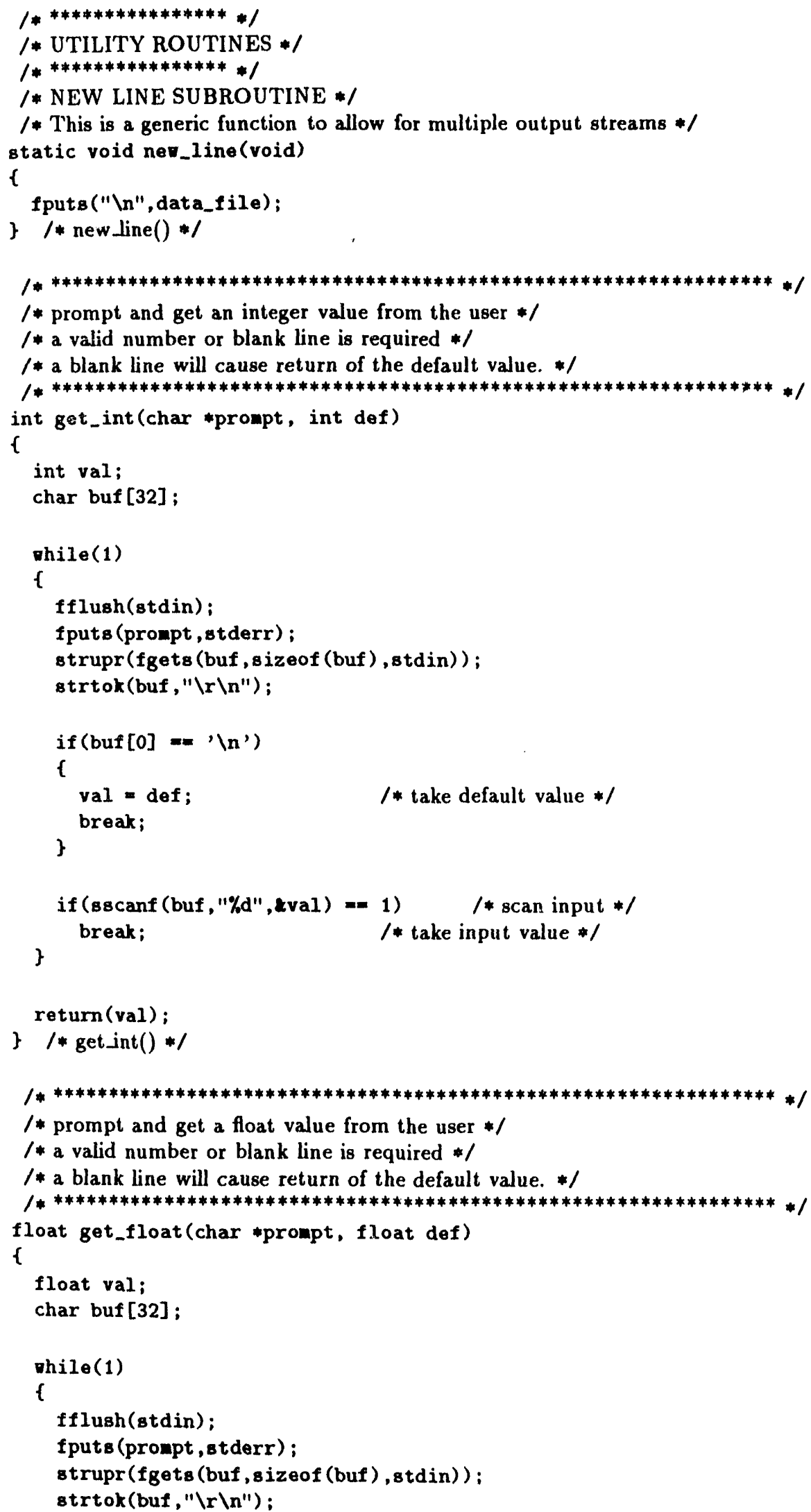




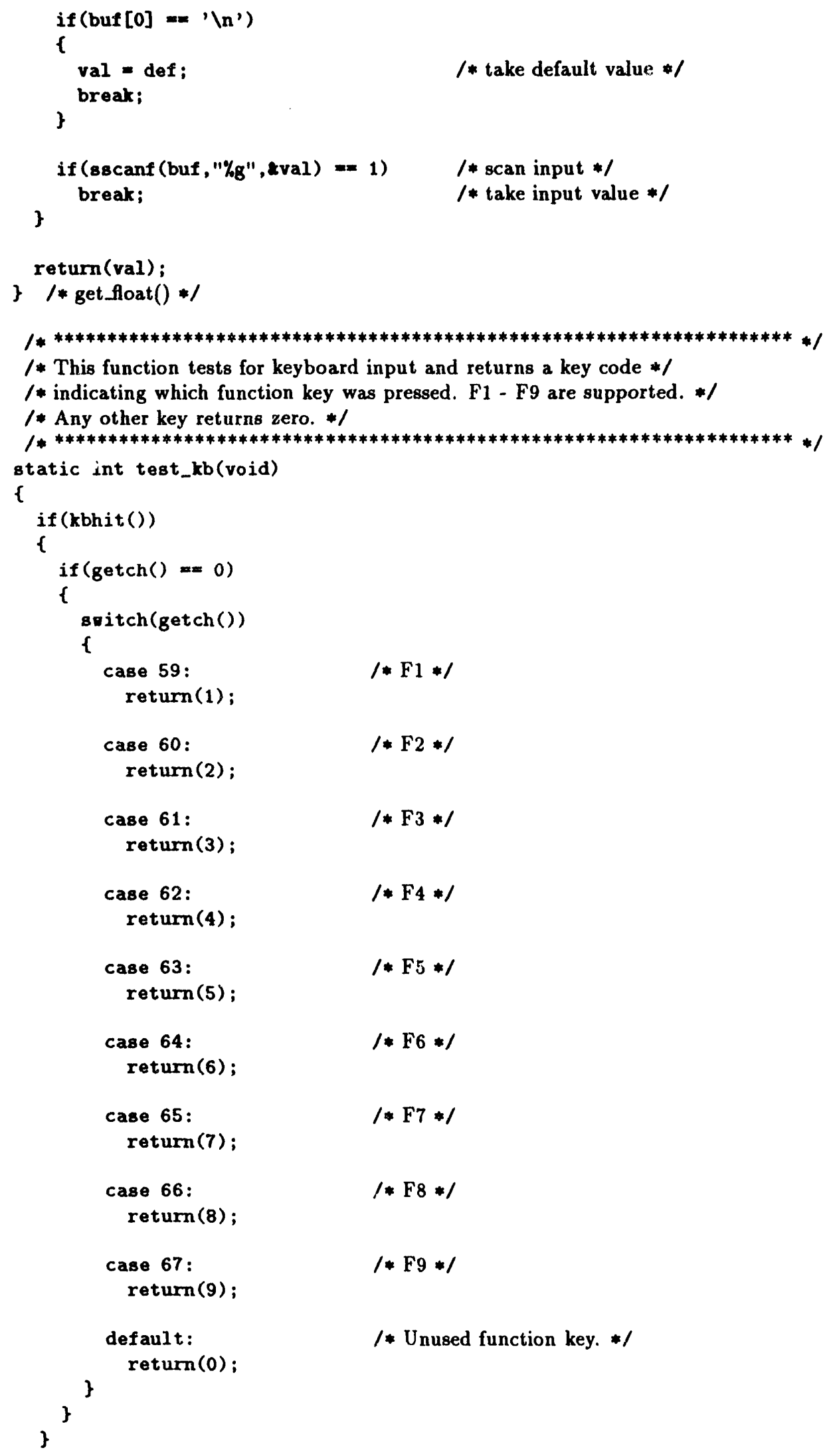




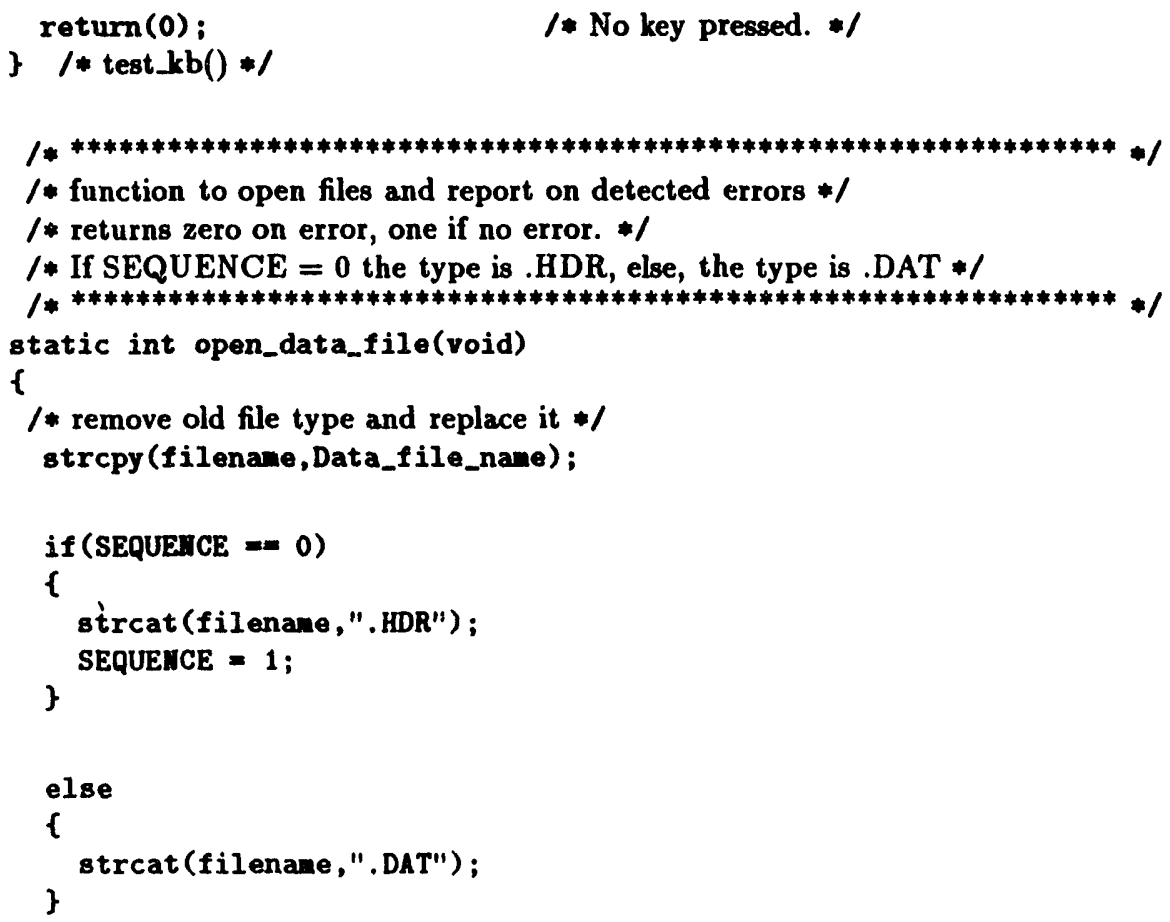

/* Open in append mode so we do not destroy data if the file exists */

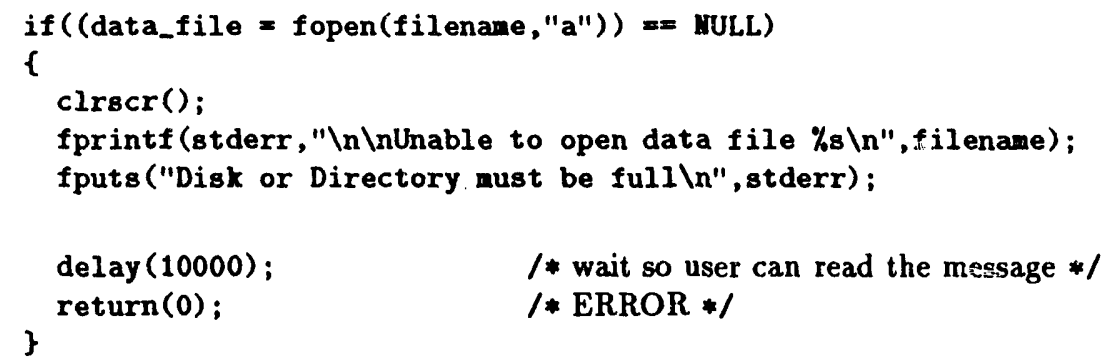




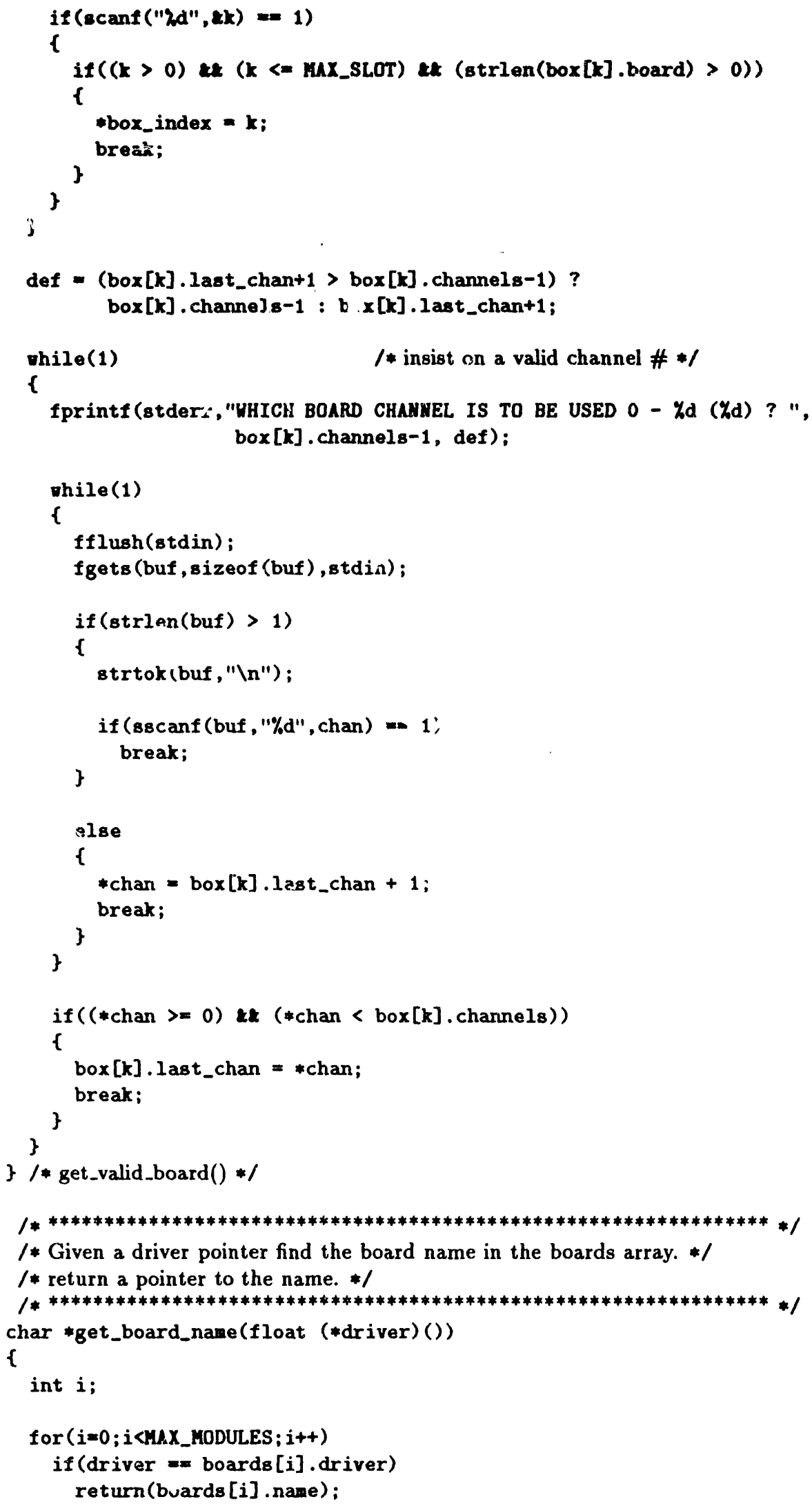


return (NULL);

? $/$ * get_board_name ()$* 1$

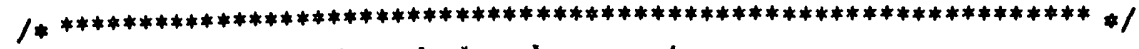

/* find driver pointer given the board name $*$ /

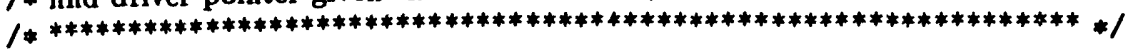

float (*find_driver_pointer (char *name)) ()

f

int $i$;

for $(i=0 ; i<$ HAX_HODULES; $i++)$

if (strncup (nane, boards [i] name, 8) $=0$ )

return(boards [i].driver);

return (MULL);

f / * find_driver_pointer ()$* /$ 


\section{B .1 TEMP.C - Temperature Channel Driver}

$/ * * * * * * * * * * * * * * * * * * * * * * * * * * * * * * * * * * * * * * * * * * * * * * * * * * * * * * * * * * * * * * * * * * * * * 1$

/* TEMP.C */

/* This module implements thermocouple temperature readings for */

/* DATAVG. It is assumed that a Keithley AIM7 is present. */

/* This pseudo-board supports 16 channels. */

$1 * * 1$

$/ * * * * * * * * * * * * * * * * * * * * * * * * * * * * * * * * * * * * * * * * * * * * * * * * * * * * * * * * * * * * * * * * * * * * *)$

$/ *$ Written by $* /$

$/ *$ R. D. Hardy */

/* Sandia National Laboratories */

/ Department $6117 * /$

/* April 17, $1992 * /$

$1 * * 1$

/* Comments configured for C2LATEX and LTT $\mathrm{XX}$

$/ * * * * * * * * * * * * * * * * * * * * * * * * * * * * * * * * * * * * * * * * * * * * * * * * * * * * * * * * * * * * * * * * * * * * * 1$

$/ *$ On entry if card_slot $==-1$ call the initialization code to setup $* /$

/ * local scaling data and a list of the physical channels to be used. */

$/ *$ If card_slot $==-2$ call the channel data save function. */

/* If card_slot $==-3$ call the channel restore function. */

/* If card $\_$slot $i=0$ compute the temperature in degrees $\mathrm{C}$. $* 1$

$/ * * * * * * * * * * * * * * * * * * * * * * * * * * * * * * * * * * * * * * * * * * * * * * * * * * * * * * * * * * * * * * * * * * * * * 1$

/ MODIFICATION HISTORY */

/* March 25, $1993 * /$

/* TEMP.CFG must be in the DATAVG directory. RDH. */

$/ * * 1$

$/ *$ June $25,1993 * /$

/* Made extensive changes in init(), restore() and build_tables(). */

$/ *$ This module did not properly initialize itself. RDH */

$/ * * 1$

$1 * * 1$

$/ * * * * * * * * * * * * * * * * * * * * * * * * * * * * * * * * * * * * * * * * * * * * * * * * * * * * * * * * * * * * * * * * * * * * * 1$

\#include "datavg.h"

*define MAX_TC_TAB 5

/* number of thermocouple types $* /$

/ function prototypes $* /$

static void init (int chan);

static int find_board(void);

static void init_error (char *text);

static void save(int chan);

static void restore(int chan);

static struct spline_tab oget_TC_type(void);

static float scale_temp(float TEMP, struct spline_tab $*$ TTAB, int card_slot);

static roid build_tables (roid);

static int $f l a g=0$;

typedef struct spline_tab

\{

double *xa; $\quad 1 *$ Pointer to temp. array *

double *ya;

double *y2a;

int $n$;

$/$ * Pointer to voltage array */

/* Pointer to coefficient array */

char type;

/ Number of elements in the arrays $* /$

f:

/ Thermocouple type character $*$ /

static struct spline_tab TC[KAX_TC_TAB]; 
/* array of structures holding channel data */ static struct

t

int chan; $\quad / *$ real board channel $* /$

struct spline_tab *table; $\quad /$ * pointer to spline table for this type */

\} chanl [16];

int slot;

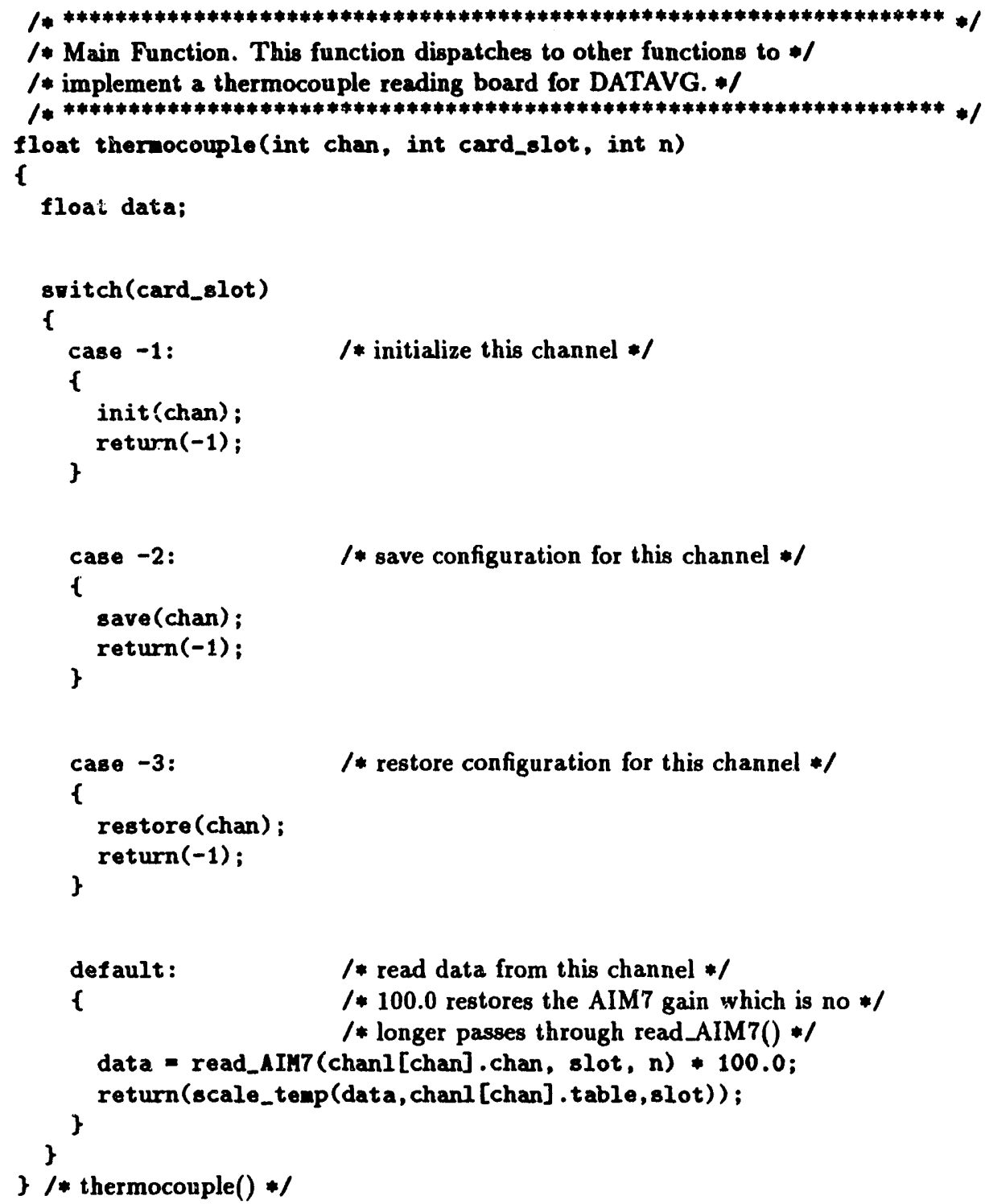


1* Initialize the thermocouple spline tables if not already done */

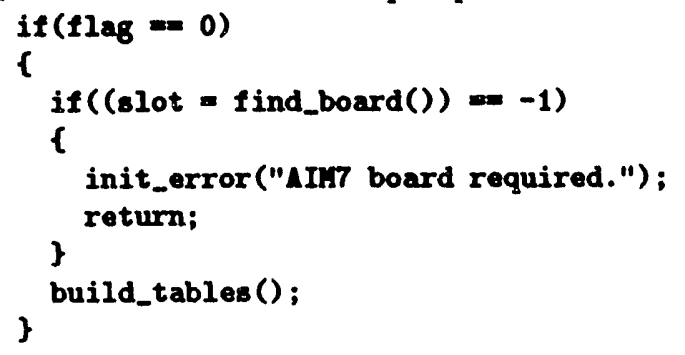

/* get the thermocouple type $* /$

chanl[chan] .table = get_TC_type();

f $/$ init ()$* 1$

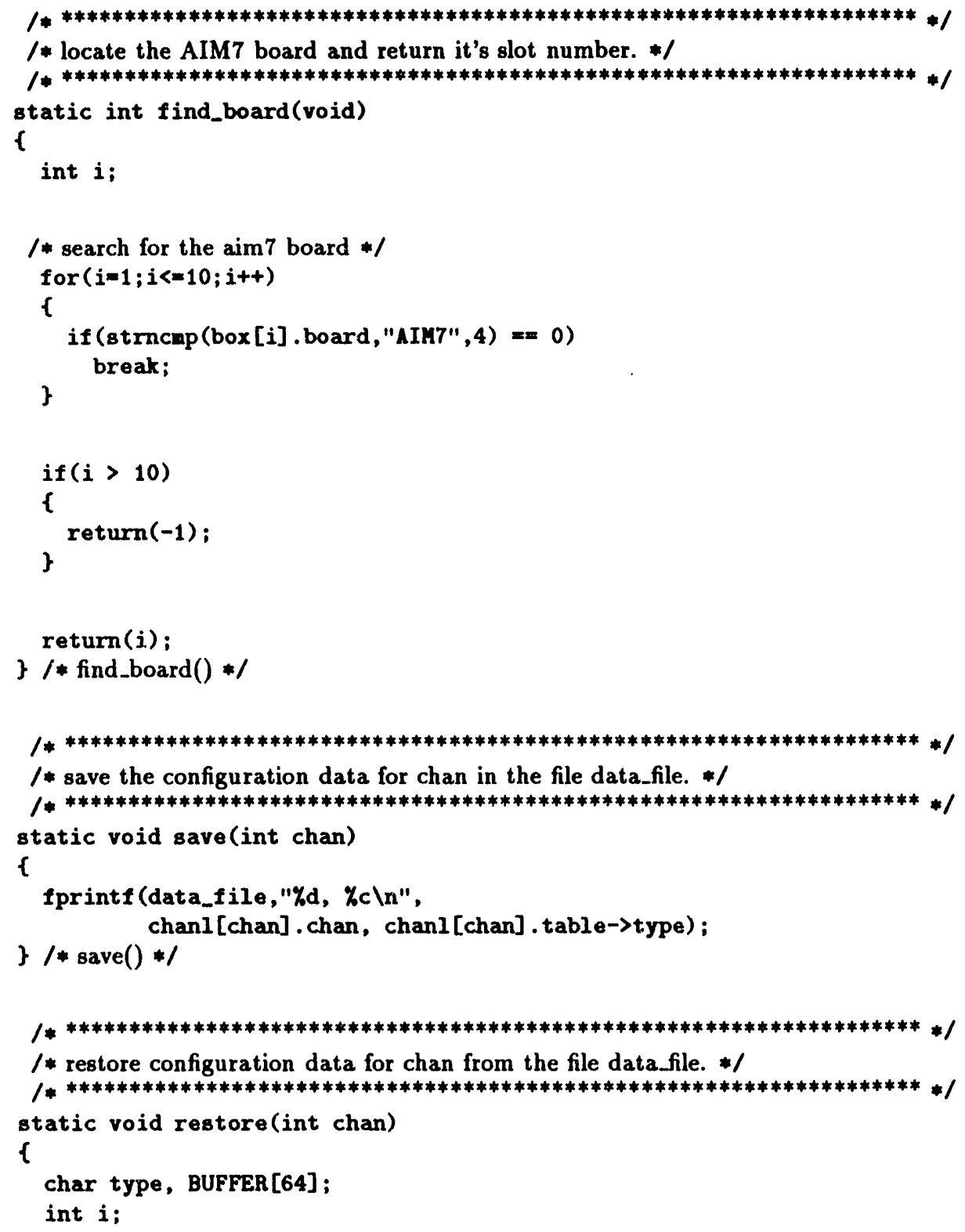




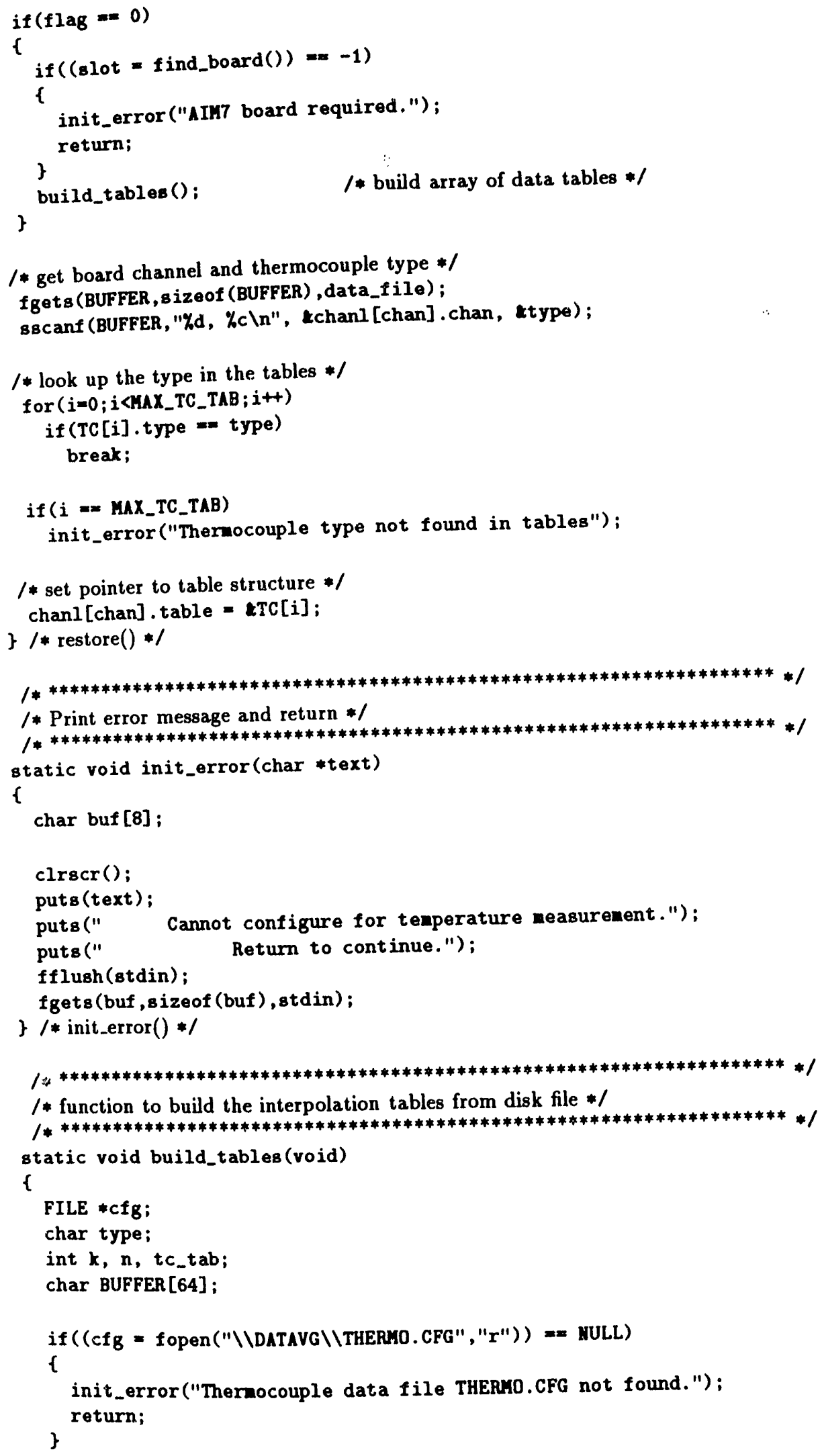




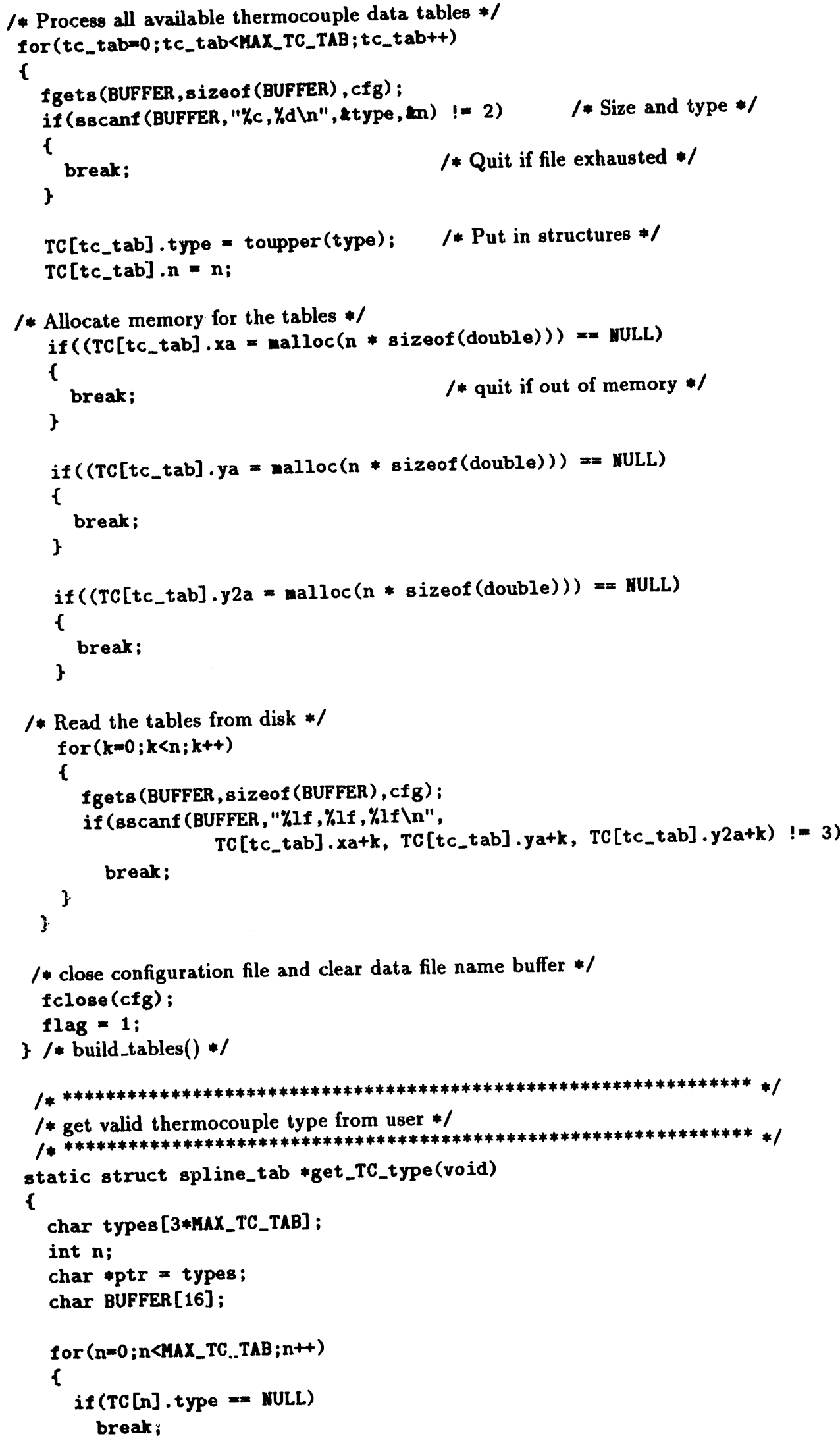




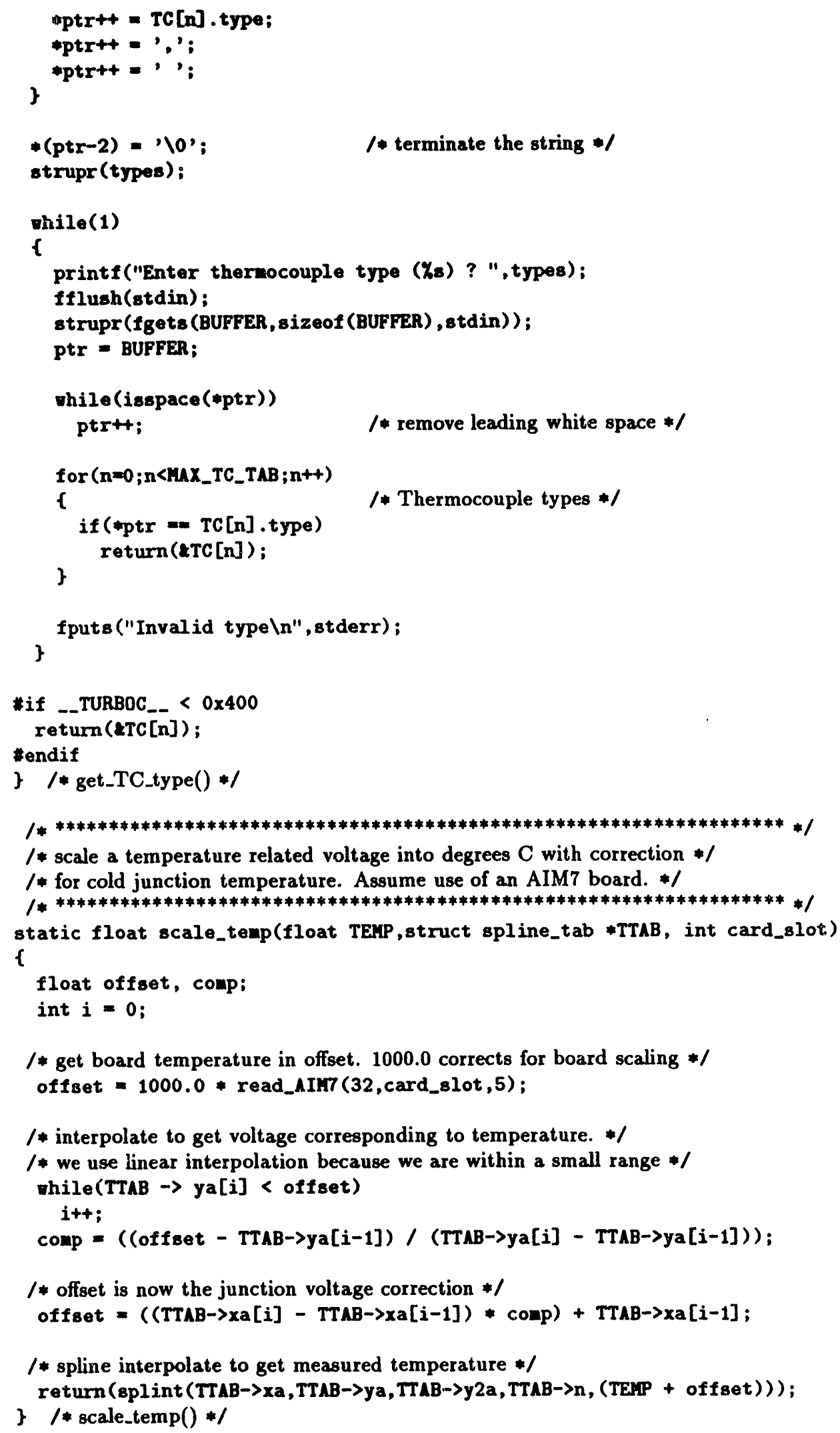




\section{500LIB.H}

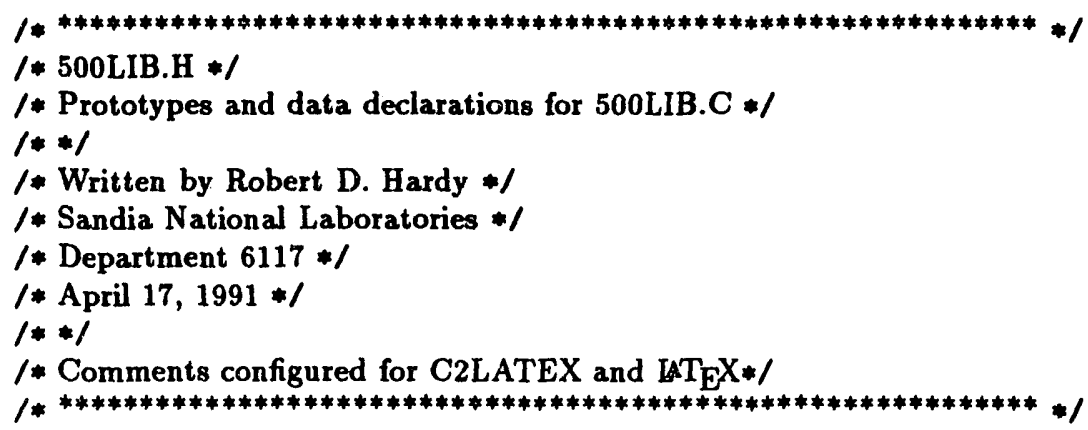

/* Timer value structure. This must be allocated by the calling program. */

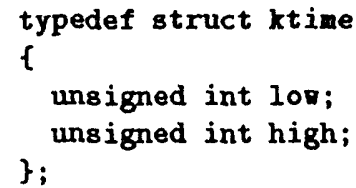

$/ *$ define pointers to interface functions. */ 


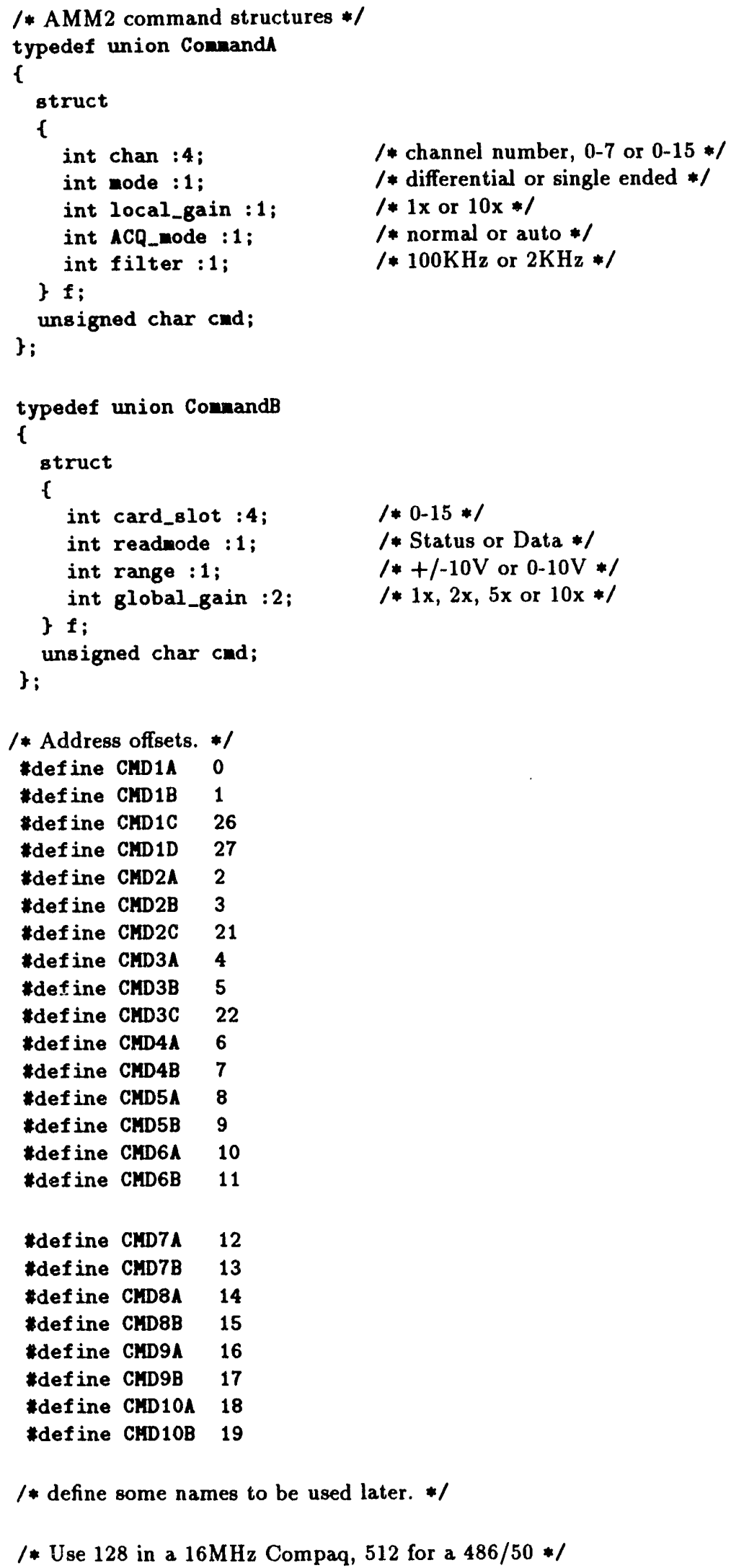


*def ine SIXTEEH

$512 / *$ delay count for $16 \mathrm{uS}$ delay in read_ad ()$* /$

tefine EOC_mask

$0 \times 80 / *$ mask to isolate EOC bit */

/* Global gain command codes. */

$\begin{array}{lll}* \text { def ine } \times 1 & 0 & / * \text { Set } 1 \times \text { gain } * / \\ \text { *def ine } \times 2 & 1 & / * \text { Set } 2 X \text { gain } * / \\ \text { *def ine } \times 5 & 2 & / * \text { Set } 5 \times \text { gain } * / \\ \text { *def ine } \times 10 & 3 & / * \text { Set } 10 X \text { gain } * /\end{array}$

/* array of board specific command addresses */

EXTERI unsigned char far *ports[11] [4];

/* Fixed addresses on the AMM2 */

EXTERI unsigned char far *SLOT;

EXTERH unsigned char far *AD_LOW;

EXTERN unsigned char far $* A D_{-} H I G H$;

EXTERH unsigned char far *AD_RECAL;

EXTERI unsigned char far *AD_START;

EXTERU unsigned char far *AD_STAT;

EXTERH unsigned char far *DA_STROBE;

EXTERH unsigned char far *EOC;

/* Fixed addresses on the interface board */

EXTERH unsigned char far *COUNTERO;

EXTERU unsigned char far *COUNTER1;

EXTERH unsigned char far *COURTER2;

EXTERN unsigned char far *COUNT_CONT;

EXTERX unsigned char far *TIMER_GLOBAL;

EXTERN unsigned char far *TIMER_STAT;

EXTERy unsigned char far *SET_IXT;

tundef EXTERH

*endif $/ *$ K500LIB *I 


\section{500LIB.C}

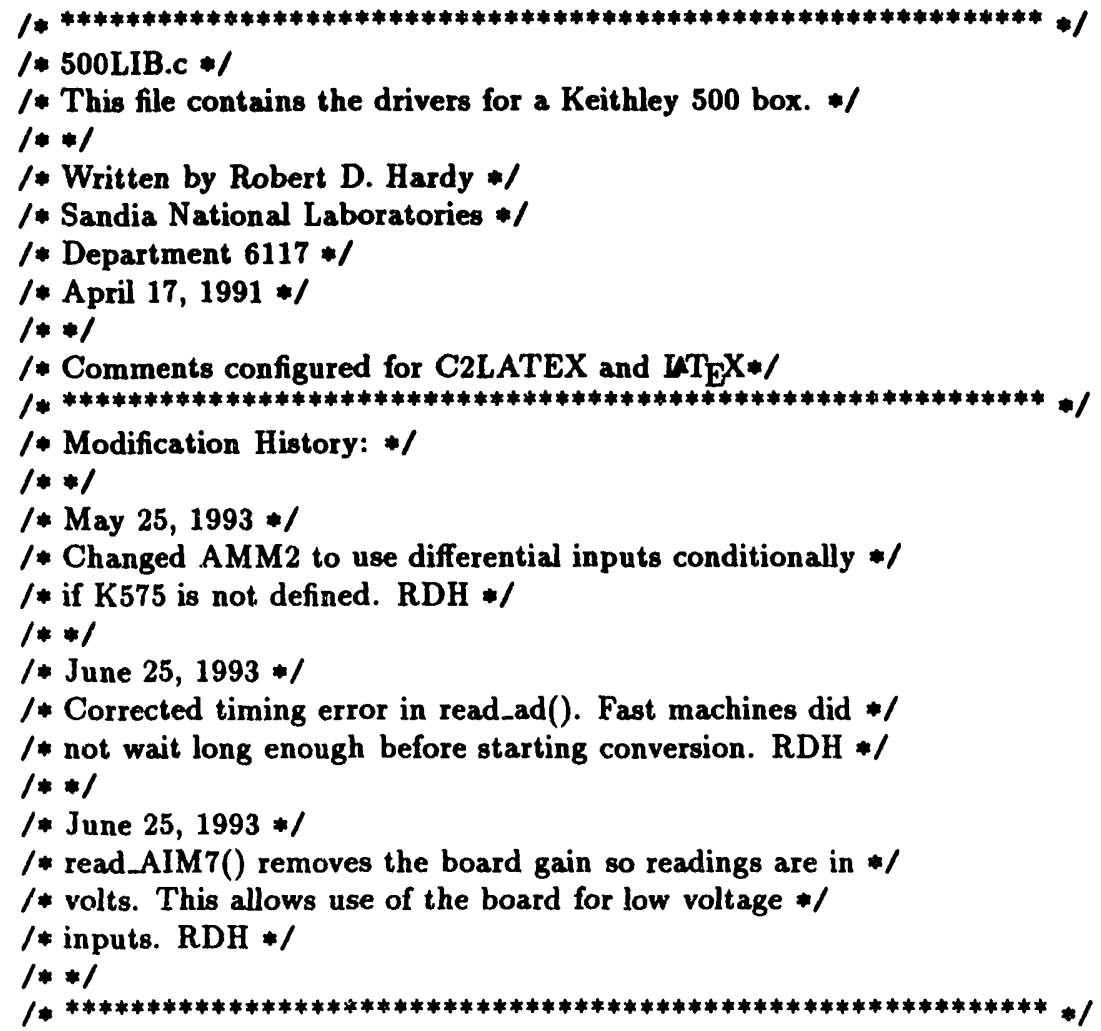

$\begin{array}{ll}\text { *define MAIM } & / * \text { provide storage for device pointers } * / \\ \text { \#define K500LIB } & / * \text { provide definitions for our data } * / \\ \text { *include "500lib.h" } & \end{array}$

/* Keithley interface segment */ static unsigned int K500_SEG = 0xcff8;

/* AMM2 command buffers */ static union Conmand $A$; static union ComandB B;

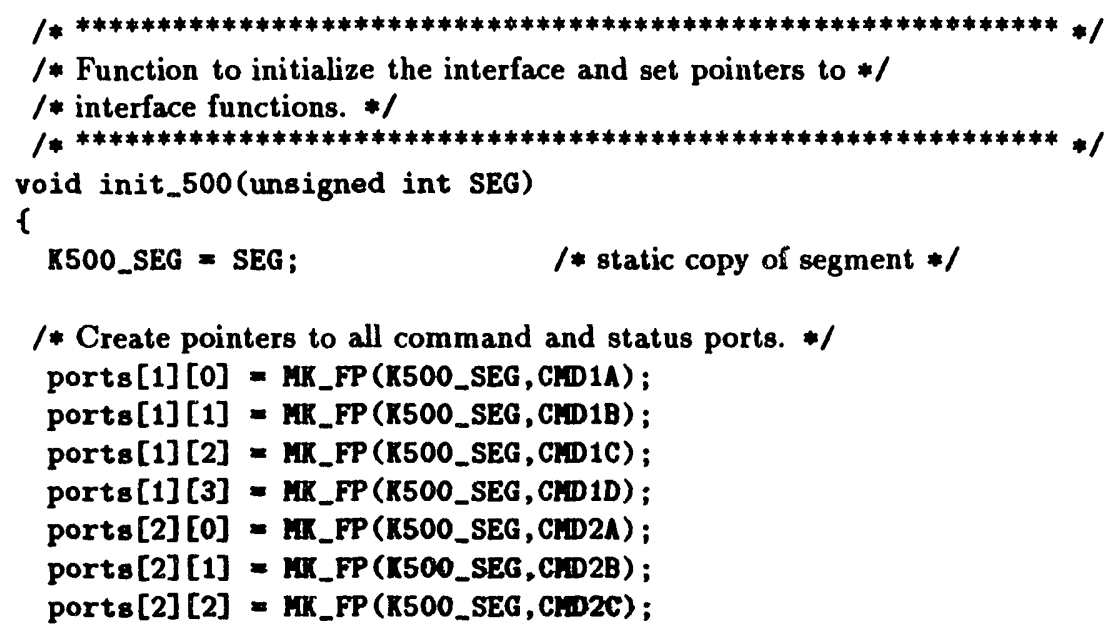




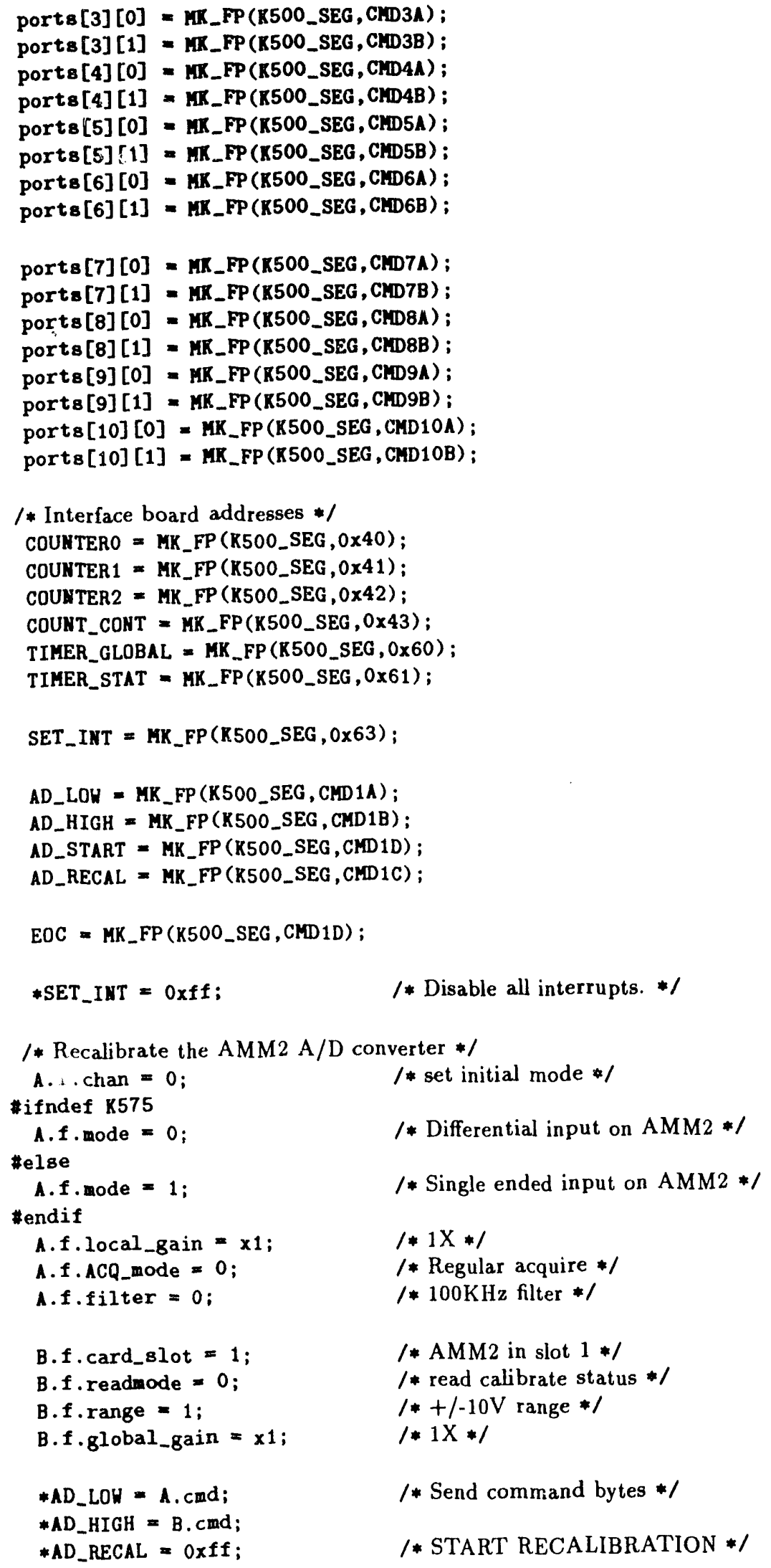




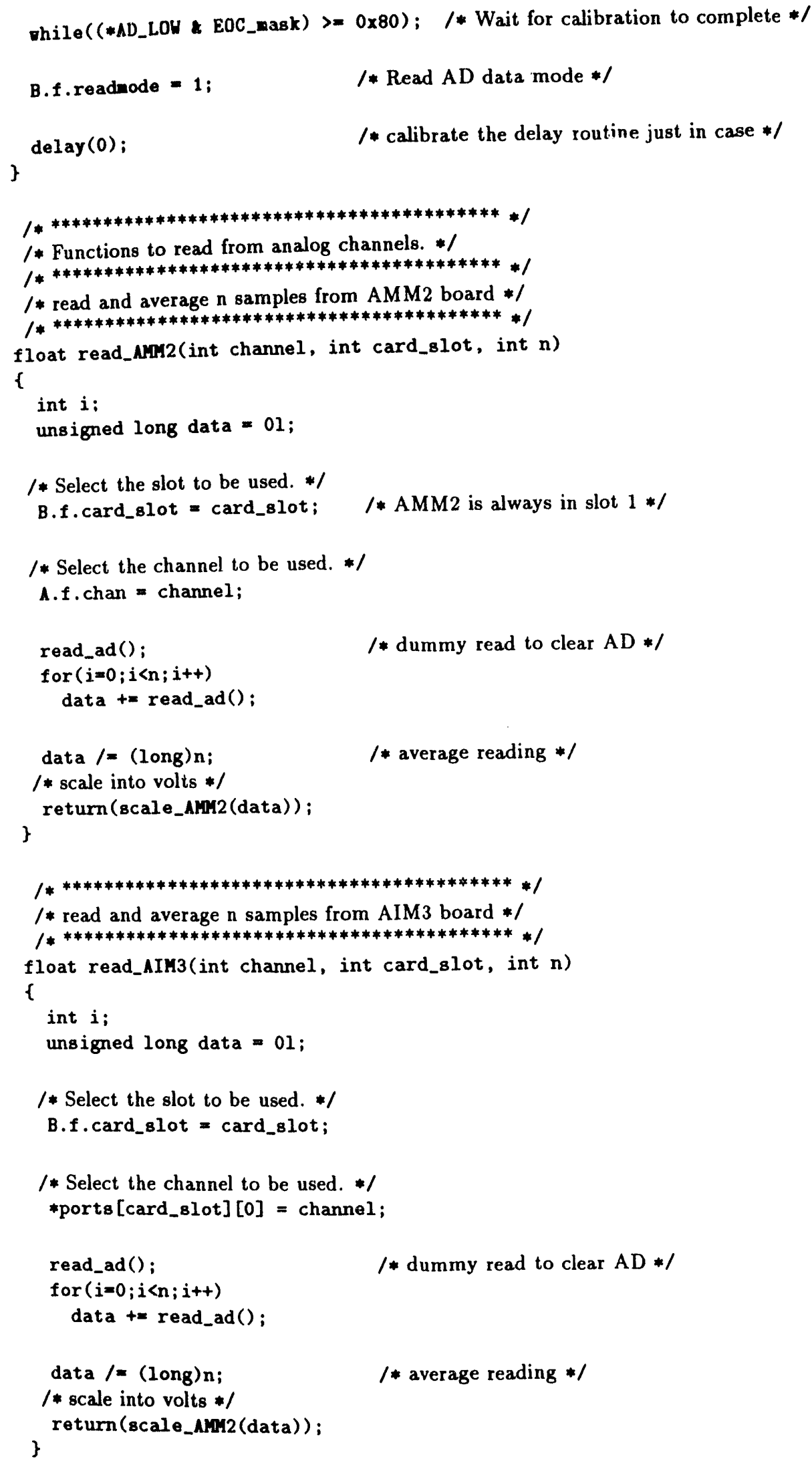




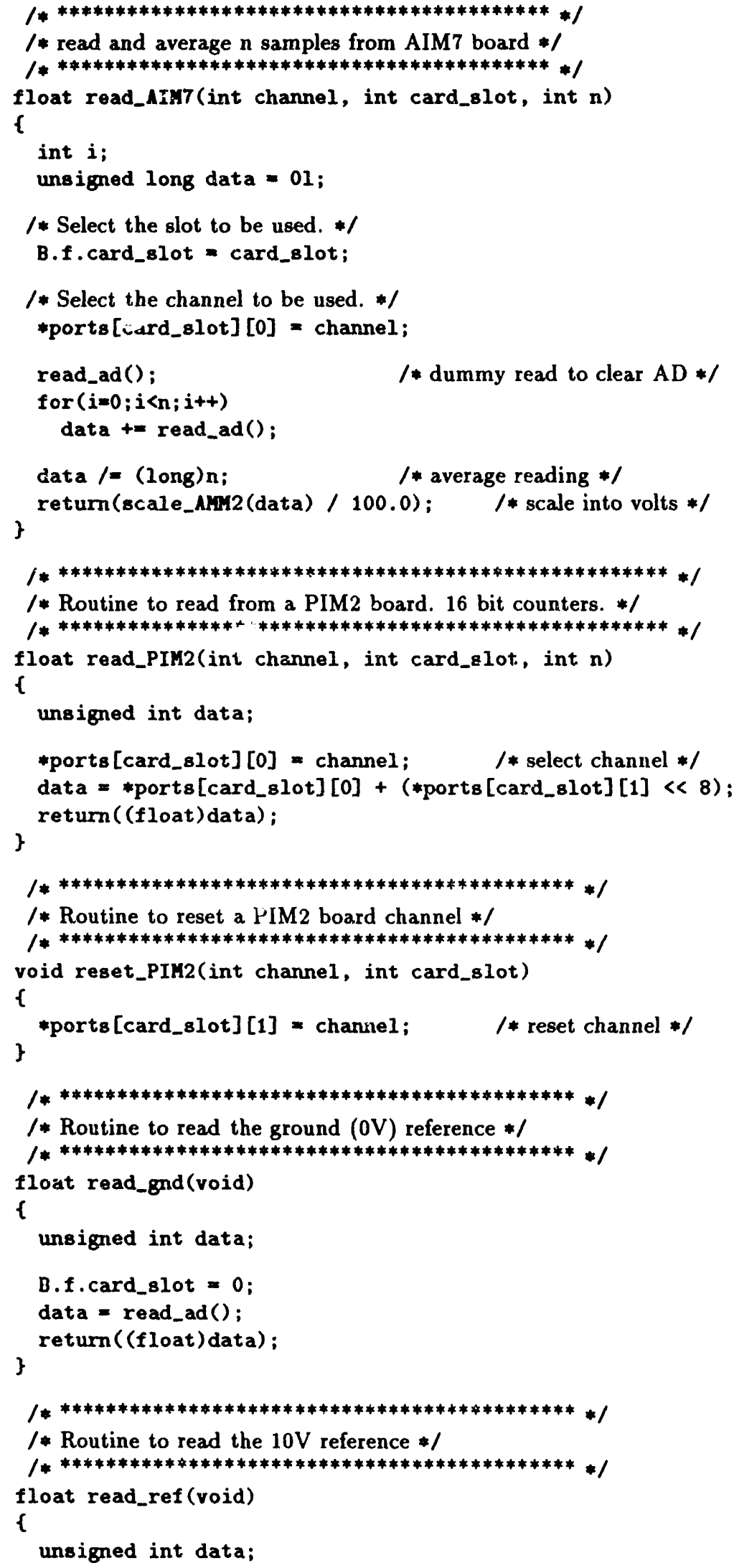




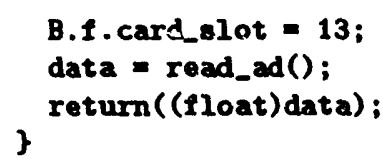




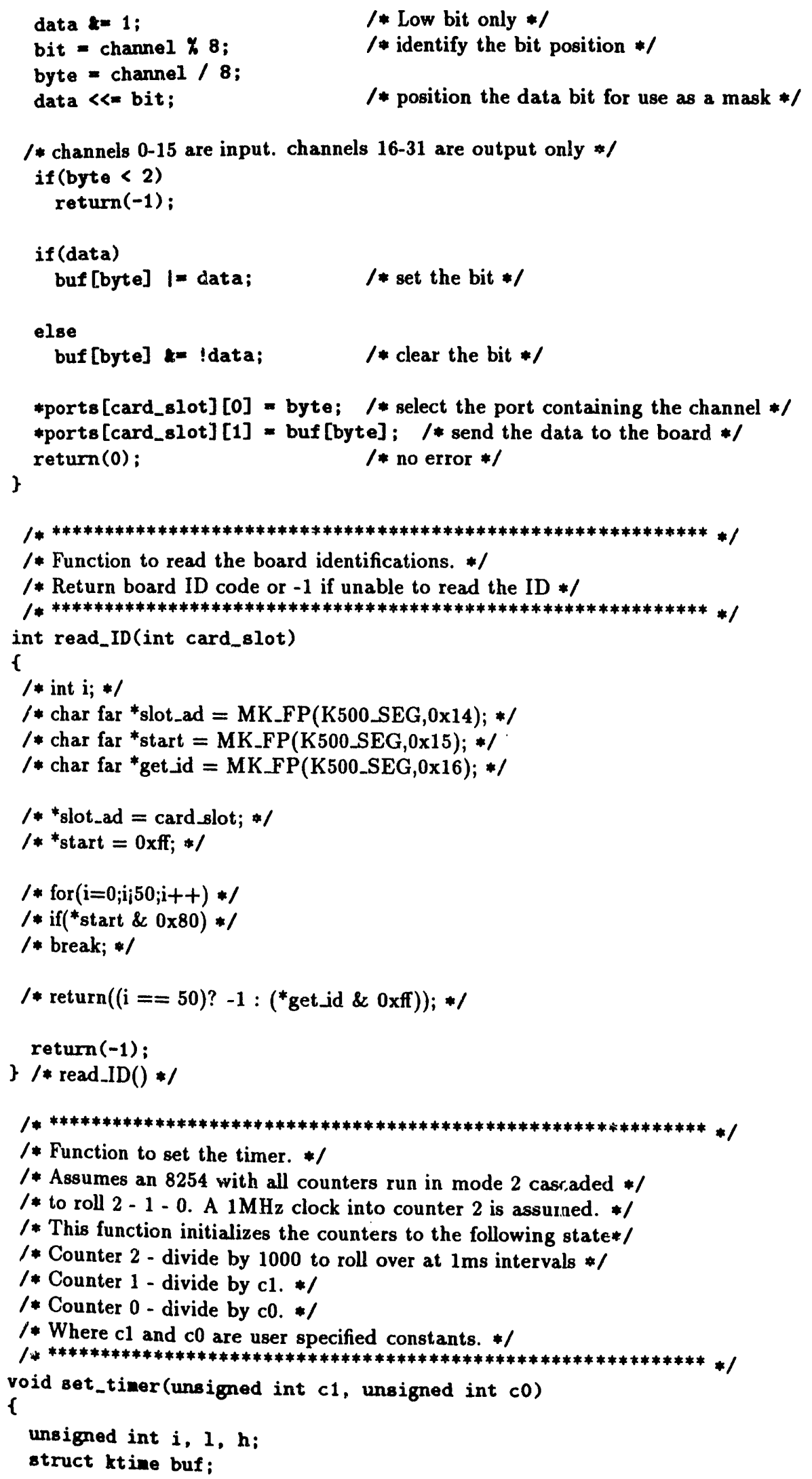




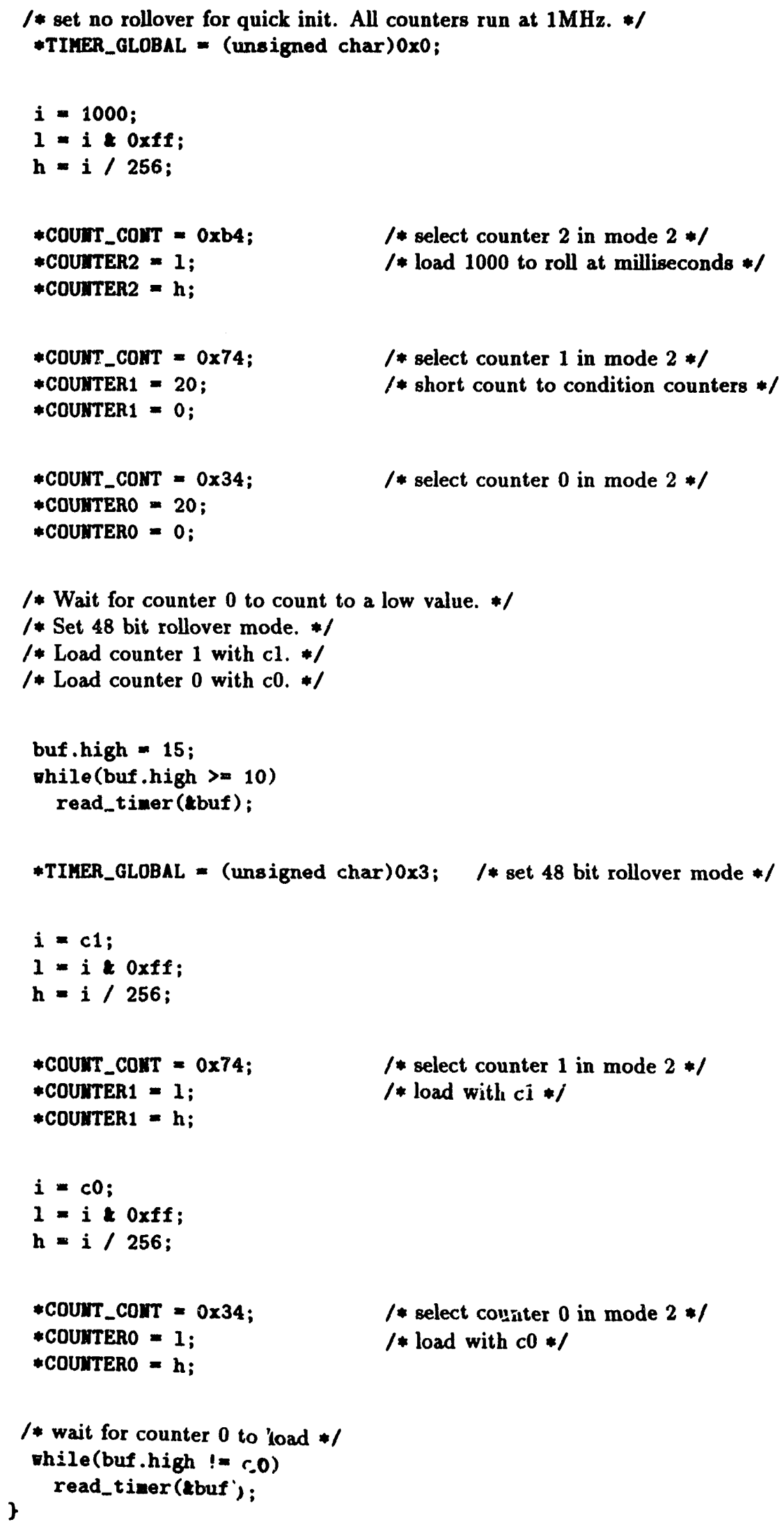




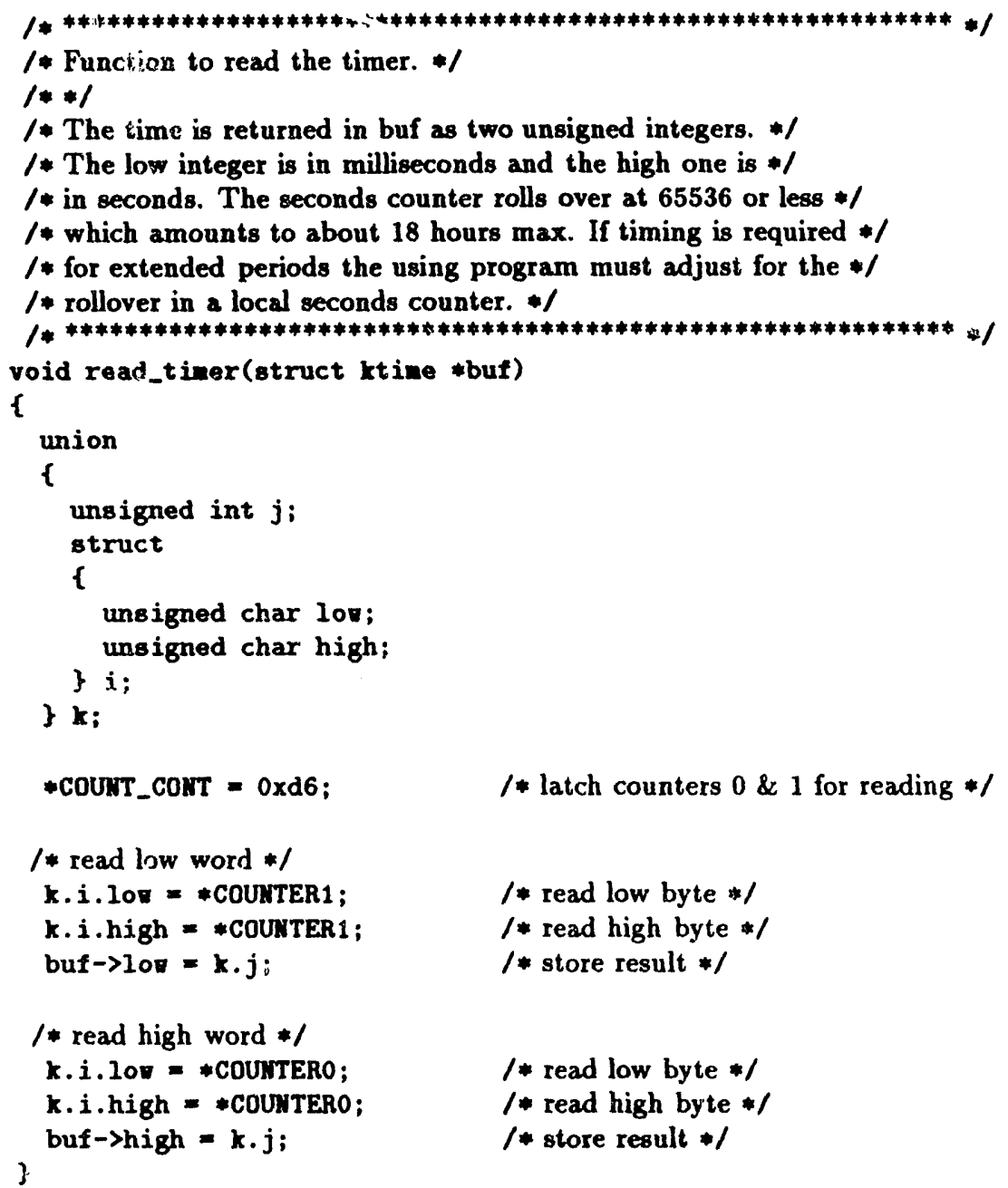




\section{E SRBGRAPH.H}

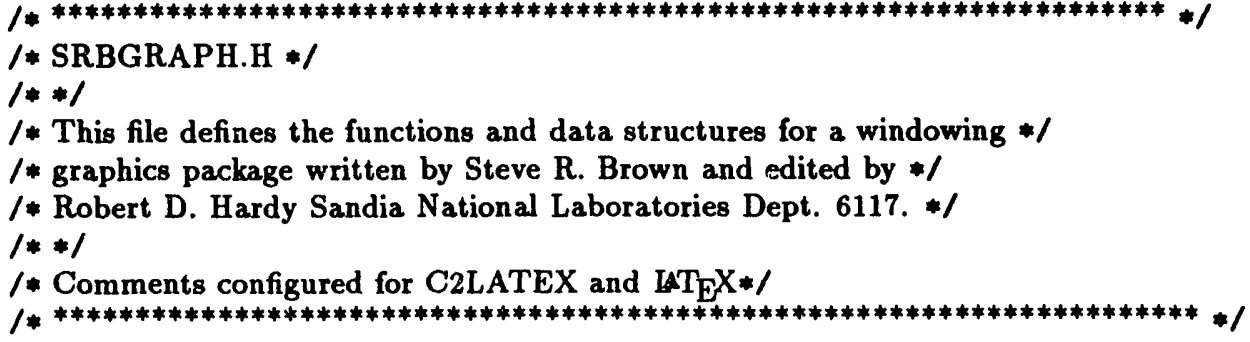


XCURSOR, YCURSOR,

MAXWIMDOH, INDEX,

FIRSTCURSOR,

BACKGROUIDCOLOR,

UINDOUCOLOR,

AXISCOLOR,

DRAWCOLOR,

Our_Font, Hn, Hd, Vn, Vd, Tw, Th; /*text scaling and font $* /$

EXTERH void *CURSOR;

void srbgraph_make_near_string (char *header, char far *hdr, int $n$ );

*endif /* GRAPH_LIB */

/* FUNCTION PROTOTYPES */

void far data(void);

void far error (char far *string);

void far open_graph(void);

void far dray_border(int SXmin, int SYmin, int SXmax, int SYmax, char far theader);

void far define_vindor(int vindov, int SXmin, int SYmin,

int sXmax, int SYmax,

float UXmin, float UYmin,

float UXmax, float UYmax);

void far select_vindor(int vindor);

void far define_header (int vindov, char far $*$ hdr);

void far coord_display (float xc, float yc, char far *c);

void far erase_coord_display(void);

void far reset_windors (void);

void far erase_vindov(int vindov);

int far windor $x$ (float $x)$;

int far vindov_y(float $y)$;

void far draw_axes (int vindor, int ticksx, int ticksy,

char $f$ ar *namex, char far *namey);

void far drav_point (float $x r$, float $y r)$;

void far dras_line(float UXmin, float UYmin, float UXmax, float UYmax);

void far dran_line_to(float xr, float yr);

void far move_to(float $x r$, float $y r)$;

void far dras_square(float $x r$, float yr);

void far dran_x(float $x r$, float $y r$ );

void far dras_triangle(float $x r$, float $y r$ );

void far drav_dianond(float $x r$, float yr);

void far dran_circle(flcat xr, float yr);

void far drav_cross (float xr, float yr);

void far dran_star(float xr, float yr);

void $f$ ar nake_cursor(void);

void far put_cursor(float $x$, float $y$ );

void far erase_cursor (void);

void far free_cursor(void);

void far menu_bar(int top, int $n$, char far *text []); 


\section{F SRBGRAPH Composite Source}

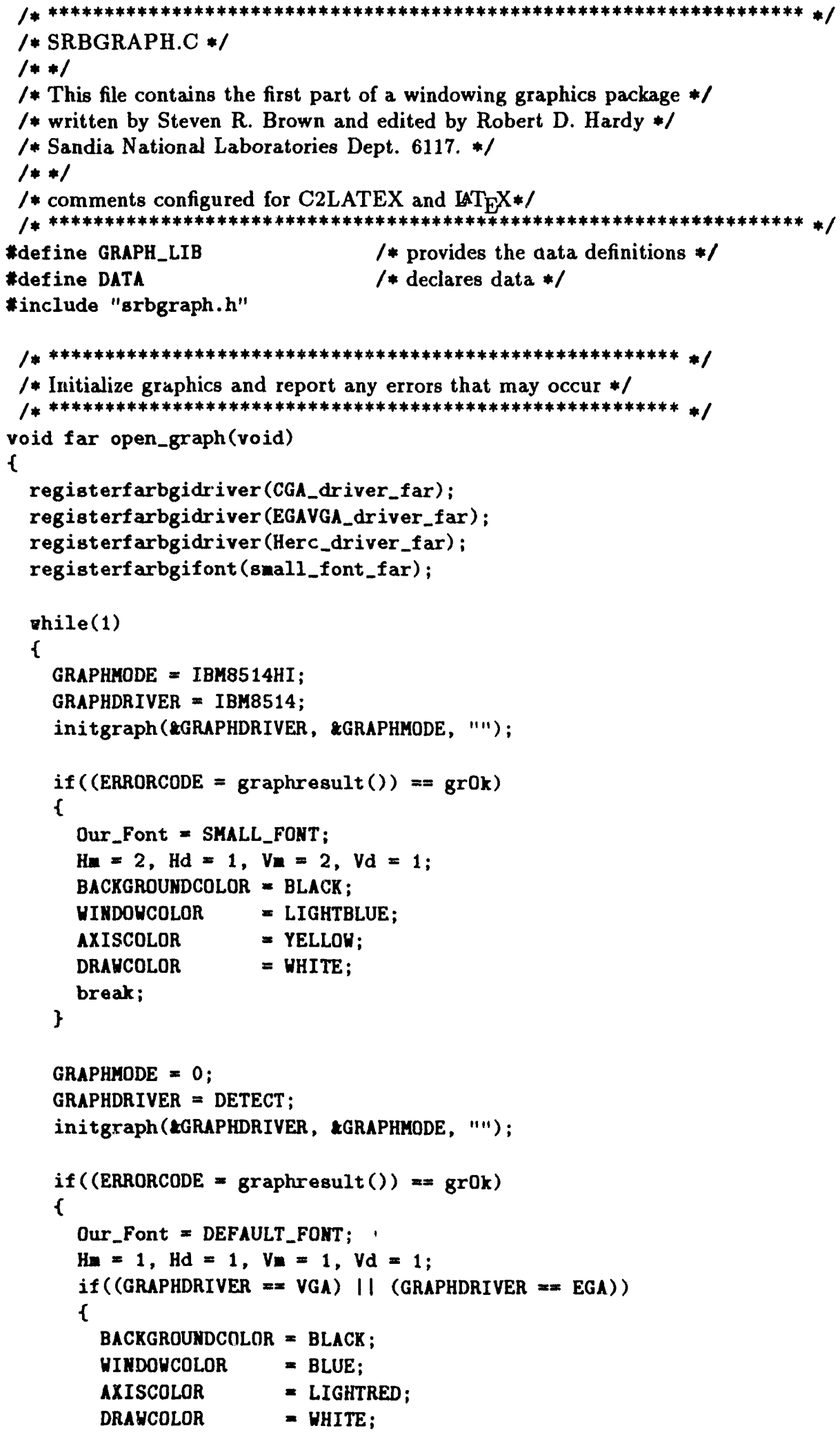




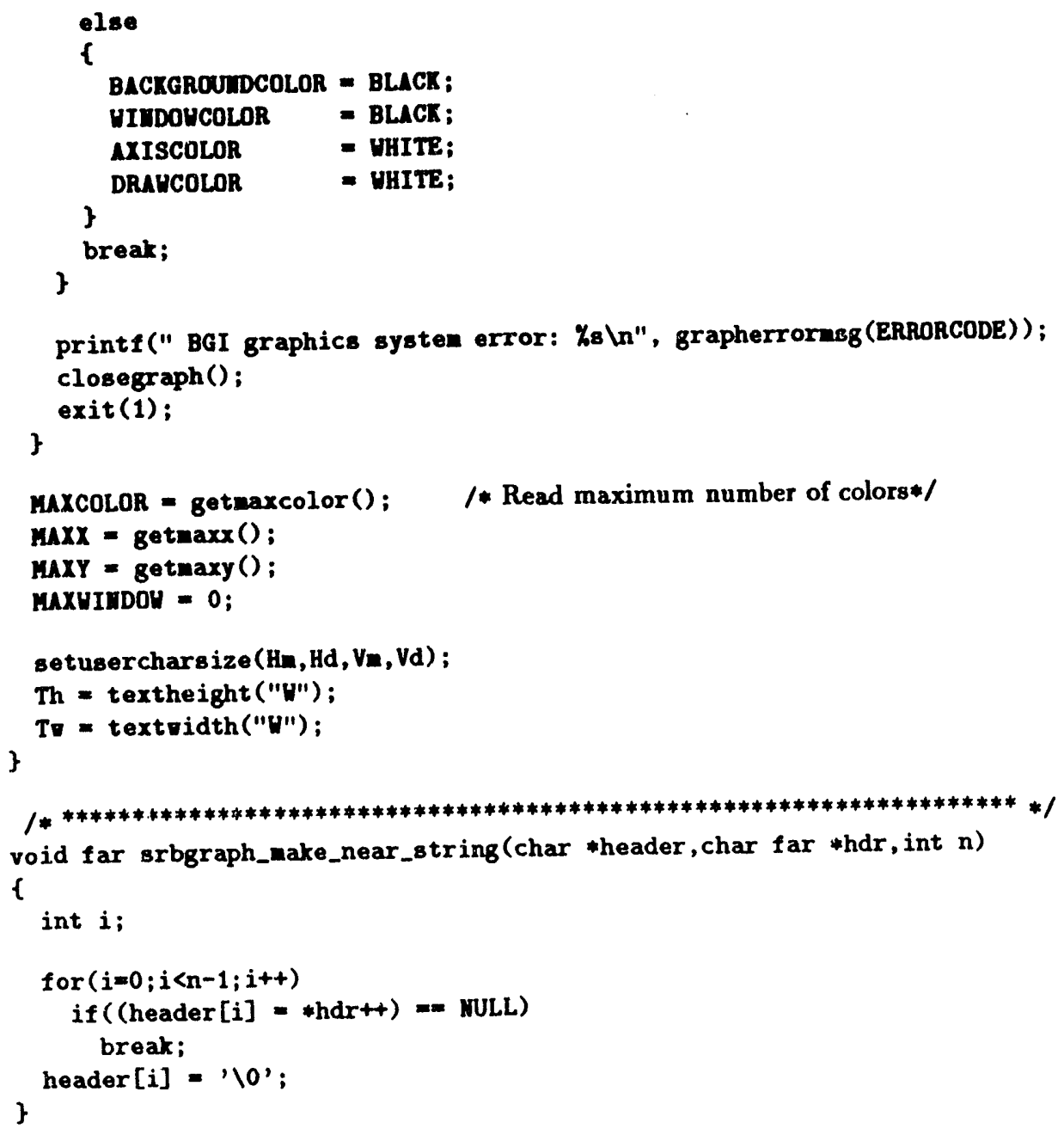

\section{F .1 DEFINE.C}

$/ * * * * * * * * * * * * * * * * * * * * * * * * * * * * * * * * * * * * * * * * * * * * * * * * * * * * * * * * * * * * * * * * * * * /$

/* DEFINE.C */

$1 * * 1$

/* This file contains part of a windowing graphics package written by $* /$

/* Steven R. Brown and edited by Robert D. Hardy */

/* Sandia National Laboratories Dept 6117. */

$1 * * 1$

/* comments configured for C2LATEX and LTTE* $\mathrm{X} * 1$

$/ * * * * * * * * * * * * * * * * * * * * * * * * * * * * * * * * * * * * * * * * * * * * * * * * * * * * * * * * * * * * * * * * * * * * * 1$

tdefine GRAPH_LIB

$1 *$ pzovides the data definitions $*$ I

\#include "srbgraph.h"

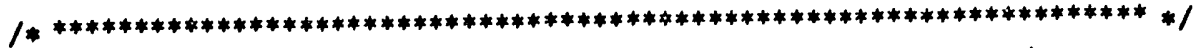

$/ *$ fill in the WINDINFO structure for this window if all parameters */

$1 *$ are valid $* 1$

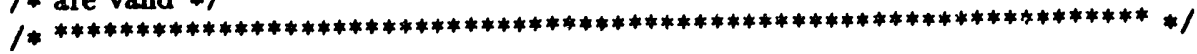

void far define_vindor(int windor,

int sXmin, int sYain, int sxmax, int SYmax,

flcat UXmin, float UYmin, float UXmax, float UYmax)

\{ 


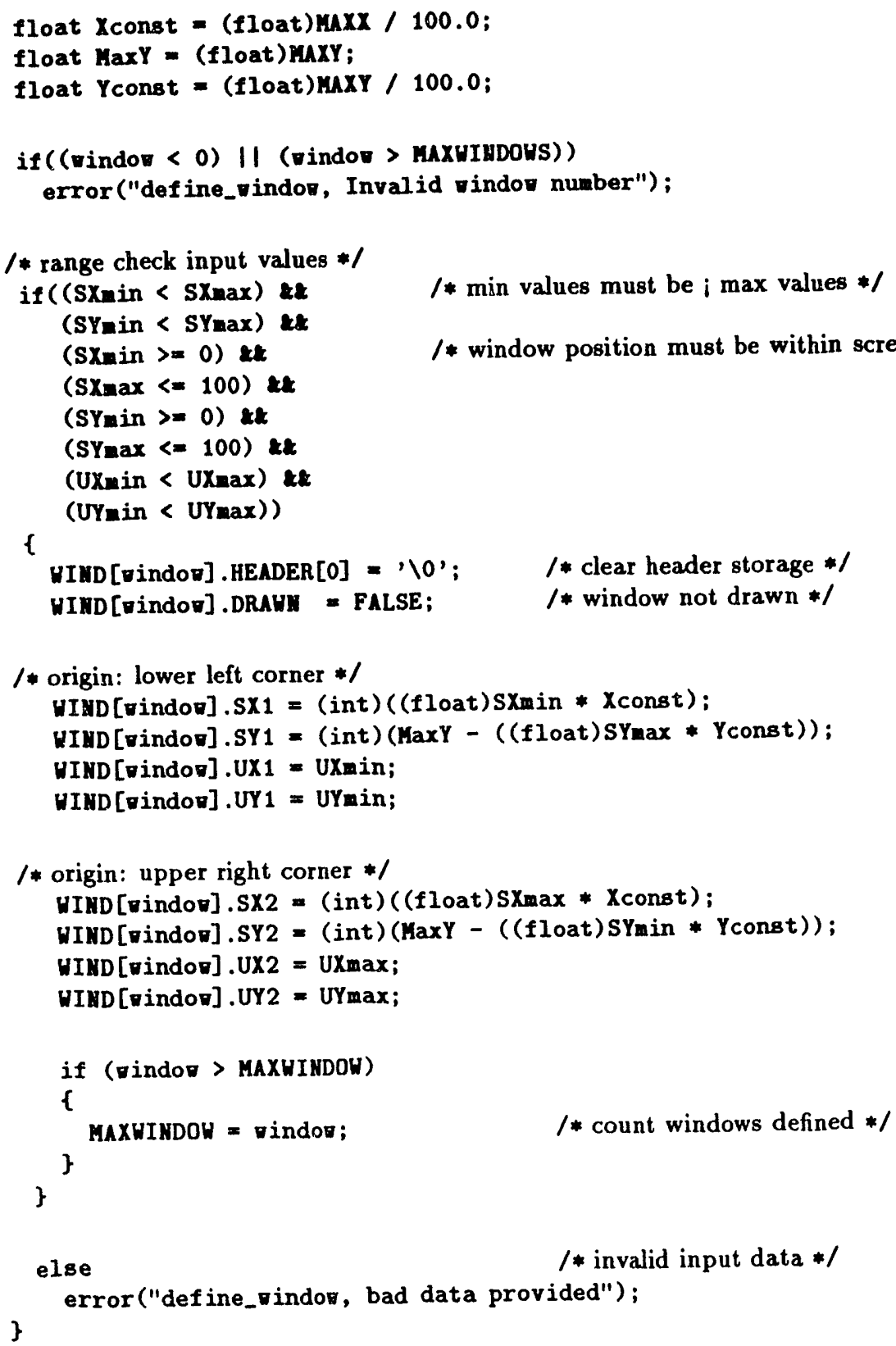

\section{F .2 DEF_DHR.C}

/*********************************************************************/

/*DEF_HDR.C*/

$1 * * 1$

/* This file contains part of a windowing graphics package written by $* /$

/* Steven R. Brown and edited by Robert D. Hardy */

/* Sandia National Laboratories Dept. 6117. */

$1 * * 1$

/* comments configured for C2LATEX and IAT $\mathrm{EX} * 1$

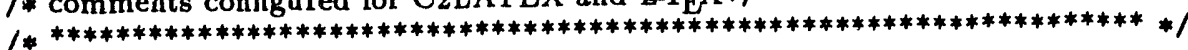

\#define GRAPH_LIB

$/ *$ provides the data definitions $* /$

\#include "srbgraph.h" 


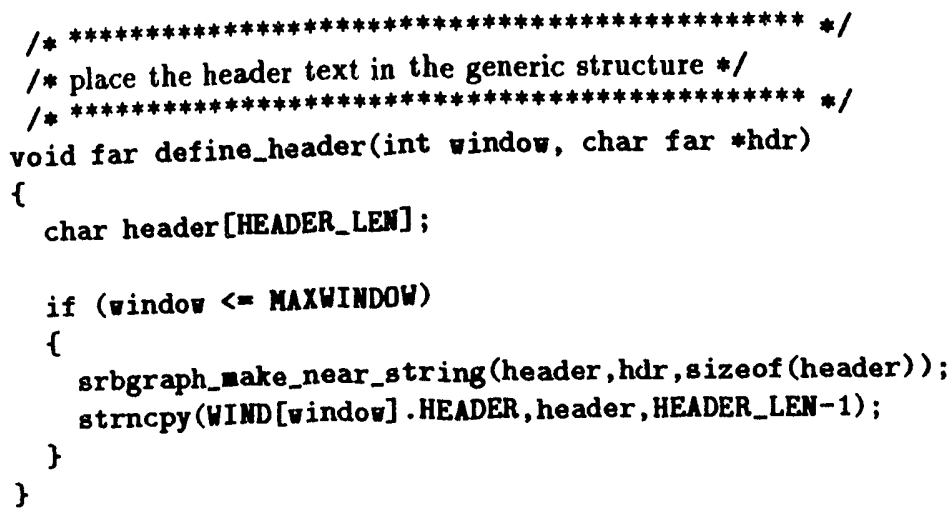

\section{F .3 DRAWAXIS.C}

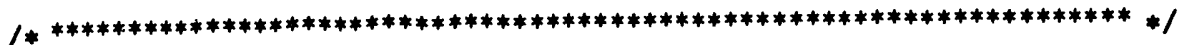

/* DRAWAXIS.C *I

$/ * * 1$

/* This file contains part of a windowing graphics package written by */

/ Steven R. Brown and edited by Robert D. Hardy */

/* Sandia National Laboratories Dept. 6117. */

$1 * * 1$

/* Comments configured for C2LATEX and IAT $_{\mathrm{E}} \mathrm{X} * 1$

$/ * * * * * * * * * * * * * * * * * * * * * * * * * * * * * * * * * * * * * * * * * * * * * * * * * * * * * * * * * * * * * * * * * * * * * 1$

*define GRAPH_LIB

/ provides the data definitions */

*include "srbgraph.h"

static void far drav_x_axis (int number_of_ticks, char far *nane);

static void far drav_y_axis (int number_of_ticks, char far *nane);

static void scale_axes (char **text, int $n$, float MIH, float MAX);

static void aake_X_labels (int number_of_ticks);

static void nake_Y_labels (int number_of_ticks);

static char s [HEADER_LEN];

static float $d x$, dy, delta;

static int ticks;

static char *Xptrs [25];

/* pointers used in axis labeling routines */

static char *Yptrs [25];

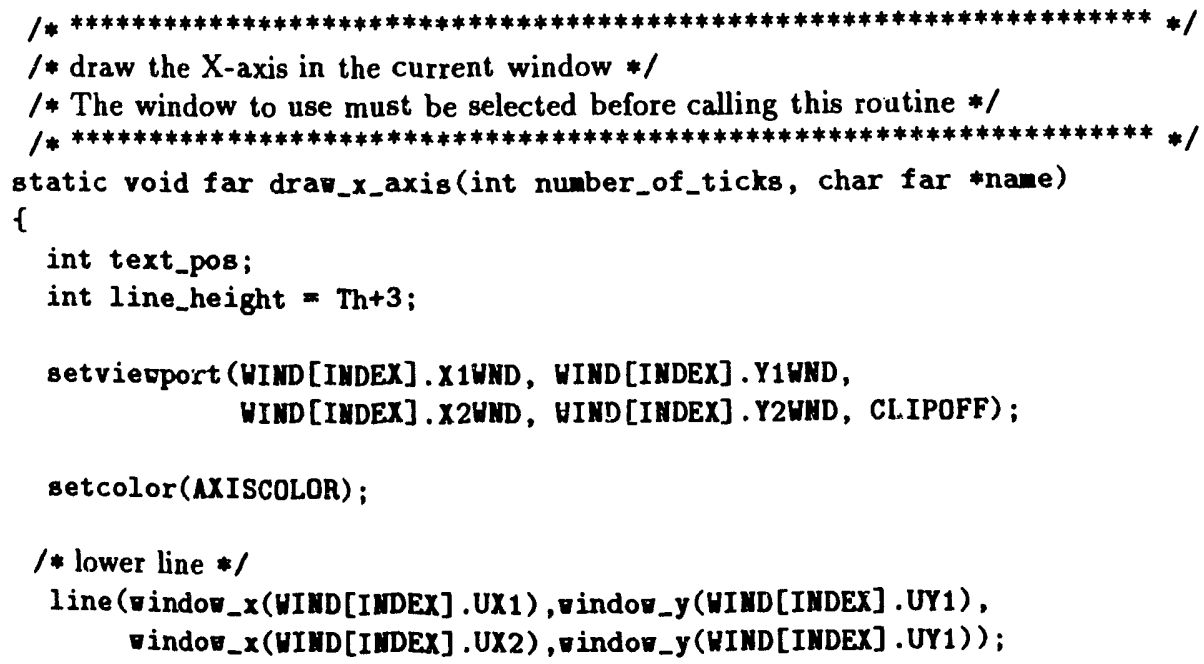




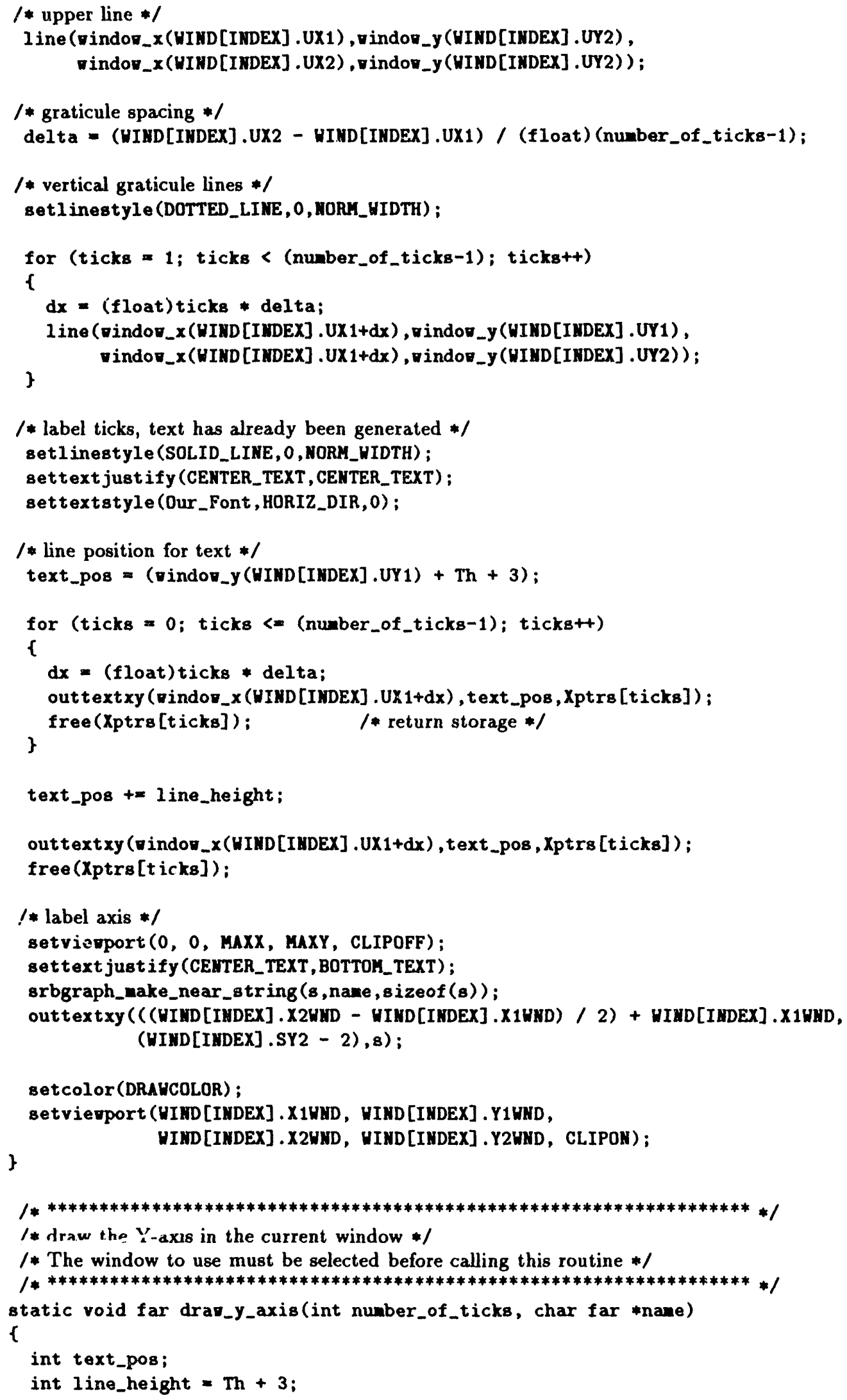




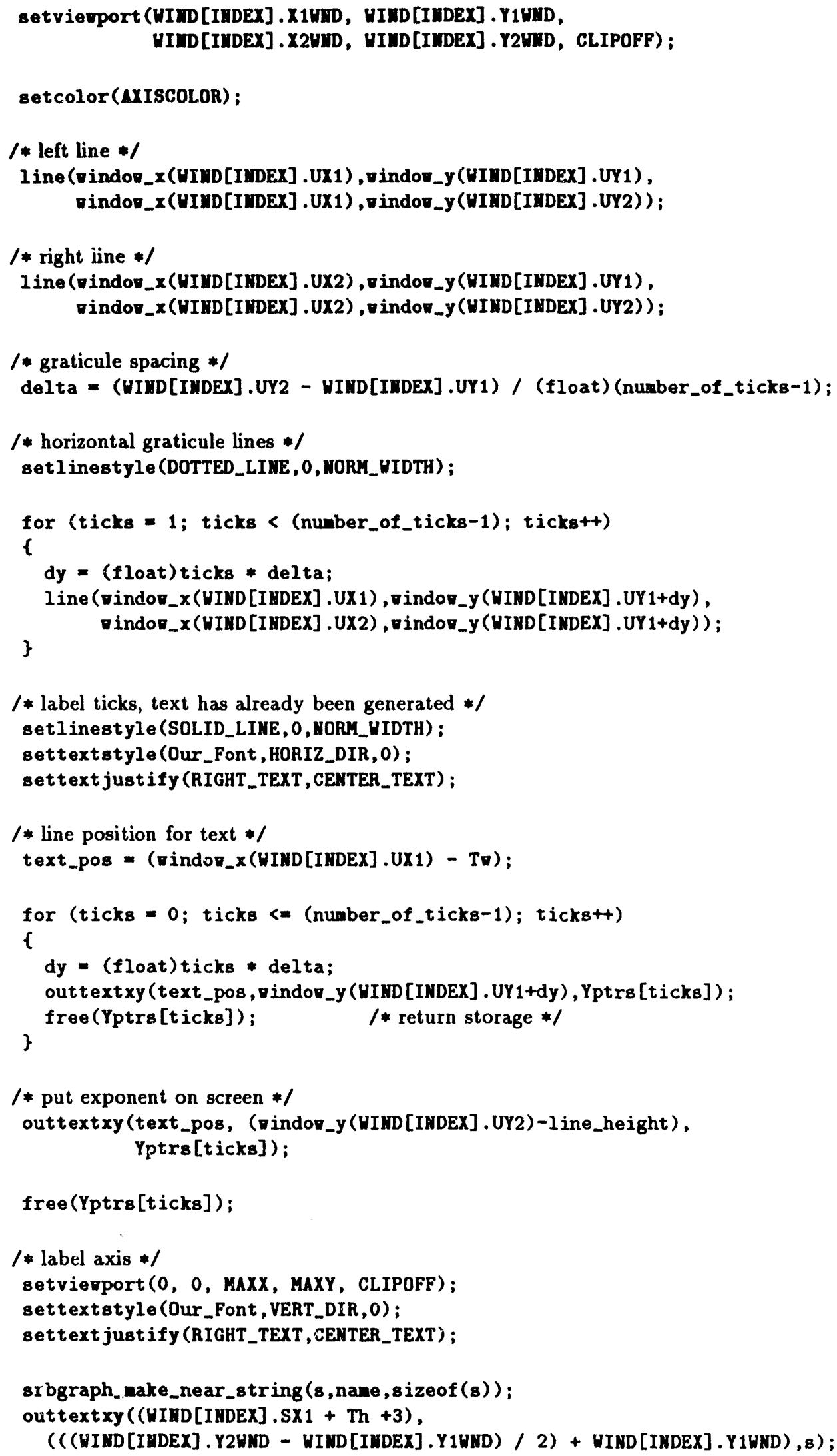




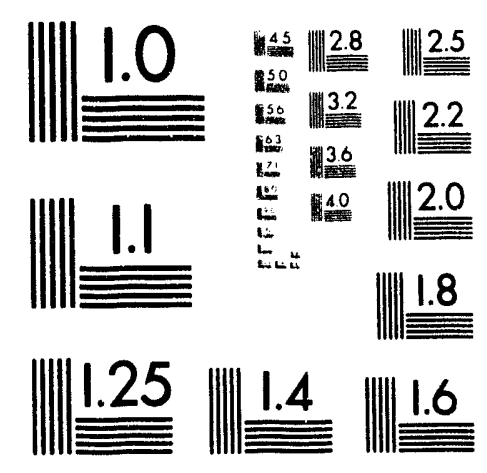



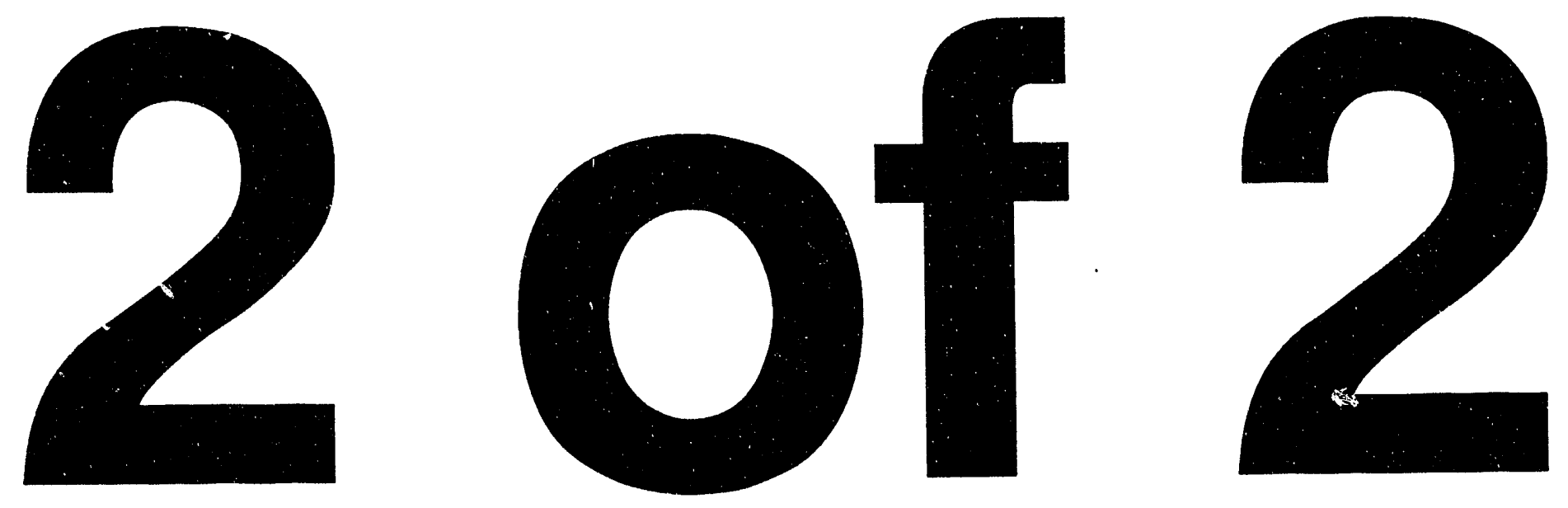


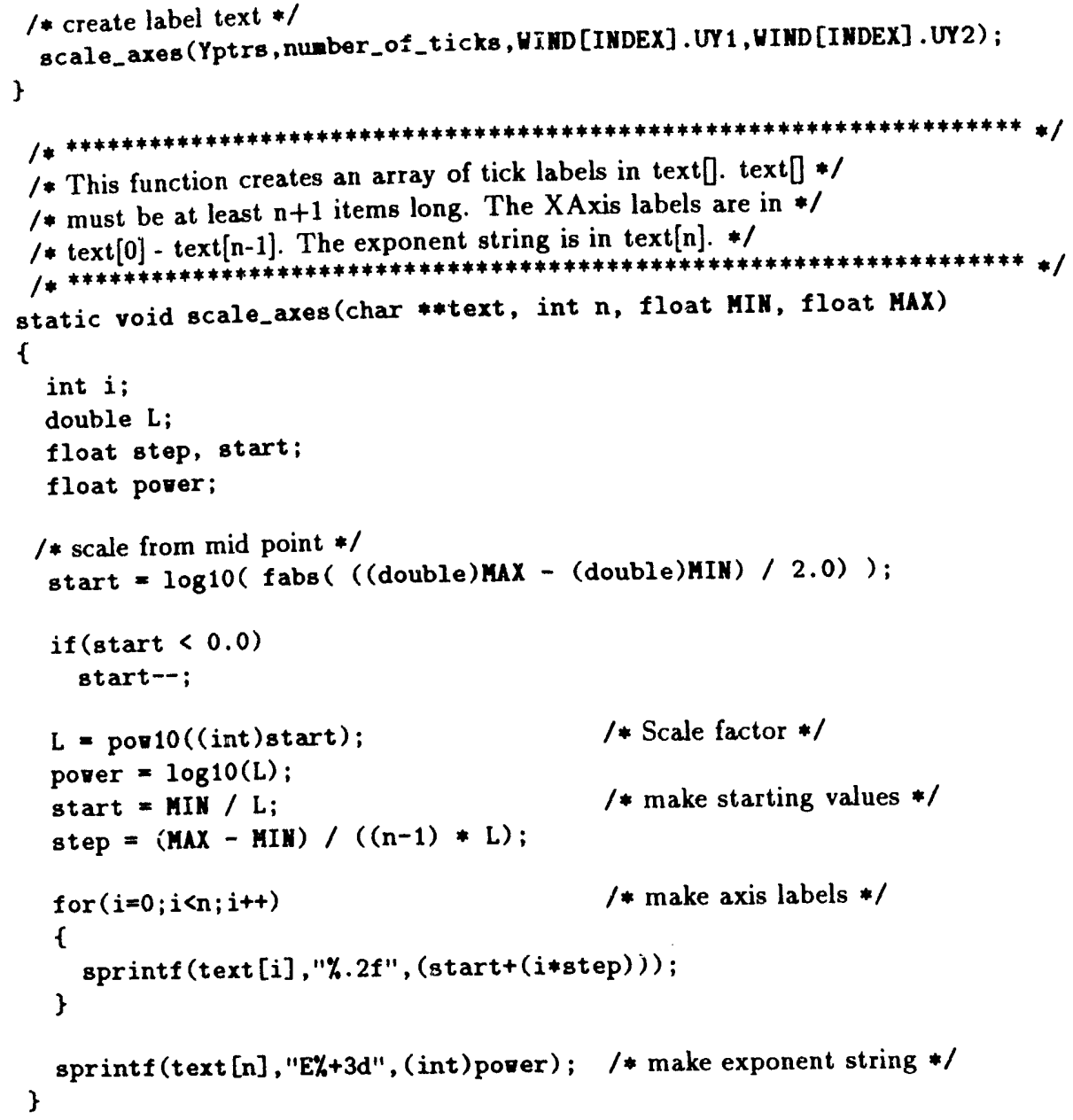

\section{F .4 DRAW_B.C}

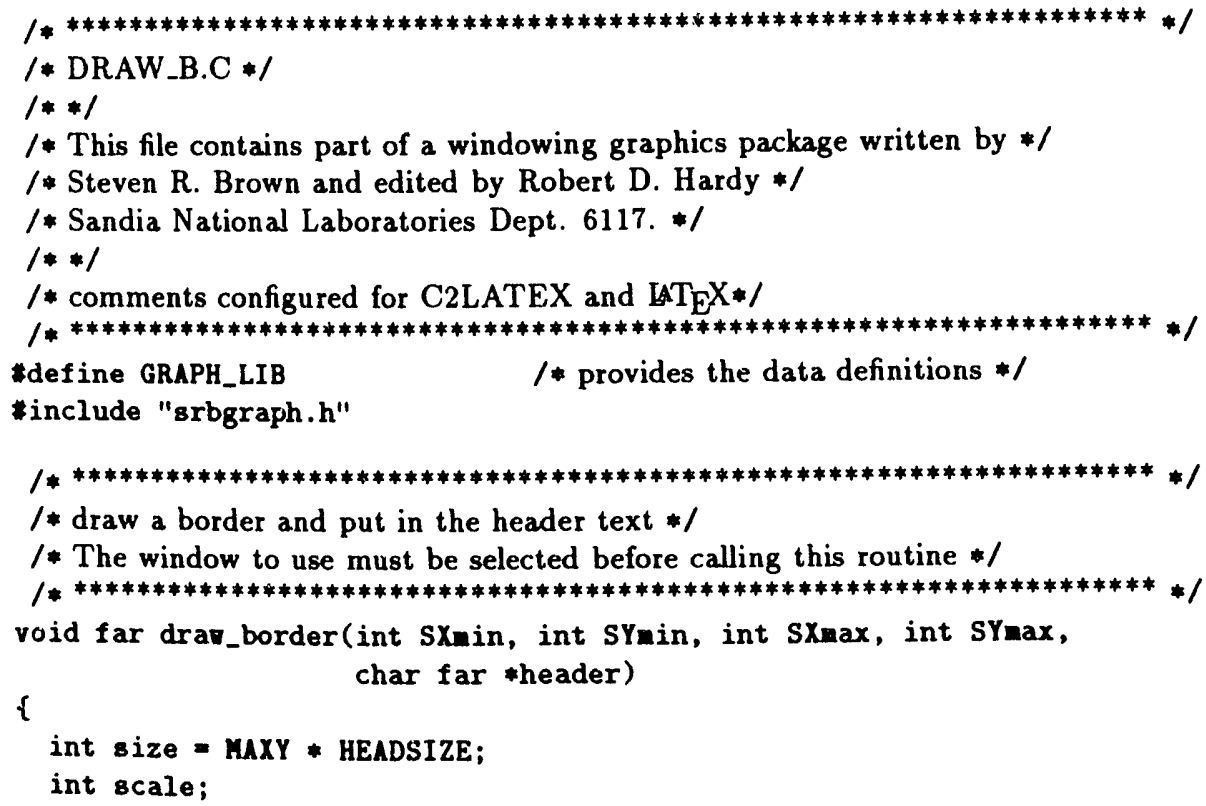




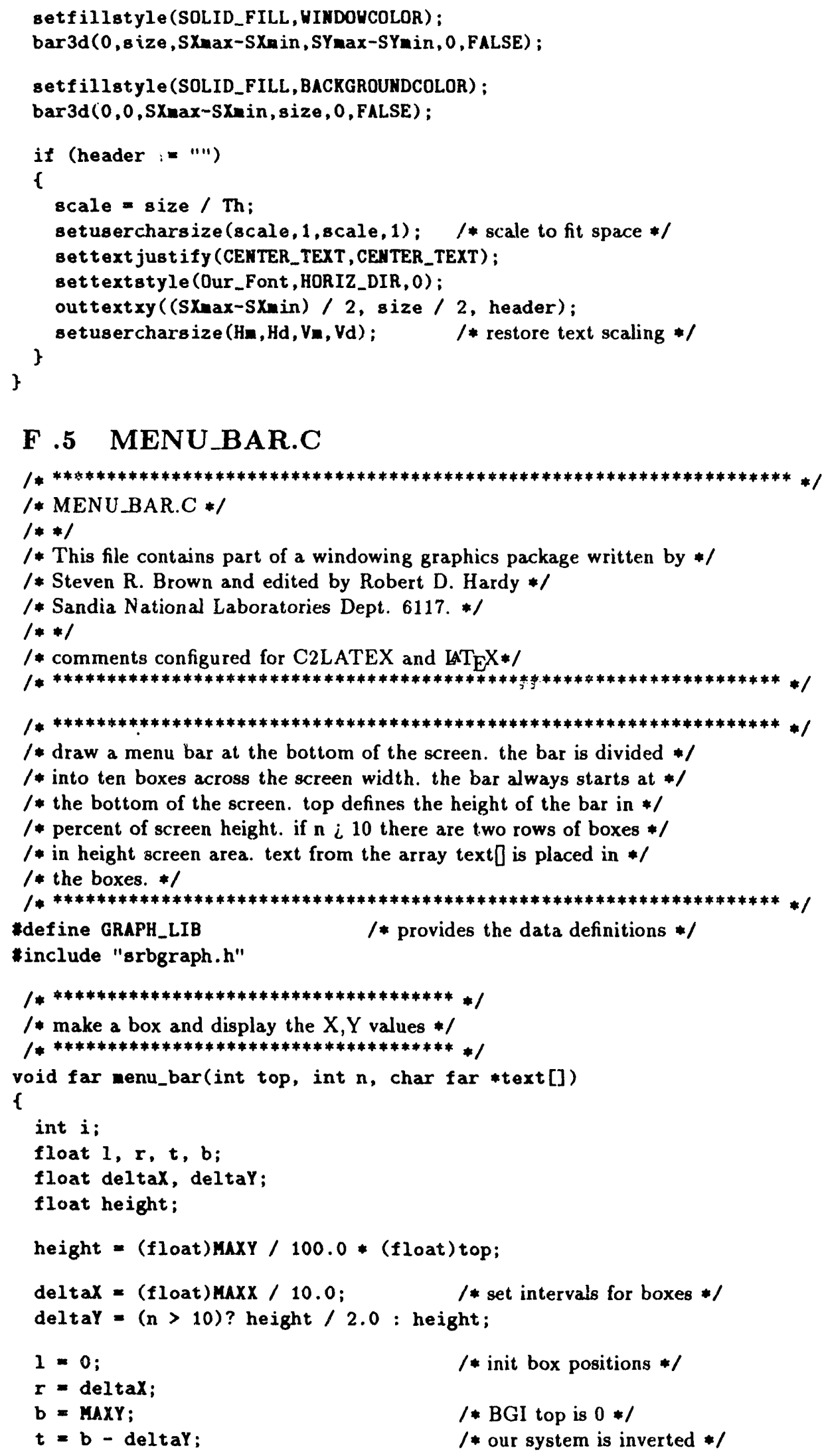

\section{F .5 MENU_BAR.C}




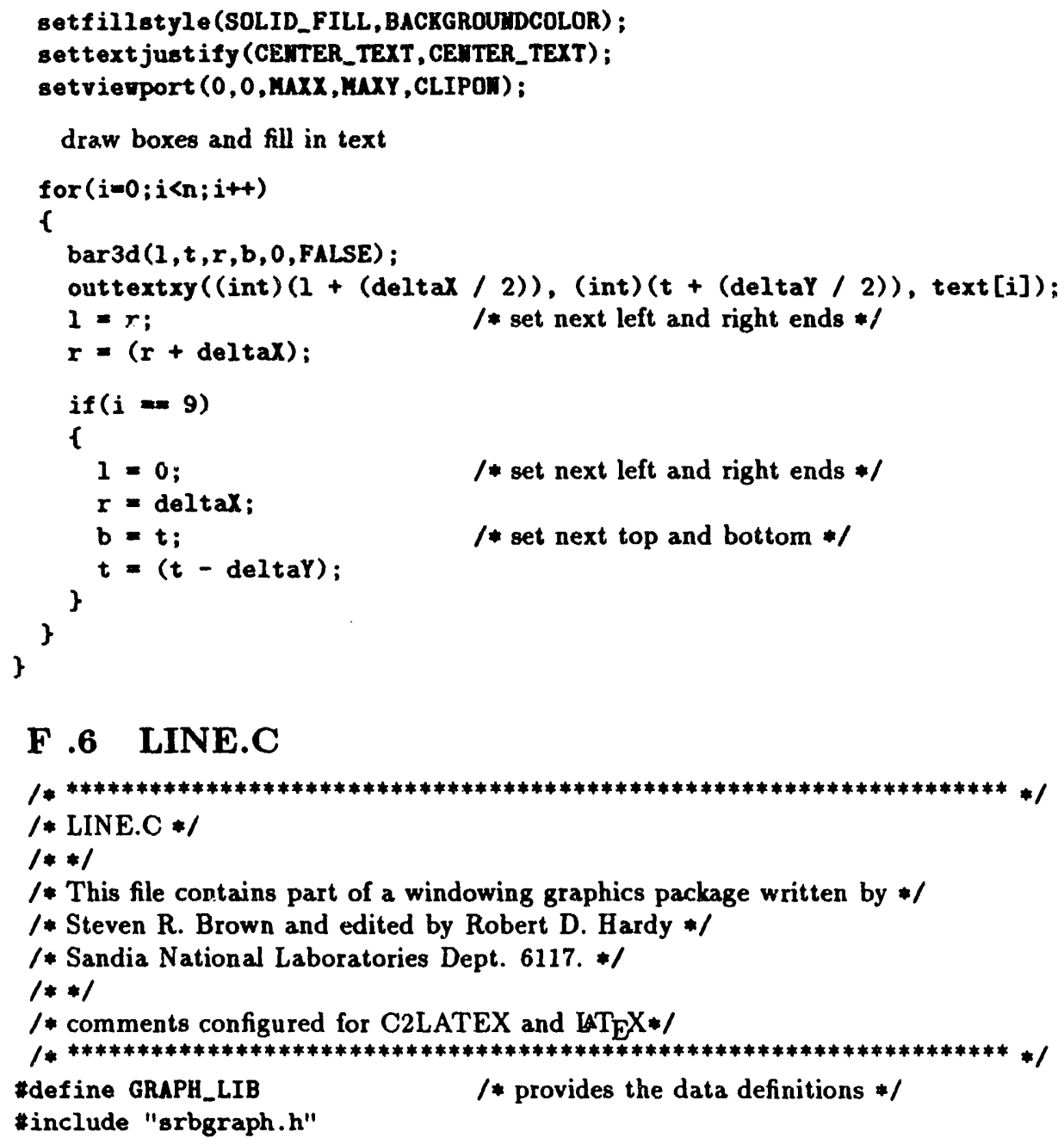

F .6 LINE.C

$/ * * * * * * * * * * * * * * * * * * * * * * * * * * * * * * * * * * * * * * * * * * * * * * * * * * * * * * * * * * * * * * * * * * * * * 1$

/* LINE.C */

$1 * * 1$

1* This file contains part of a windowing graphics package written by $*$ /

/* Steven R. Brown and edited by Robert D. Hardy */

/* Sandia National Laboratories Dept. 6117. */

$1 * * 1$

/* comments configured for C2LATEX and LAT $\mathrm{E}$ X*1

$1 * * * * * * * * * * * * * * * * * * * * * * * * * * * * * * * * * * * * * * * * * * * * * * * * * * * * * * * * * * * * * * * * * * * * * 1$

\#define GRAPH_LIB

$1 *$ provides the data definitions $* /$

\#include "srbgraph.h"

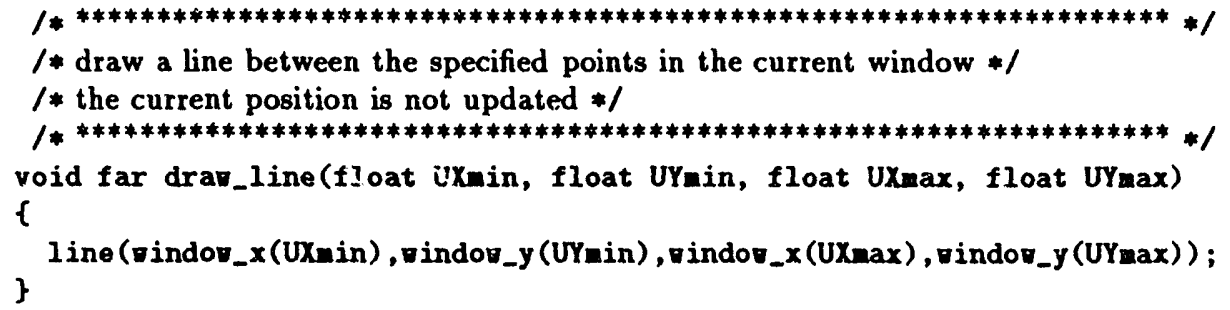

\section{F .7 LINE_TO.C}

$/ * * * * * * * * * * * * * * * * * * * * * * * * * * * * * * * * * * * * * * * * * * * * * * * * * * * * * * * * * * * * * * * * * * * * * 1$ /* LINE_TO.C */

$1 * * 1$

/* This file contains part of a windowing graphics package written by */

/* Steven R. Brown and edited by Robert D. Hardy */

/* Sandia National Laboratories Dept. 6117.*/

$1 * * 1$

/* comments configured for C2LATEX and LT $\mathrm{EX} *$ /

$1 * * * * * * * * * * * * * * * * * * * * * * * * * * * * * * * * * * * * * * * * * * * * * * * * * * * * * * * * * * * * * * * * * * * * 1$

*òefine GRAPH_LIB

\#include "srbgraph.h" 


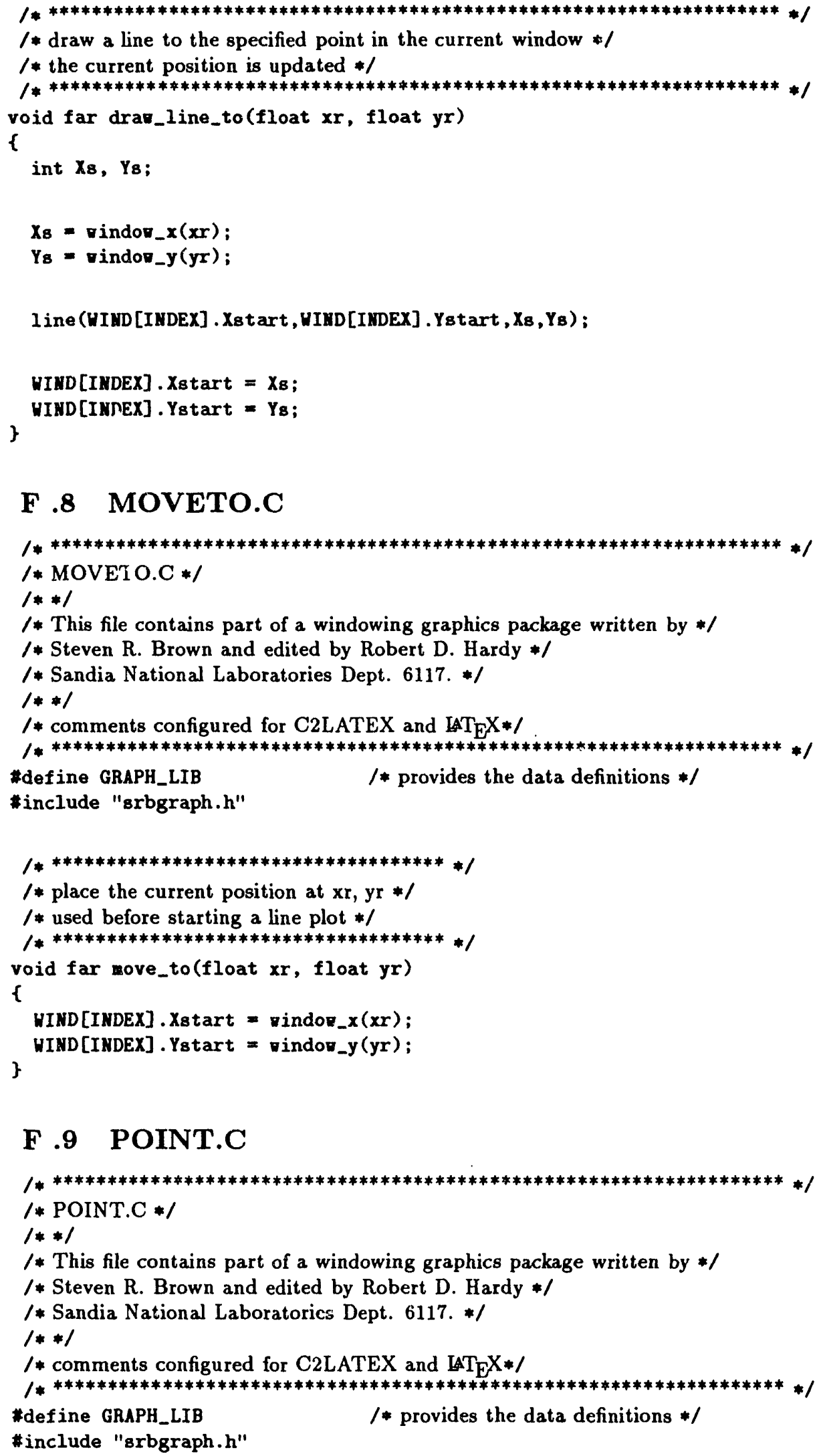

F .8 MOVETO.C

F .9 POINT.C

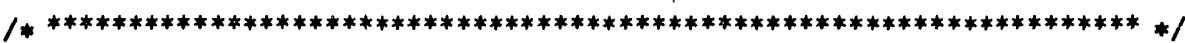
/* POINT.C *I

$1 * * 1$

/* This file contains part of a windowing graphics package written by */

/* Steven R. Brown and edited by Robert D. Hardy */

/* Sandia National Laboratorics Dept. 6117. */

$1 * * 1$

/* comments configured for C2LATEX and LAT $\mathrm{TX} * /$

$1 * * * * * * * * * * * * * * * * * * * * * * * * * * * * * * * * * * * * * * * * * * * * * * * * * * * * * * * * * * * * * * * * * * * * 1$

*define GRAPH_LIB

/* provides the data definitions $* /$

\#include "srbgraph.h" 


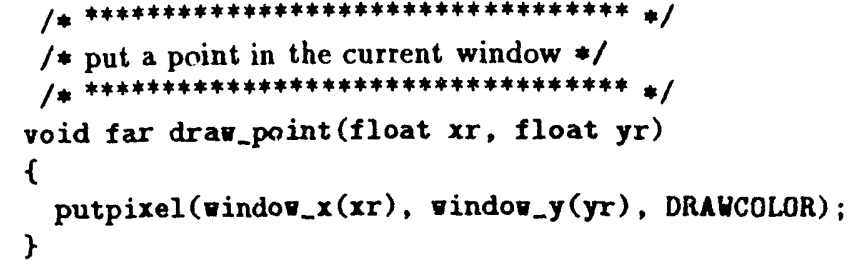

\section{F .10 PUT_CORD.C}

$1 * * * * * * * * * * * * * * * * * * * * * * * * * * * * * * * * * * * * * * * * * * * * * * * * * * * * * * * * * * * * * * * * * * * * * 1$

1* PUT_CORD.C *1

$1 * * 1$

/* This file contains part of a windowing graphics package written by */

/* Steven R. Brown and edited by Robert D. Hardy */

/* Sandia National Laboratories Dept. 5117. */

$1 * * 1$

1* comments configured for C2LATEX and $\operatorname{LAT}_{\mathrm{E}} \mathrm{X} * 1$

$/ * * * * * * * * * * * * * * * * * * * * * * * * * * * * * * * * * * * * * * * * * * * * * * * * * * * * * * * * * * * * * * * * * * * 1$

/* coordinate display routines for SRBGRAPH library $* 1$

*define GRAPH_LIB

/* provides the data definitions */

*include "srbgraph.h"

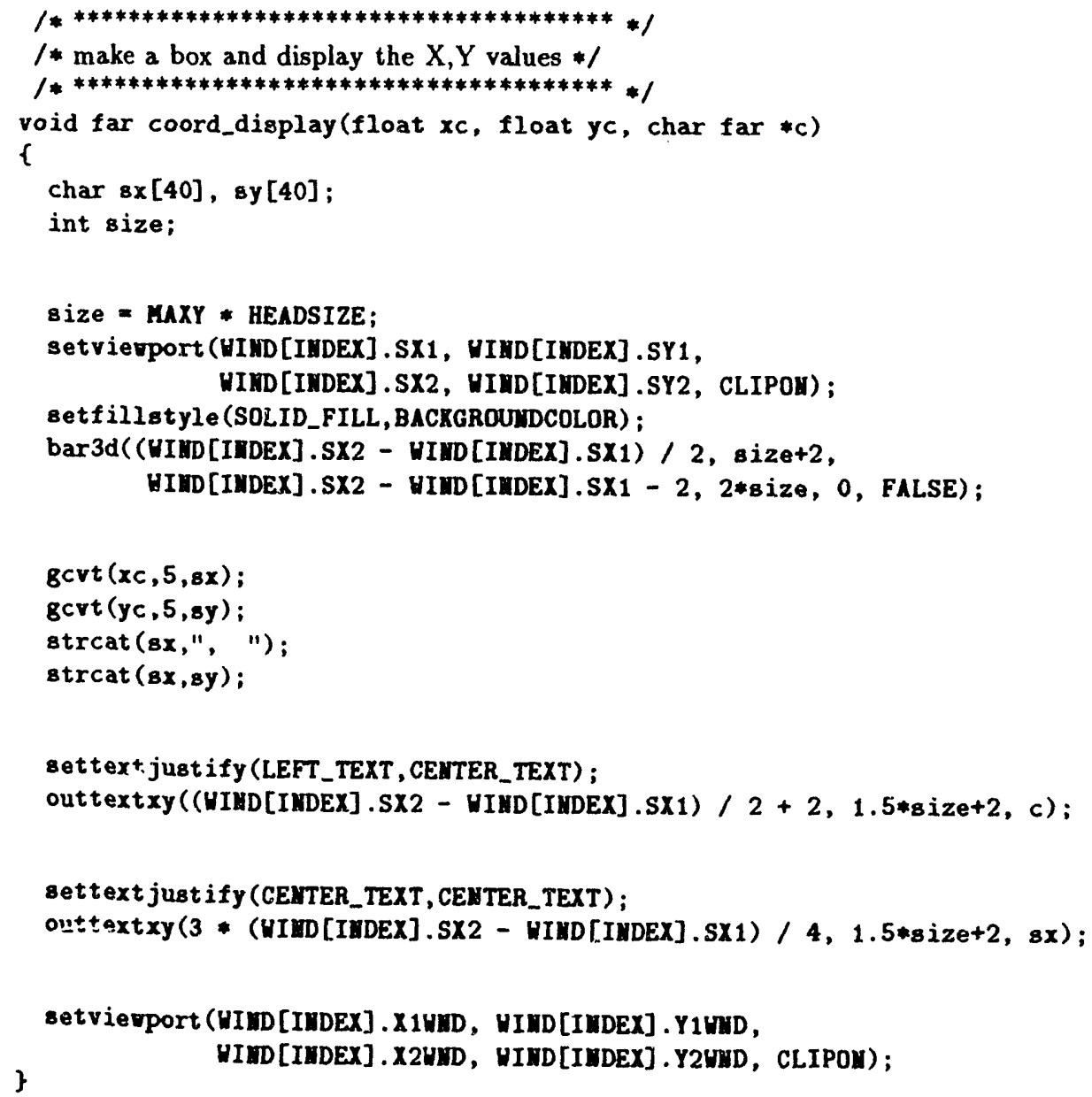




\section{F.11 CLR_CORD.C}

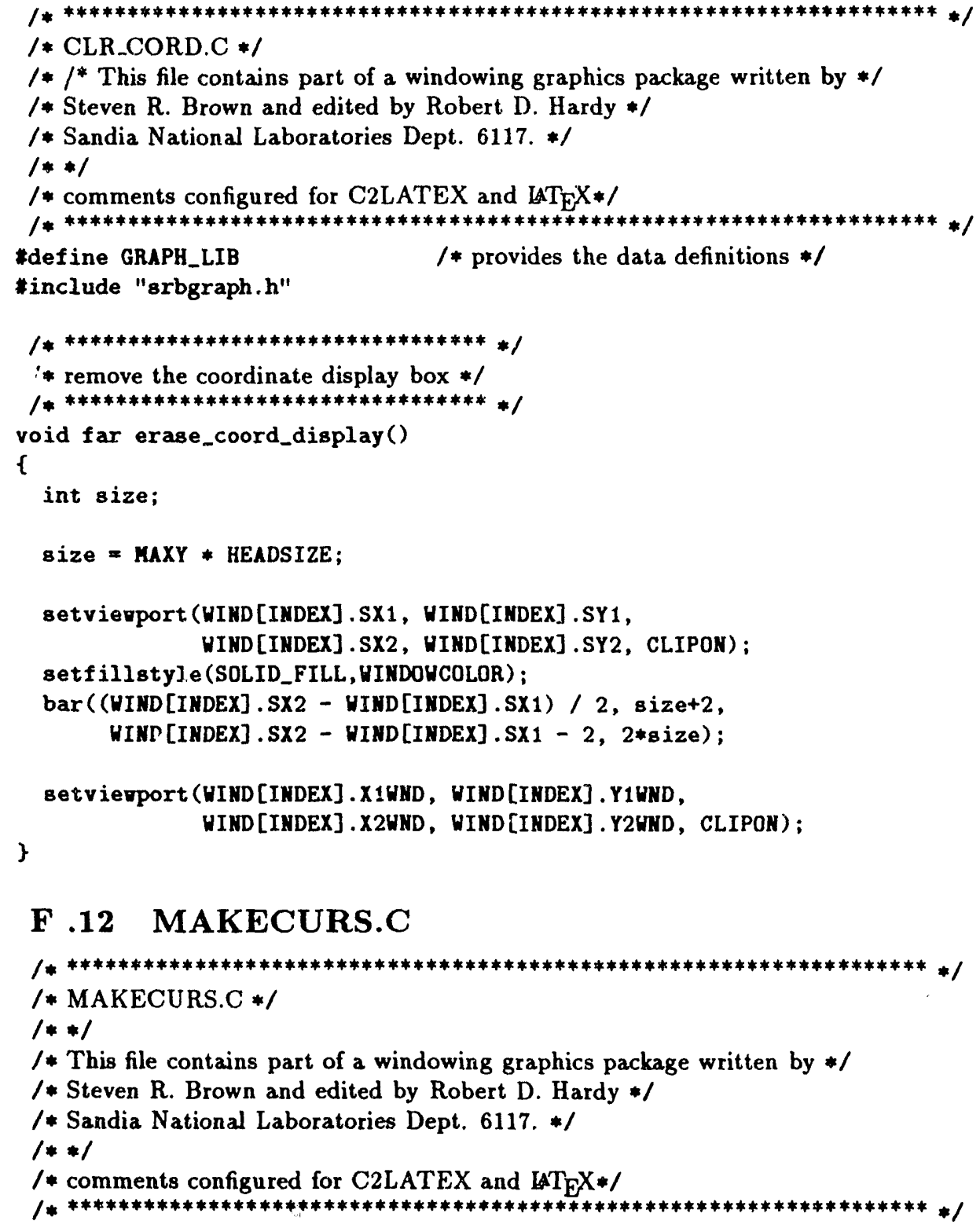

\section{F .12 MAKECURS.C}

$/ * * * * * * * * * * * * * * * * * * * * * * * * * * * * * * * * * * * * * * * * * * * * * * * * * * * * * * * * * * * * * * * * * * * * * /$

1* MAKECURS.C */

$1 * * 1$

/* This file contains part of a windowing graphics package written by */

/* Steven R. Brown and edited by Robert D. Hardy */

1* Sandia National Laboratories Dept. 6117. */

$1 * * 1$

1* comments configured for C2LATEX and $\mathrm{LT}_{\mathrm{E}} \mathrm{X} * 1$

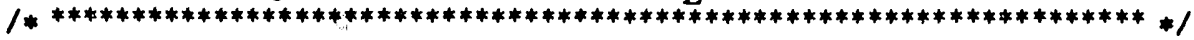

cursor routines for SRBGRAPH library

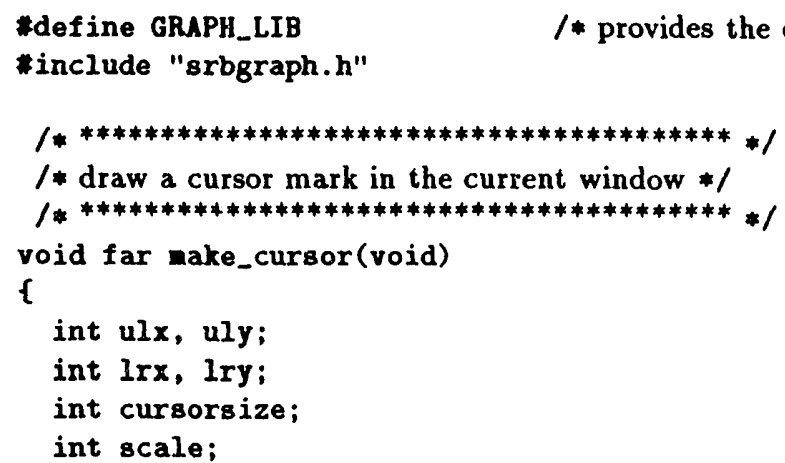




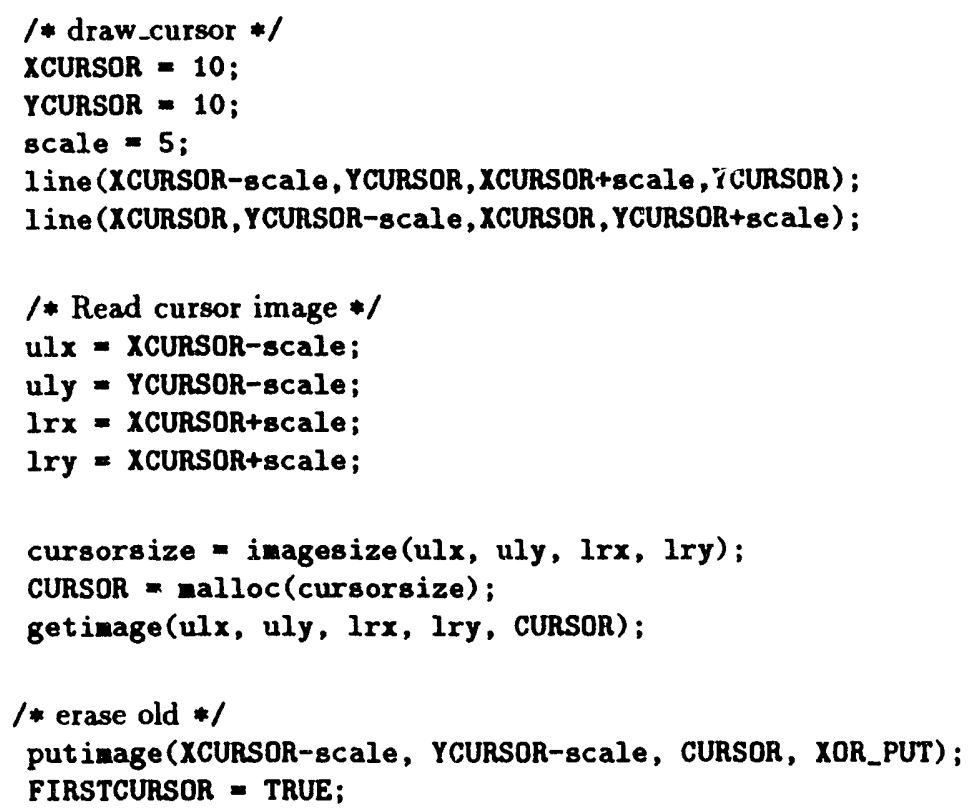

\section{F .13 PUT_CURS.C}

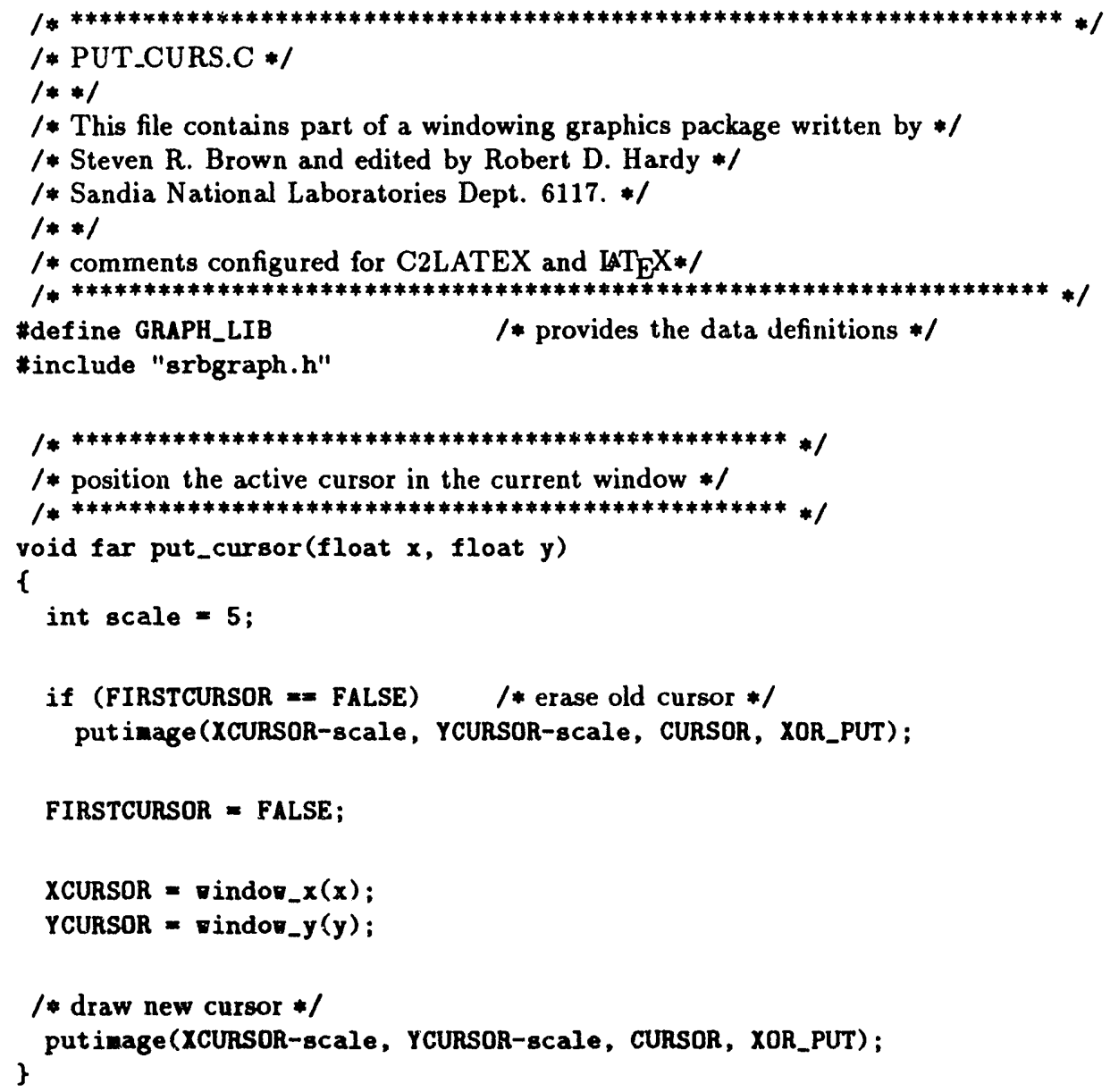




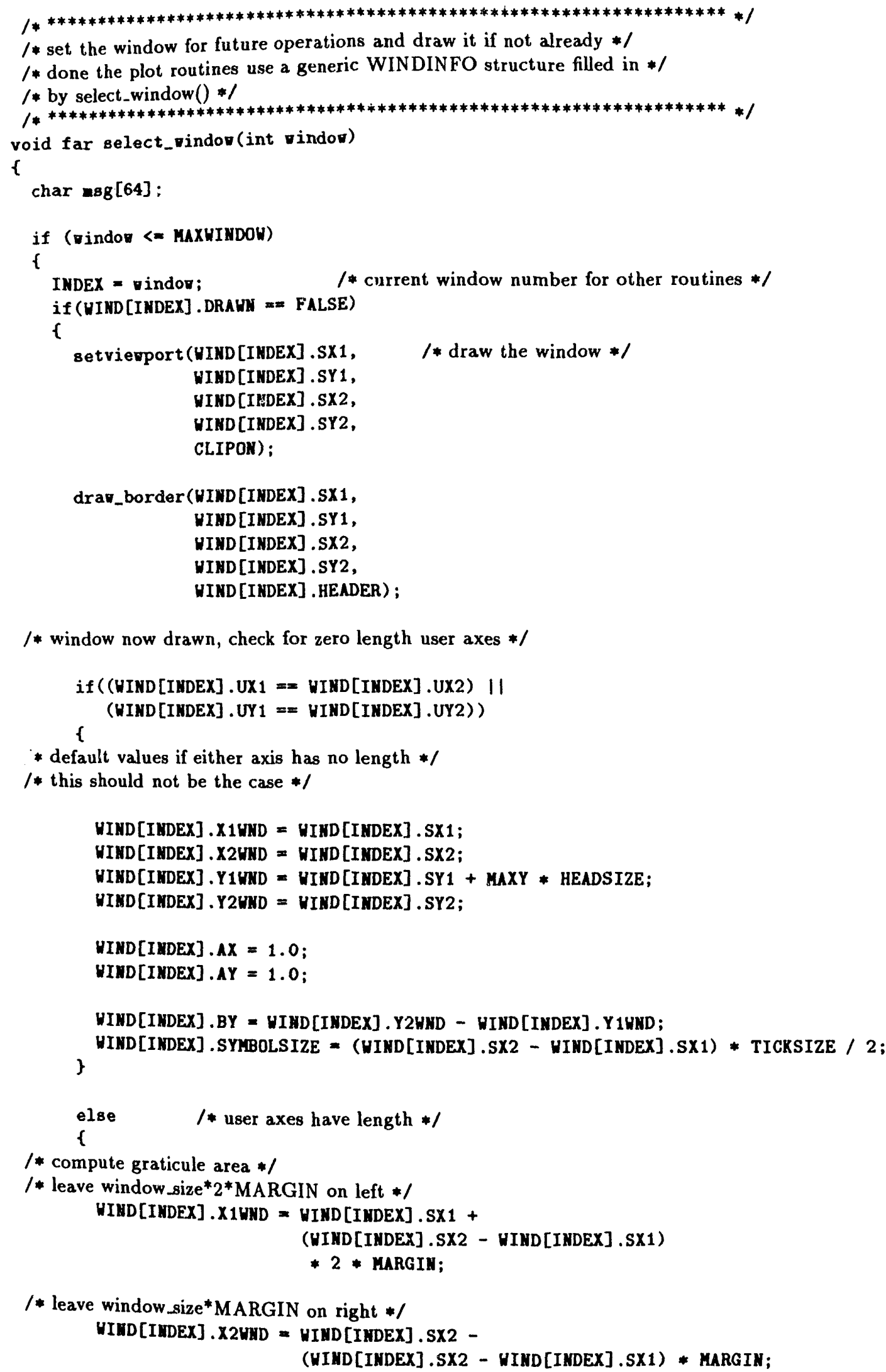

/* window now drawn, check for zero length user axes */

* default values if either axis has no length */

$1 *$ this should not be the case *I 


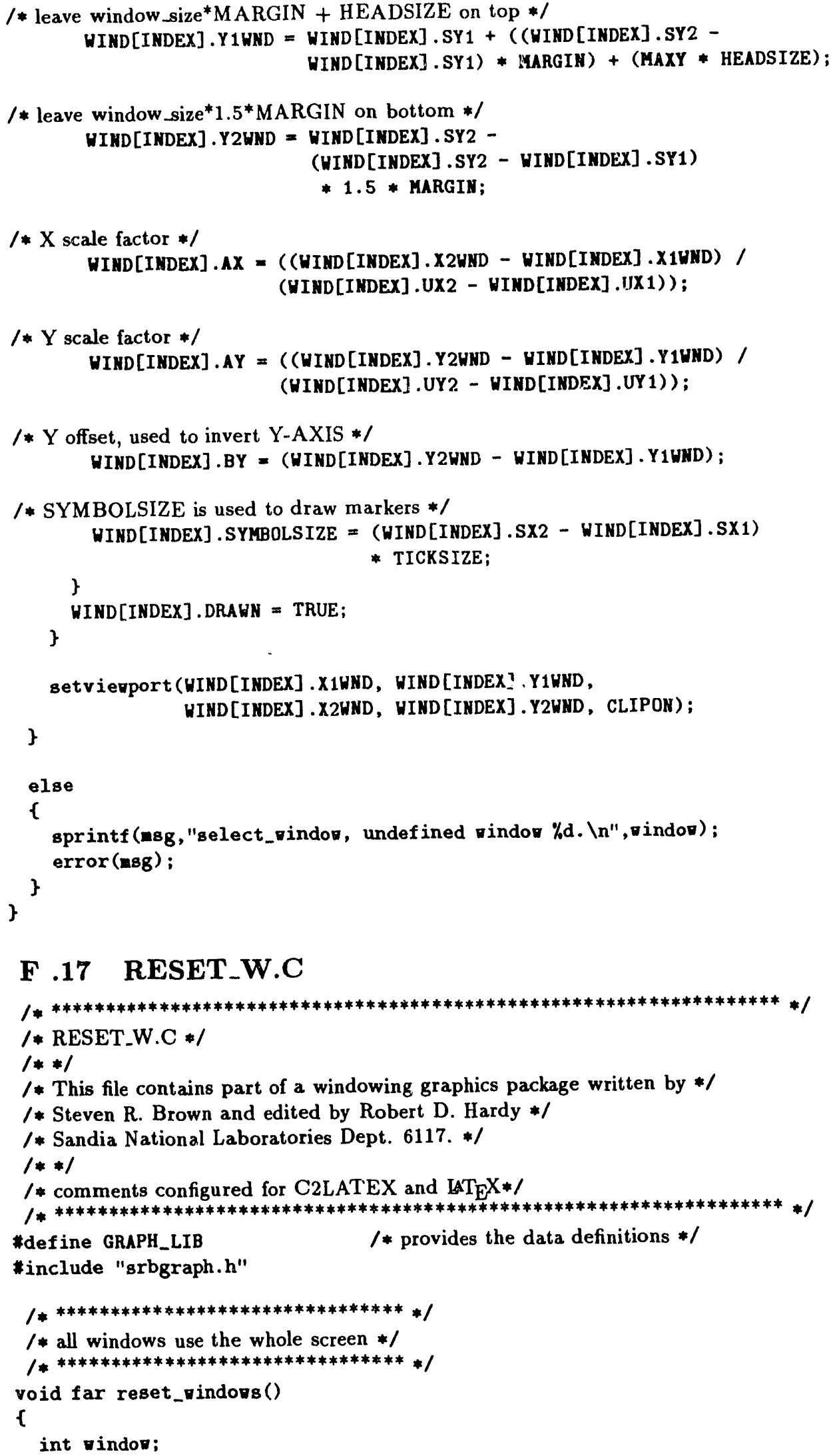

\section{F .17 RESET_W.C}

$/ * * * * * * * * * * * * * * * * * * * * * * * * * * * * * * * * * * * * * * * * * * * * * * * * * * * * * * * * * * * * * * * * * * * * * /$

/* RESET_W.C*I

$1 * * 1$

/* This file contains part of a windowing graphics package written by $*$ /

/* Steven R. Brown and edited by Robert D. Hardy */

/* Sandia National Laboratories Dept. 6117. */

$1 * * 1$

1* comments configured for C2LATEX and LAT $\mathrm{EX} * 1$

$/ * * * * * * * * * * * * * * * * * * * * * * * * * * * * * * * * * * * * * * * * * * * * * * * * * * * * * * * * * * * * * * * * * * * * 1$

*define GRAPH_LIB

$/ *$ provides the data definitions $* /$

\#include "srbgraph.h" 


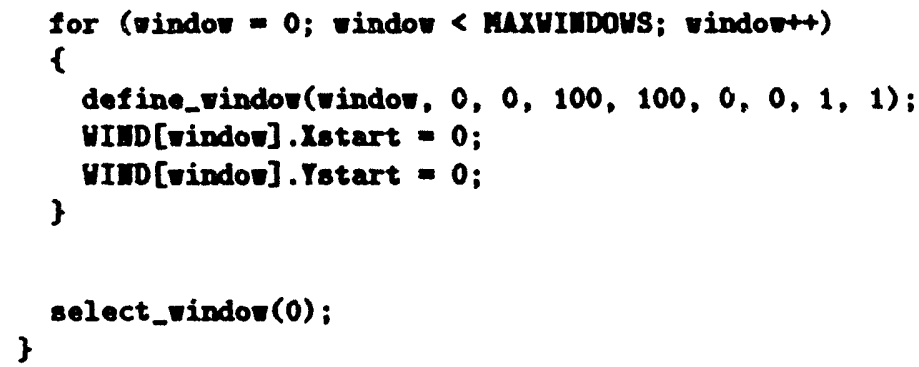

\section{F .18 ERASE_W.C}

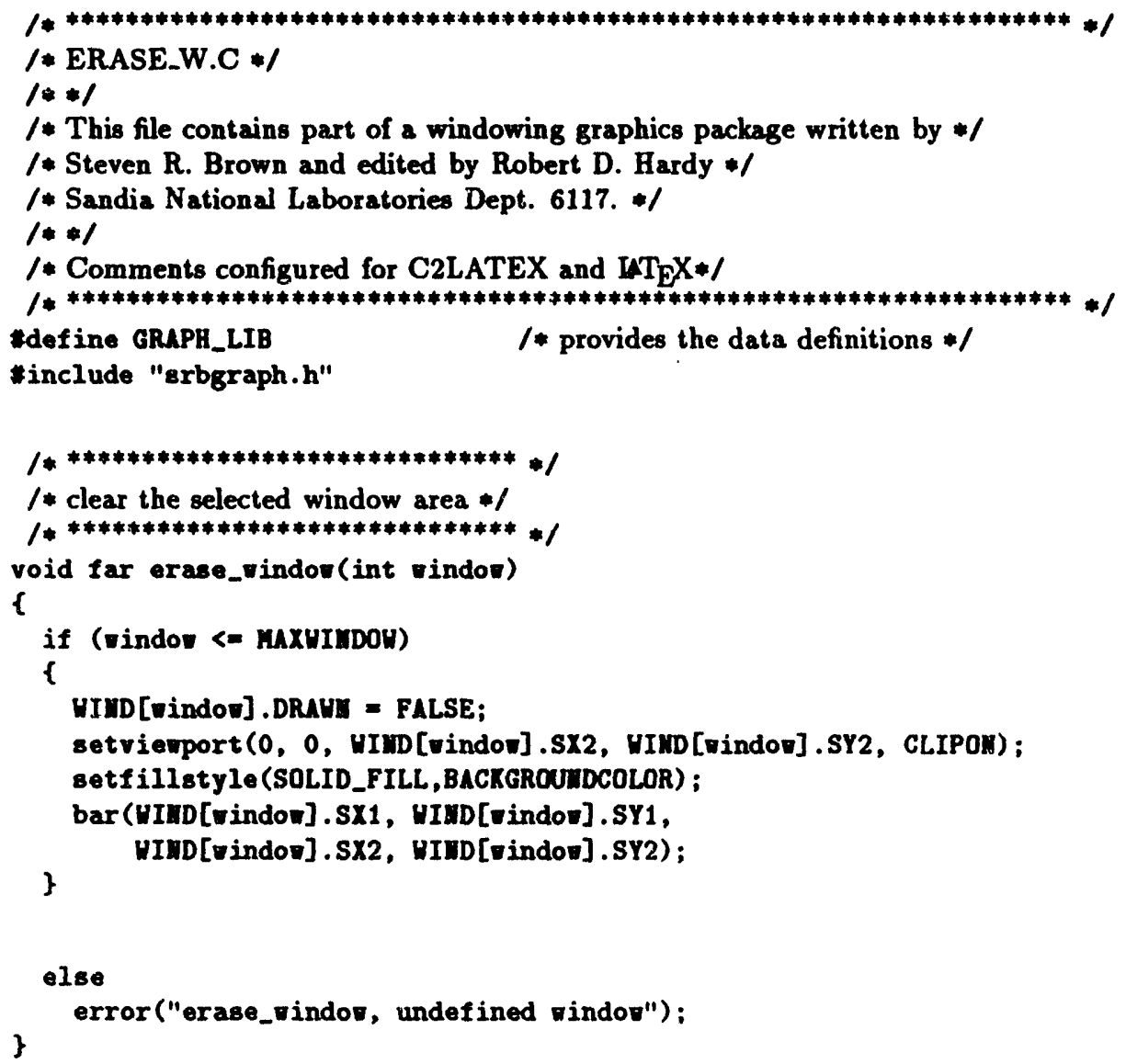

\section{F .19 ERROR.C}

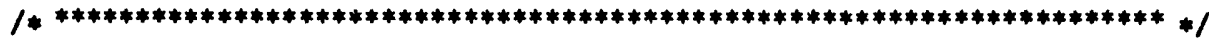

/* ERROR.C */

/* $/ *$ This file contains part of a windowing graphics package written by */

/* Steven R. Brown and edited by Robert D. Hardy */

1* Sandia National Laboratories Dept. 6117. */

$1 * * 1$

/* comments configured for C2LATEX and LTT $\mathrm{X} * /$

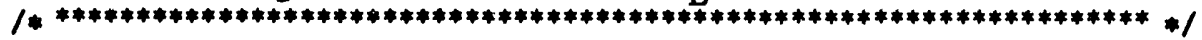

tefine GRAPB_LIB

/* provides the data definitions $* /$

*include "srbgraph.h" 


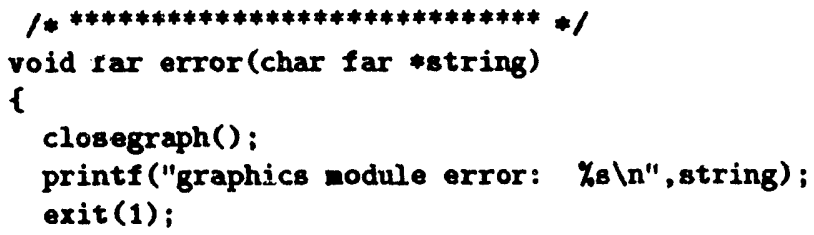

\section{F .20 SCALE.C}

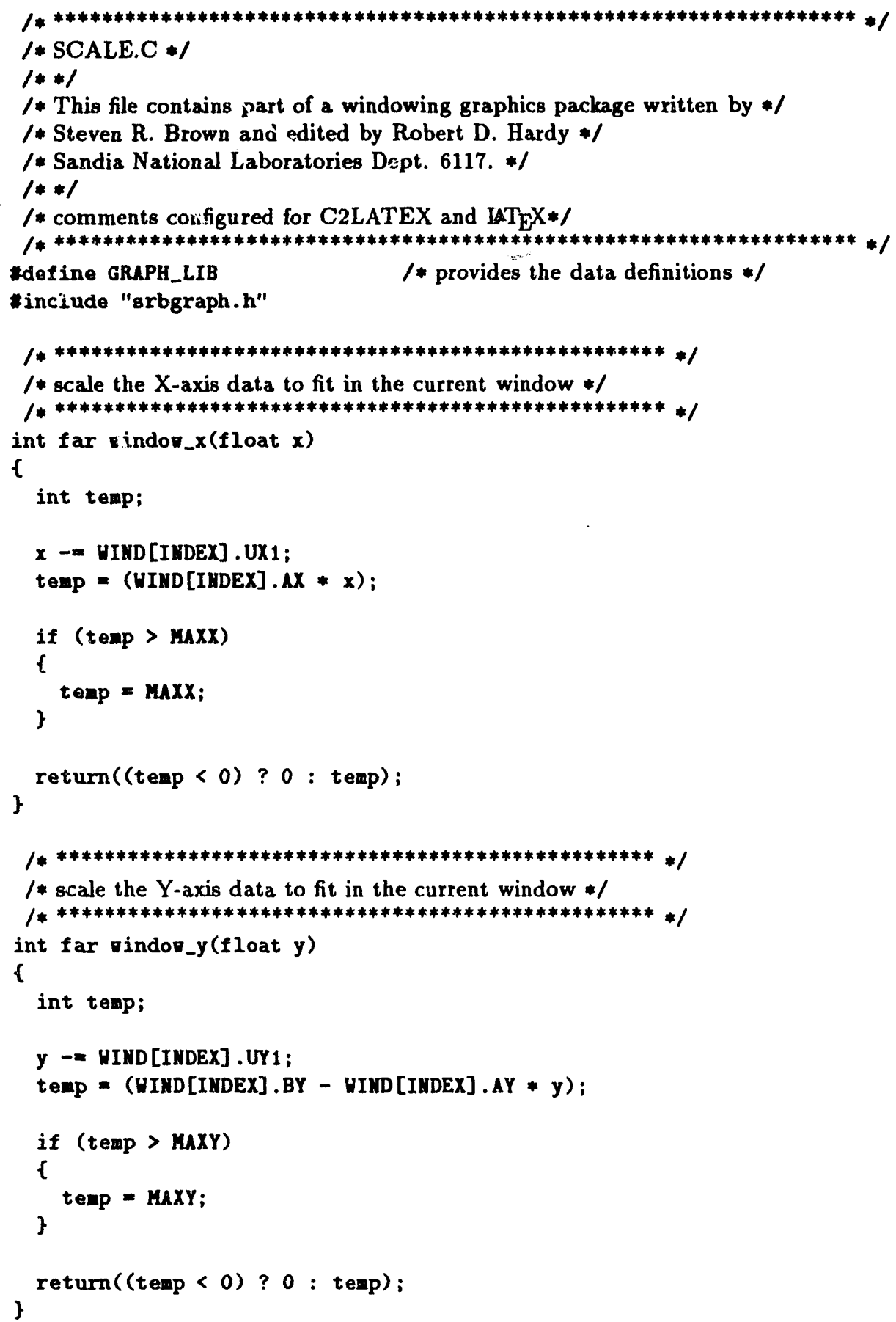




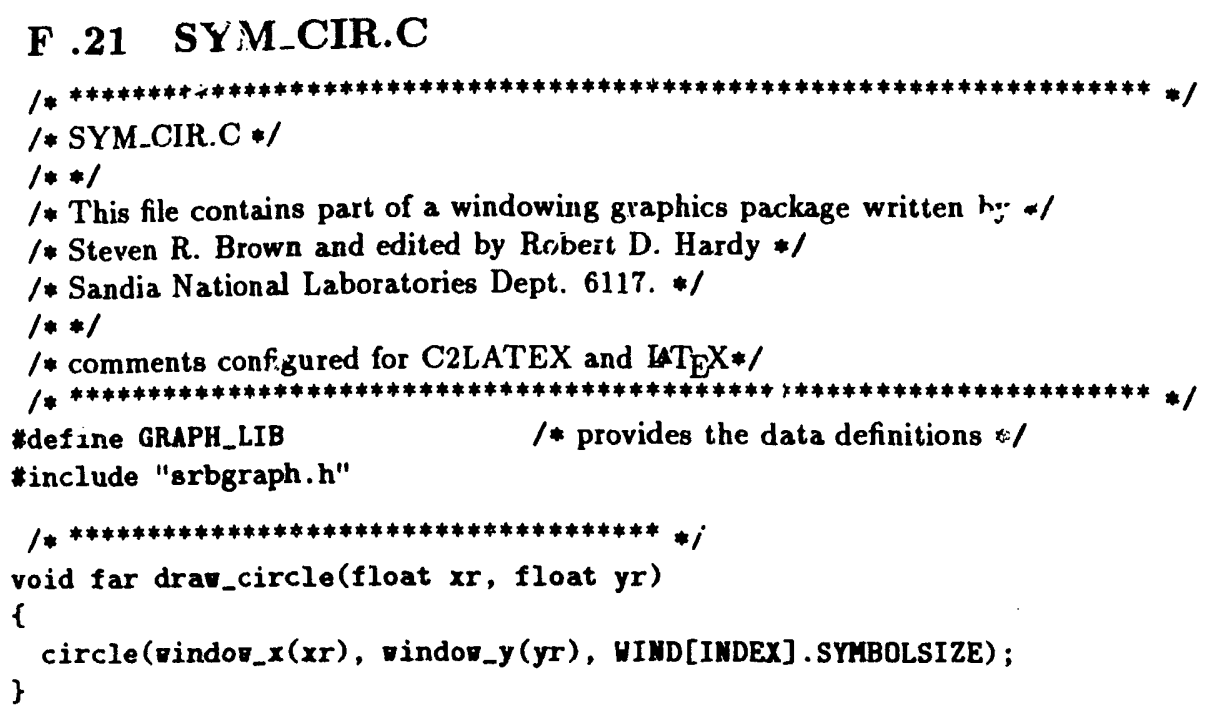

\section{F .22 SYM_CROS.C}

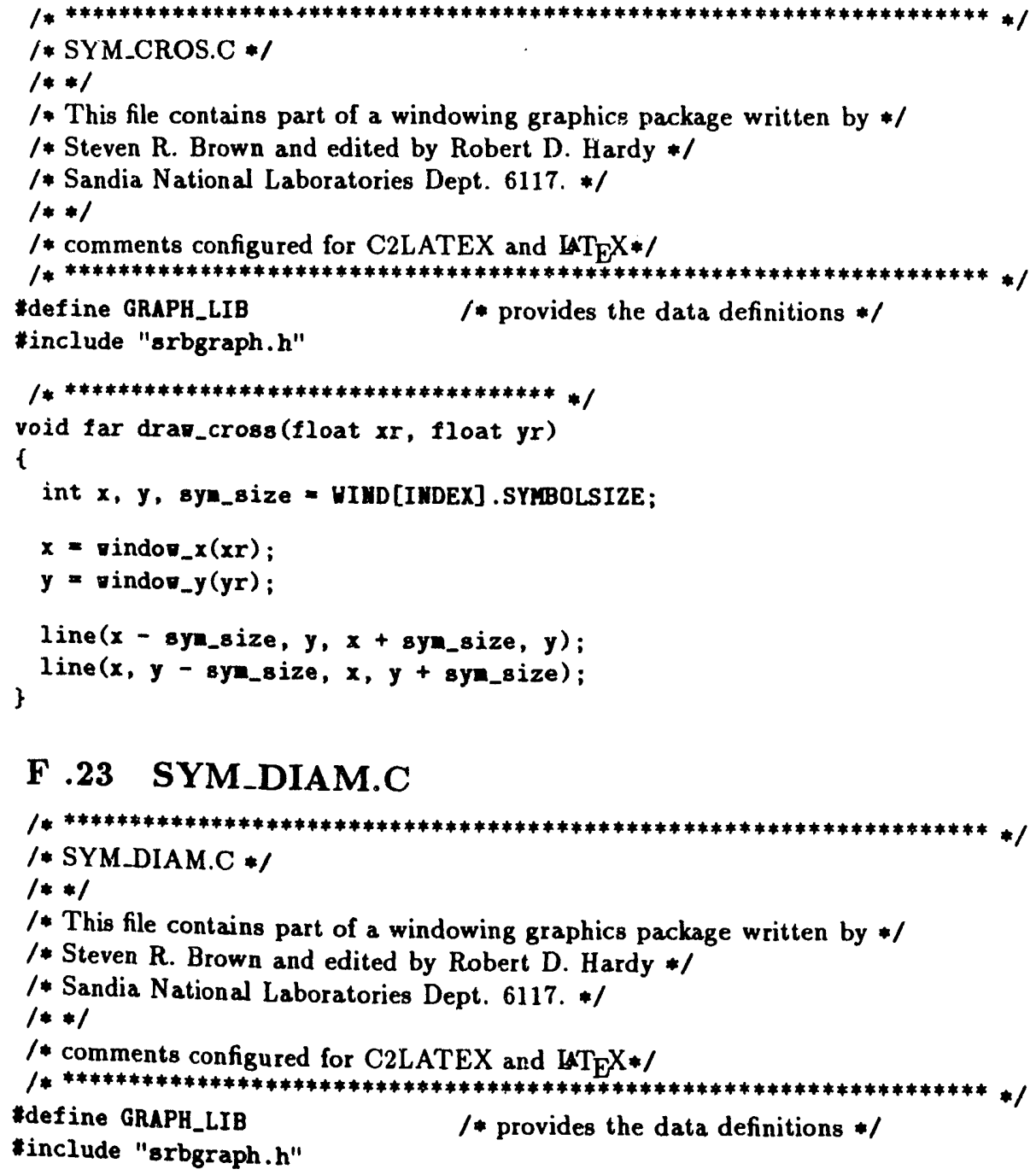

\section{F .23 SYM_DIAM.C}

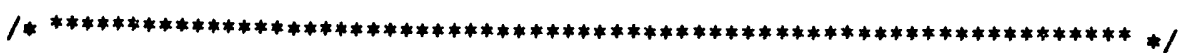
/* SYM_DIAM.C */

$1 * * 1$

1* This file contains part of a windowing graphics package written by */

/* Steven R. Brown and edited by Robert D. Hardy */

1* Sandia National Laboratories Dept. 6117. */

$1 * * 1$

1* comments configured for C2LATEX and $\mathrm{LT}_{\mathrm{E}} \mathrm{X} * 1$

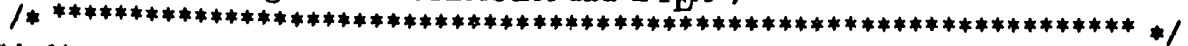

*define GRAPH_LIB

/* provides the data definitions $* /$

*include "srbgraph.h" 


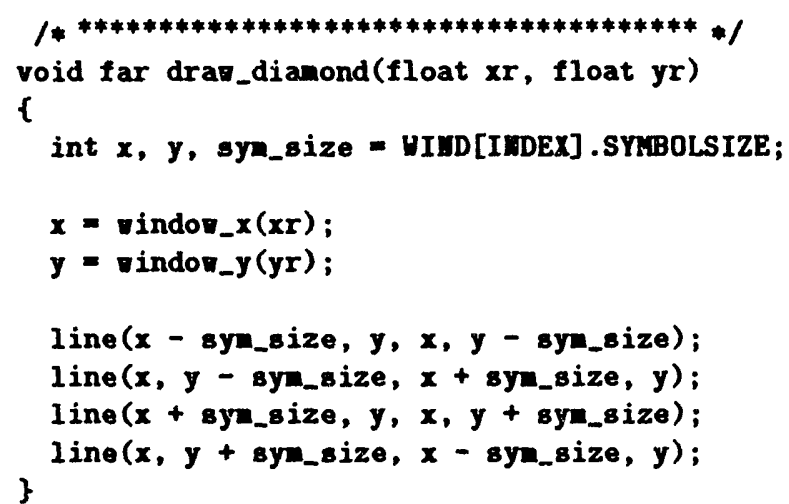

\section{F .24 SYM_SQR.C}

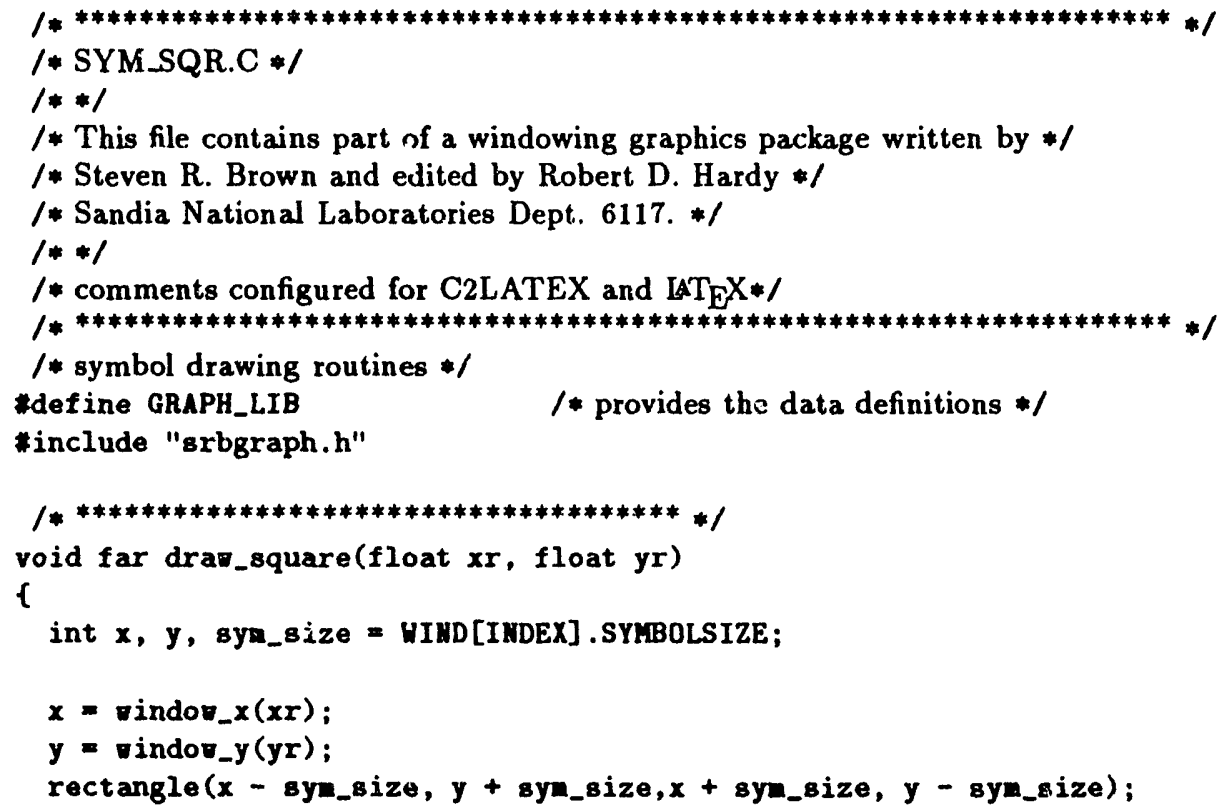

\section{F .25 SYM_STAR.C}

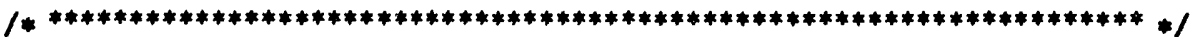
/* SYM.STAR.C */

$1 * * 1$

/ This file contains part of a windowing graphics package written by */

/* Steven R. Brown and edited by Robert D. Hardy */

/* Sandia National Laboratories Dept. 6117. */

$1 * * 1$

/* comments configured for C2LATEX and $\mathrm{LT}_{\mathrm{E}} \mathrm{X} * /$

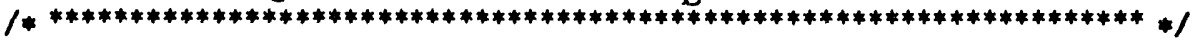

*define GRAPH_LIB

/* provides the data definitions $*$ /

*include "srbgraph.h"

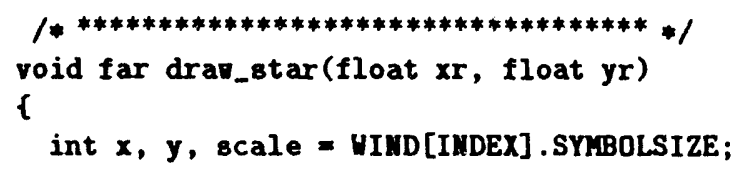




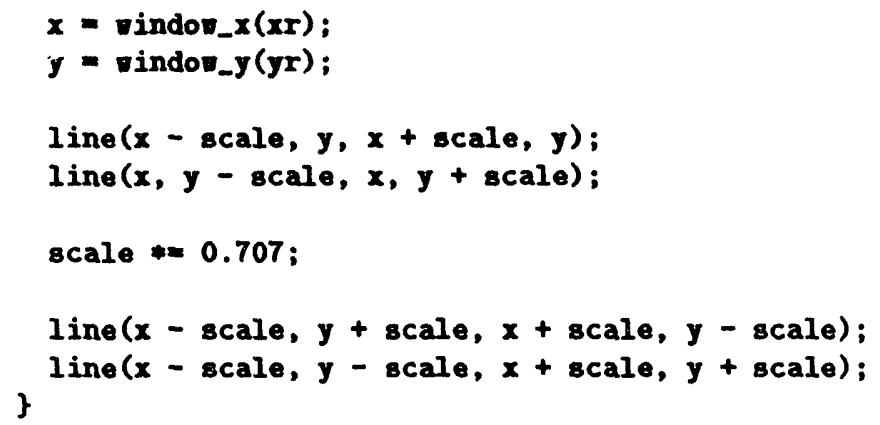

\section{F .26 SYM_TRI.C}

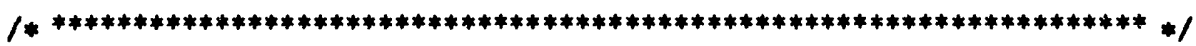
/*SYM-TRI.C *I

$1 * * 1$

1* This file contains part of a windowing graphics package written by */ /* Steven R. Brown and edited by Robert D. Hardy */

1* Sandia National Laboratories Dept. 6117. */

$1 * * 1$

/* comments configured for C2LATEX and LTTE $\mathrm{X} * /$

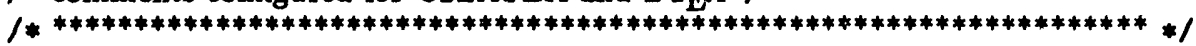

*define GRAPH_LIB

/* provides the data definitions */

*include "srbgraph.h"

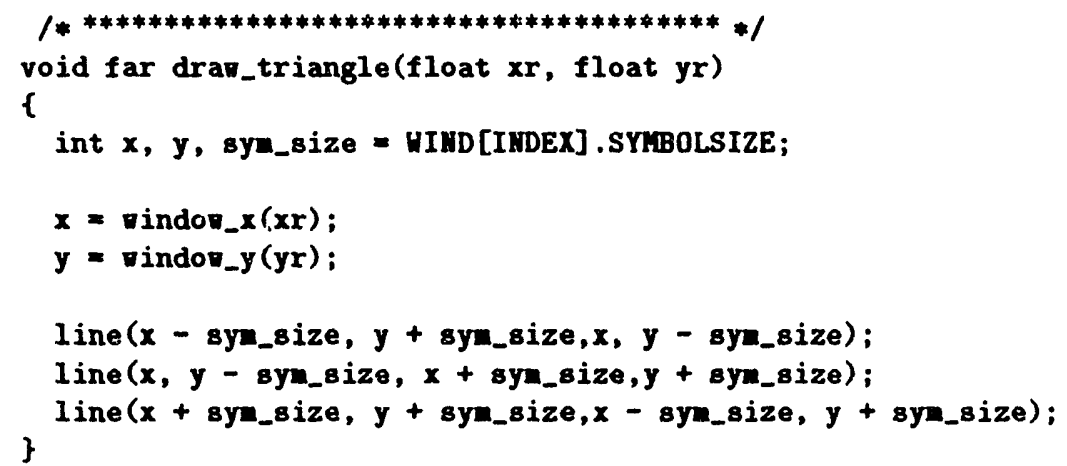

\section{F .27 SYM_X.C}

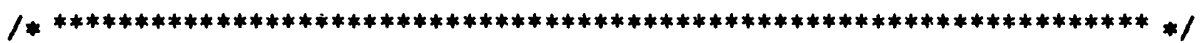
/* SYM.X.C *I

$1 * * 1$

/* This file contains part of a windowing graphics package written by */ /* Steven R. Brown and edited by Robert D. Hardy */

/* Sandia National Laboratories Dept. 6117. */

$1 * * 1$

/* comments configured for C2LATEX and LAT $\mathrm{EX} *$ /

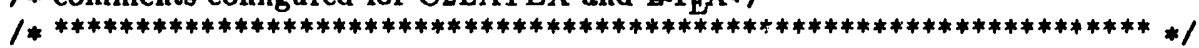

\#define GRAPH_LIB

\#include "srbgraph.h"

/* provides the data definitions */

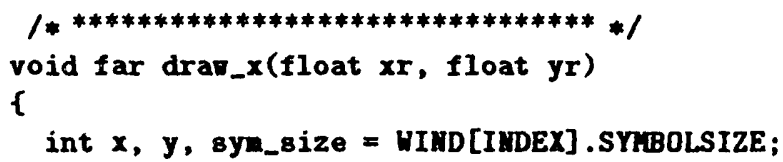


$x=$ vindo $\nabla_{-} x(x \mathbf{r})$;

$y=$ vindov_y $(y r)$;

line $\left(x-8 y m_{-} 8 i z e, y+8 y m_{-} 8 i z e, x+8 y m_{-} 8 i z e, y-8 y m_{-} s i z e\right) ;$

\}

line $\left(x-8 y m_{-} s i z e, y-8 y m_{-} s i z e, x+8 y m_{-} s i z e, y+s y n_{-} 8 i z e\right)$; 


\section{G BUILDTC.C}

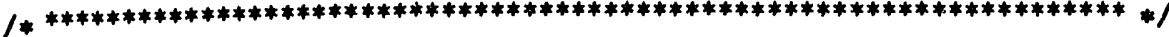

/* BUILDTC.C */

/* Program to build spline tables for thermocouple data in DATAVG. */

$1 * * 1$

/* Written by: *I

/* Robert D. Hardy */

/* Sandia National Laboratories */

/* Dept. 6117 */

/* April 17, $1992 * /$

$1 * * 1$

/* Comments configured for C2LATEX and $\mathrm{LT}_{\mathrm{E}} \mathrm{X} * 1$

$1 * * i$

$1 * * * * * * * * * * * * * * * * * * * * * * * * * * * * * * * * * * * * * * * * * * * * * * * * * * * * * * * * * * * * * * * * * * * * * 1$

1* The first line of a data set contains the thermocouple type $*$ /

/* letter followed by the number of data points to follow $(\mathrm{N}) . * /$

/* Input data is temperature, millivolts pairs separated by commas */

/* with one point per line. */

$1 * * 1$

1* This program computes coefficients and creates a file containing *j

$1 *$ the voltage, temperature and coefficient numbers with voltage */

/* scaled for board gain of 100 as done on the Keithley AIM7. The */

1* output consists of $\mathrm{N}$ sets of numbers preceded by a header line. */

1* The header gives the thermocouple type letter in upper case $*$ /

/* followed by the number of points in the data (N). Each data */

1* point occupies one line in the file. The line contains three */

/* columns of ASCII characters with comma separators. The columns */

1* are temperature, voltage and the coefficient numbers in that */

/* order. */

$1 * * 1$

/* The data for each type of thermocouple follows the same format */

/* with no particular order. This allows flexibility in */

$1 *$ configuration for a particular requirement. If few thermocouple */

$1 *$ types are used the tables may be small and therefore consume less */

1* memory. *1

$/ * * * * * * * * * * * * * * * * * * * * * * * * * * * * * * * * * * * * * * * * * * * * * * * * * * * * * * * * * * * * * * * * * * * * *)$

include <stdio.h>

\#include 〈stdlib.h〉

include 〈ctype.h>

\#include "spline.h"

roid get_temp(roid);

void put_temp(void);

/* arrays used for spline fitting to thermocouple data */

$1 *$ allow for 1000 points */

double $x a[1000]$;

/* Voltage */

double ya[1000];

/*Temperature $* /$

double y2a[1000];

$/ *$ Spline coefficient $* /$ 


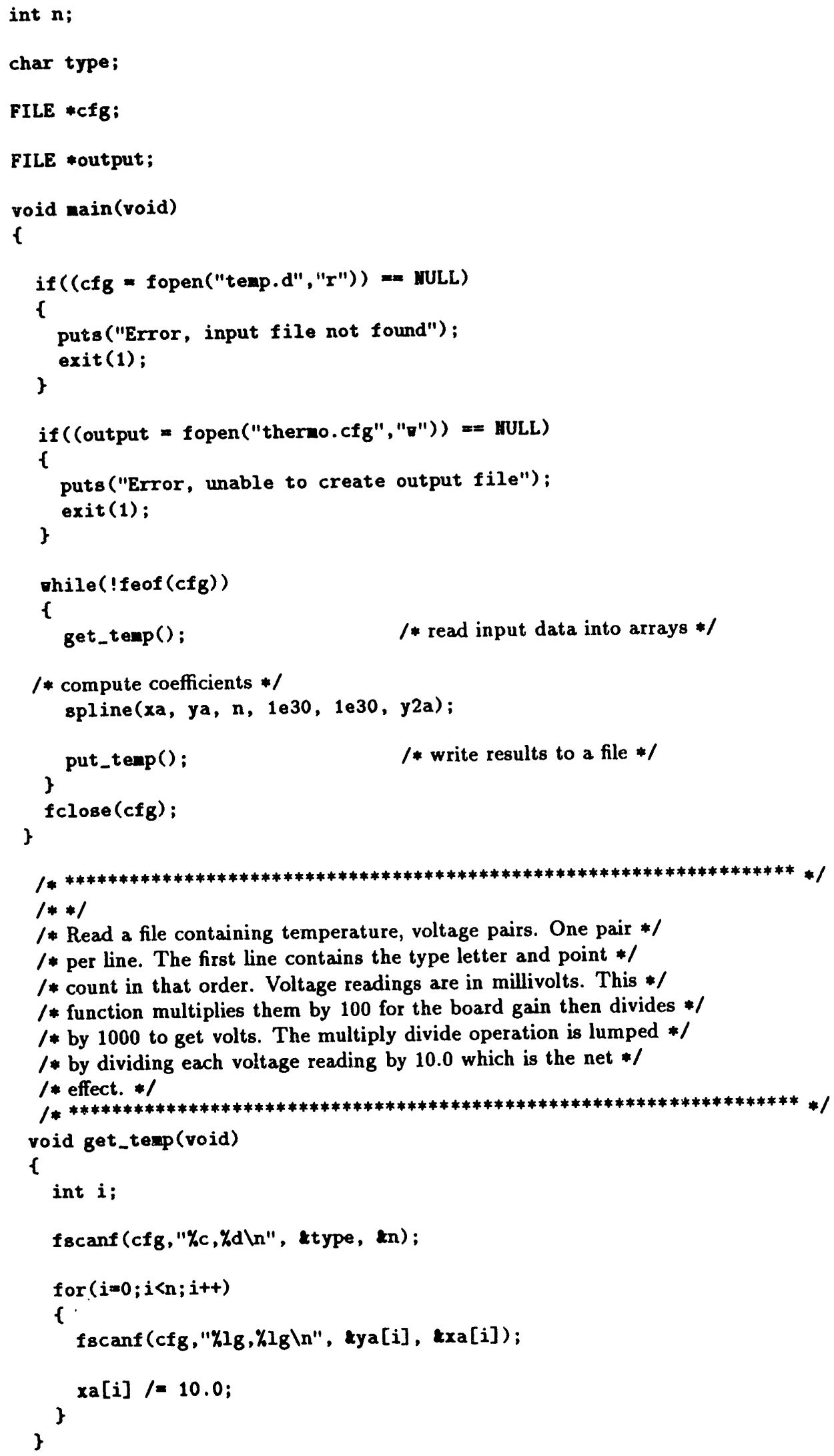




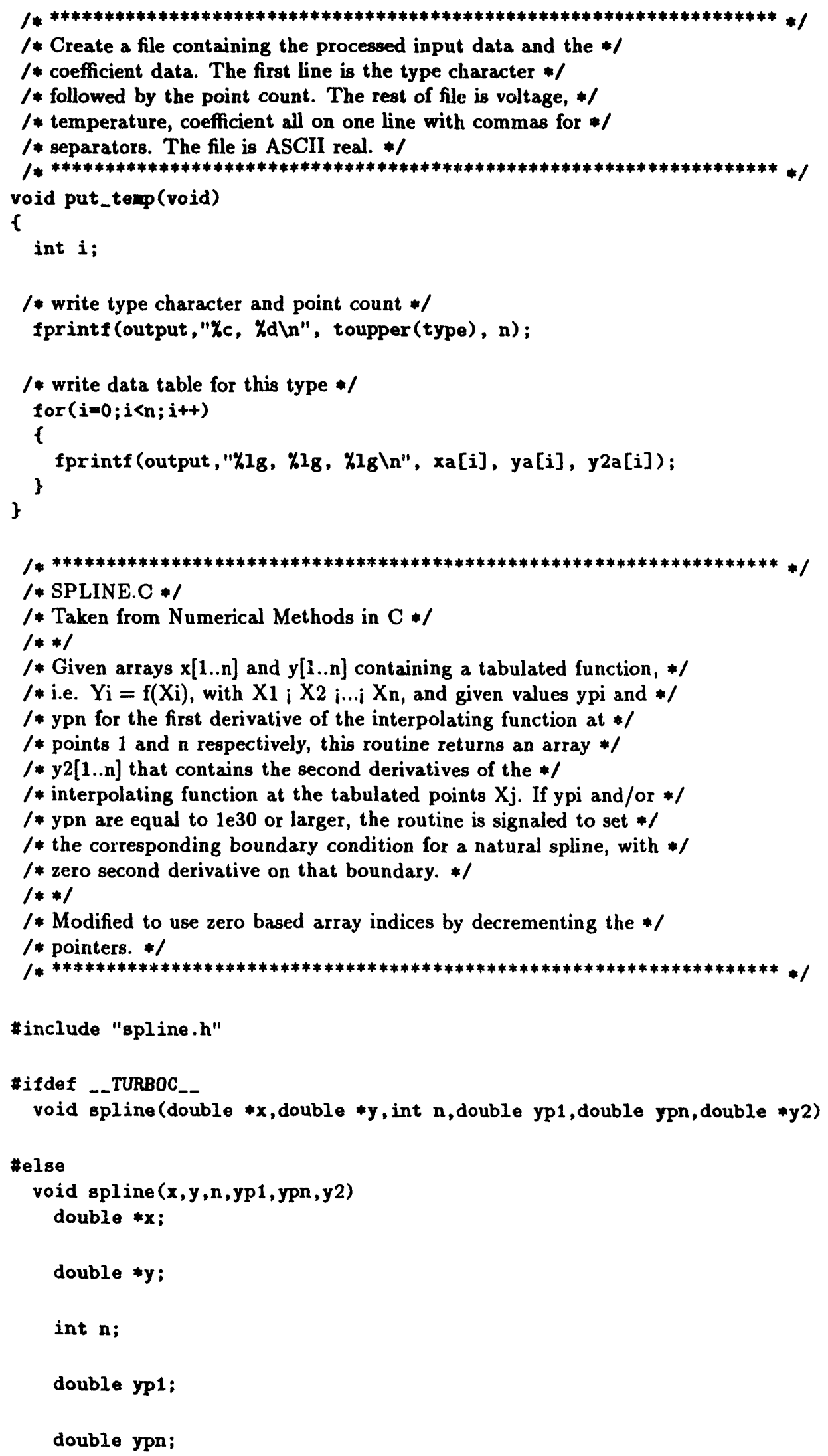




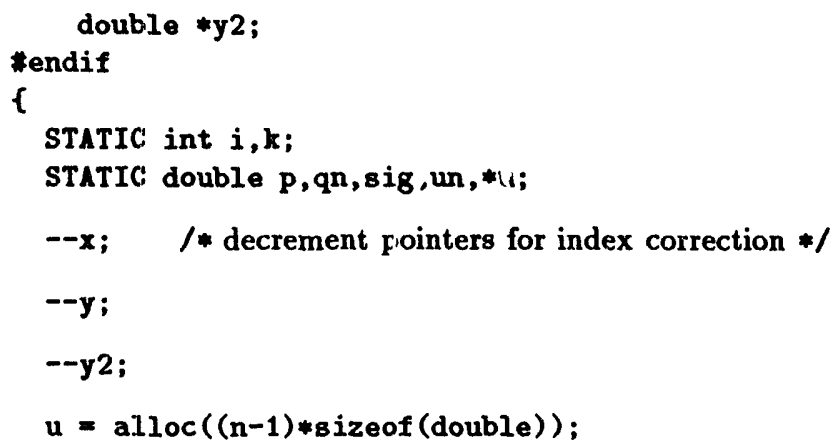

/* Set the lower boundary to be "natural" or to have a specified first derivative. */

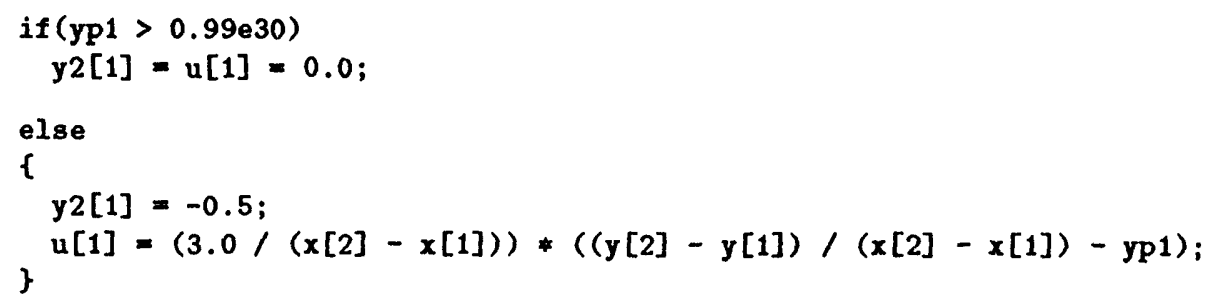

/* This is the decomposition loop of the tridiagonal algorithm. */

$/ * \mathrm{y} 2[]$ and $\mathrm{u}[\mathrm{are}$ used for temporary storage of the decomposed factors. */

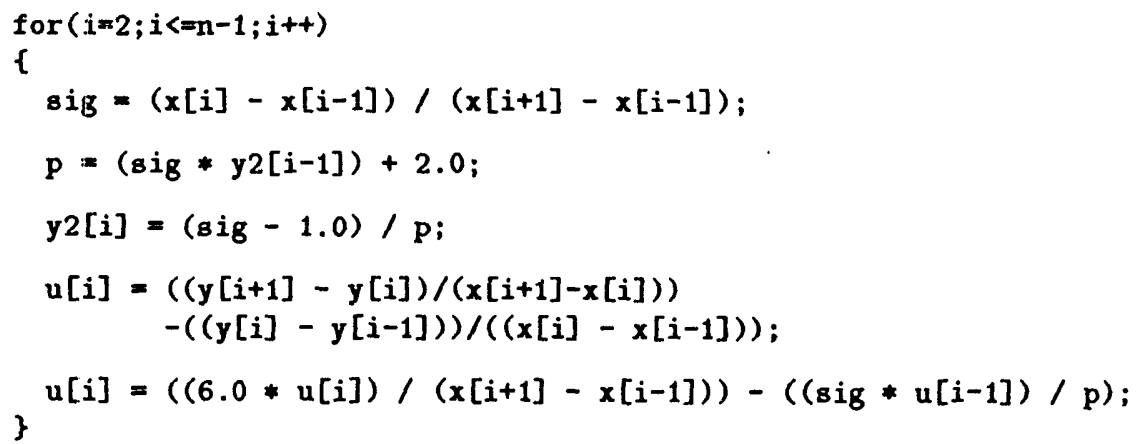

/*This is the back-substitution loop of the tridiagonal algorithm */ 


\section{Distribution}

Internal Distribution:

6100 R. W. Lynch

6111 J. L. Wise

6117 S. R. Brown

6117 L. W. Carlson

6117 D. J. Holcomb

6117 J. C. Lorenz

6117 W. A. Olsson

6117 D. S. Preece

6117 L. W. Teufel

6117 T. V. Tormey

6117 W. R. Wawersik

6117 D. H. Zeuch

6117 D. J. Zimmerer

6117 R. D. Hardy (10)

6117 Geomechanics Laboratory File (10)

7141 Technical Library ( 5)

7151 Technical Publications Department

7613-2 Document Processing for DOE/OSTI (10)

8523-2 Central Technical Files 

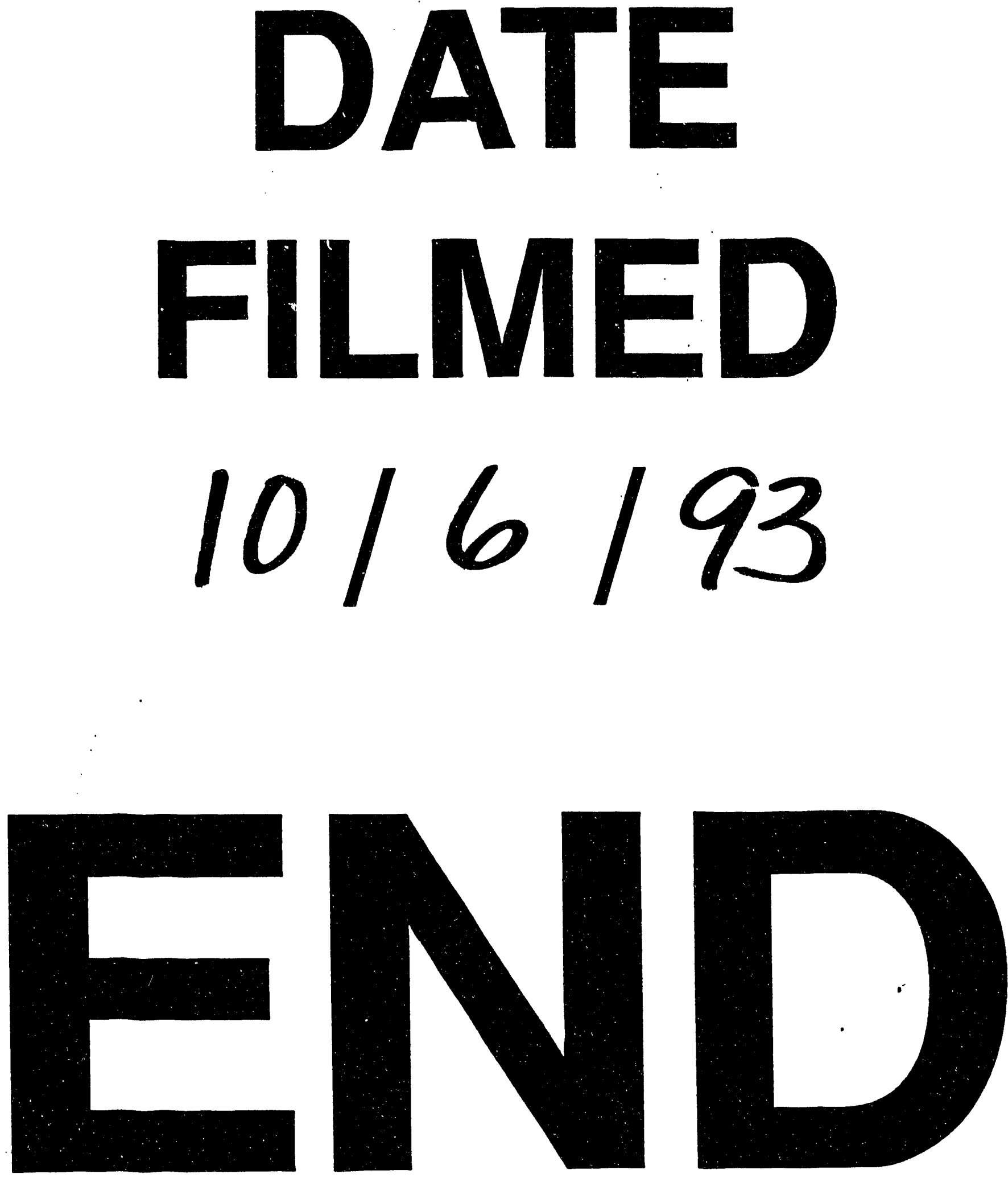
